\title{
Modes of Bars
}

\section{Contents}

$5.1 \quad$ Longitudinal Waves in Thin Bars ................................ 236

5.1 .1 Longitudinal Waves in Bulk Solids .............................. 240

5.1.2 The Quartz Crystal Microbalance ............................... 240

5.1.3 Bodine's "Sonic Hammer" ..................................... 242

5.2 Torsional Waves in Thin Bars ................................. 245

$5.3 \quad$ Flexural Waves in Thin Bars .................................... 246

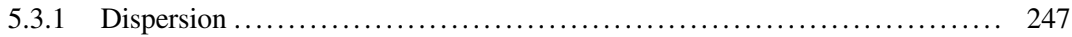

$5.3 .2 \quad$ Flexural Wave Functions .......................................... 249

$5.3 .3 \quad$ Flexural Standing Wave Frequencies .............................. 250

5.3.4 Flexural Standing Wave Mode Shapes .............................. 252

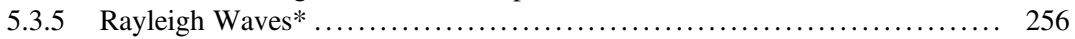

$5.4 \quad$ Resonant Determination of Elastic Moduli ........................ 256

5.4.1 Mode-Selective Electrodynamic Excitation and Detection ............... 258

5.4.2 Bar Sample Size and Preparation .................................... 259

5.4 .3 Measured Resonance Spectra ...................................... 260

5.4.4 Effective Length Correction for Transducer Mass ....................... 263

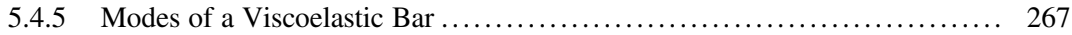

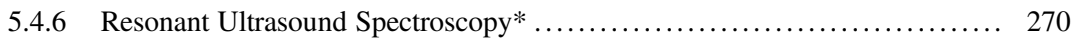

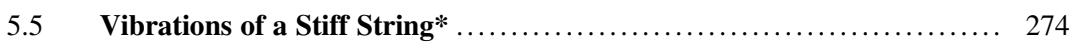

$5.6 \quad$ Harmonic Analysis ........................................... 277

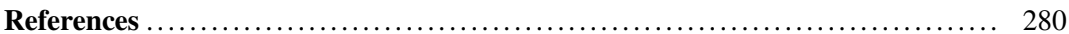

The perspectives and techniques that have been developed in the previous chapters will now be applied to calculation of wave propagation in solids. Their application to longitudinal and shear waves will be both familiar and simple. What you will find to be even more satisfying is the success of those same techniques for finding solutions for waves in a system that does not obey the wave equation and whose solutions are not functions of $x \pm c t$.

This chapter is focused mainly on waves in bars that are "thin," meaning that the square root of their cross-sectional area is much less than their length, $\sqrt{S} \ll L$. The frequencies of the normal modes of such thin bars will be used to determine the bars' elastic constants to high precision. The results for thin bars will also lead to the understanding of waves in samples with dimensions much greater than the 
wavelength of the sound. Examples of short wavelength propagation in "bulk" samples range from ultrasonic waves in crystals and for biomedical diagnosis and therapy to seismic waves that penetrate our Earth and the Sun.

Before initiating these next investigations, it may be worthwhile to review the sequence of steps we have taken thus far to calculate the behavior of harmonic oscillators and waves on strings:

- Determine the relationship that governs forces and displacements in our system of interest (e.g., Hooke's law, the equation of state, or the "constitutive equation").

- Examine the behavior of a differential element and determine the net forces (or net torques) acting on that element.

- Assume a sufficiently small displacement from equilibrium (i.e., "linear" behavior) and apply a Taylor series expansion to evaluate the net forces (or net torques) acting on the differential element.

- Use Newton's Second Law of Motion to calculate the acceleration of the differential element.

- Ignore any contributions to the equation relating accelerations to restoring forces that involve second-order quantities, such as products of two or more first-order deviations from equilibrium, like displacements or velocities, since such quadratic products are small compared to the linear contributions.

- Obtain a wave propagation speed.

- Apply the "machinery" to extract solutions under specific circumstances (e.g., harmonic analysis, superposition, boundary conditions, initial conditions, Fourier's theorem, mechanical impedance at steady state).

\subsection{Longitudinal Waves in Thin Bars}

There are three independent types of waves that can be excited in thin bars of solid materials at frequencies that are sufficiently low that the wavelengths of these waves are much greater than the cross-sectional dimensions (i.e., $\lambda \gg S^{1 / 2}$ ): (i) longitudinal waves of compression and expansion, (ii) torsional waves that produce twisting, and (iii) flexural waves that cause the bar to bend. Having examined the elastic moduli for isotropic solids, it should be clear that for longitudinal waves in thin bars, it is Young's modulus that quantifies the relationship between the longitudinal stresses and strains for a solid whose sides are unconstrained. Thus, the edges are free to "bulge" when compressed and to "neck down" when expanded. Assuming a bar of rectangular cross-section, as shown in Fig. 5.1, then $(\Delta w / w)=(\Delta h / h)=-\nu(\Delta \xi / d x)$.

The longitudinal strain, $\varepsilon_{x x}$, will be the ratio of the change in the length of the differential element from its original undisturbed length, $d x$, to its expanded length, $\xi(x+d x)-\xi(x)$, divided by its original length, $d x$. As before, a Taylor series expansion about $\xi(x)$ will be used to simplify the expression for the longitudinal strain.

$$
\varepsilon_{x x}=\frac{\xi(x+d x)-\xi(x)}{d x} \cong \frac{\left(\frac{\partial \xi}{\partial x}\right) d x}{d x}=\left(\frac{\partial \xi}{\partial x}\right)
$$

Using Eq. (4.1), longitudinal stress, $\sigma_{x x}$, will be related to that longitudinal strain, $\varepsilon_{x x}$, by Young's modulus, $E$, for the bar's material, allowing $S_{x}(x)$ to be the cross-sectional area of the bar as a function of position along the bar. 


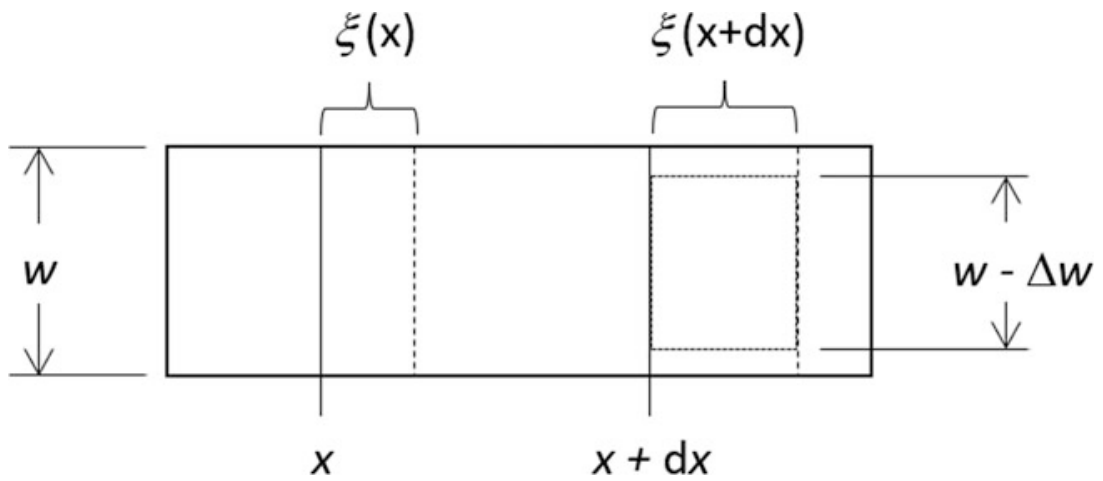

Fig. 5.1 Coordinate system for the displacement from equilibrium, $\xi(x)$, caused by a longitudinal wave passing through a thin bar. At the moment shown, the differential element of the bar has expanded, so its width (and height, not shown) will decrease in accordance with Poisson's ratio

$$
\sigma_{x x}=\frac{F_{x}(x)}{S_{x}(x)}=-E(x) \frac{\partial \xi(x)}{\partial x} \Rightarrow F_{x}=-E S_{x} \frac{\partial \xi}{\partial x}
$$

In the right-hand version, we have assumed that neither $E$ nor $S_{x}$ is a function of position, although the dependence of $S_{x}$ on strain, $\varepsilon_{x x}$, if $\nu \neq 0$, will be given in Eq. (5.4).

To determine the net longitudinal force, $d F_{x}$, acting on the differential element of length, $d x$, we expand Eq. (5.2) in a Taylor series about $F_{x}(x)$ and assume that Young's modulus is a constant.

$$
d F_{x}=F_{x}(x)-F_{x}(x+d x) \cong \frac{\partial F_{x}(x)}{\partial x} d x=E \frac{\partial}{\partial x}\left(S_{x} \frac{\partial \xi}{\partial x}\right) d x
$$

If the cross-sectional area of the bar is also a constant, it too can be taken outside of the derivative. Having introduced Poisson's ratio in Eq. (4.2), we know that the cross-sectional area will not remain constant. Again, assume a bar of rectangular cross-section, so $S=w h$, and then use logarithmic differentiation to calculate the relative change in cross-section.

$$
\frac{\delta S_{x}}{S_{x}}=\frac{\delta w}{w}+\frac{\delta h}{h}=-2 \nu \varepsilon_{x x}=-2 \nu \frac{d \xi}{d x}
$$

Substitution of Eq. (5.4) into Eq. (5.3) provides an expression for the net longitudinal force, $d F_{x}$, acting on the differential element.

$$
d F_{x}=E \frac{\partial}{\partial x}\left[S_{x}\left(1-2 \nu \frac{\partial \xi}{\partial x}\right) \frac{\partial \xi}{\partial x}\right] d x \cong E S_{x} \frac{\partial^{2} \xi}{\partial x^{2}} d x
$$

Expanding the term in square brackets, we see that the correction to the cross-sectional area, due to Poisson's ratio, is second order in the strain, thus can be neglected in a first-order (linear) analysis. Since we are making a linear approximation, the strains are assumed to be small, $\partial \xi / \partial x=\varepsilon_{x x} \ll 1$, so we can ignore the Poisson term because it is proportional to $\varepsilon_{x x}{ }^{2} \ll \varepsilon_{x x}$.

The net longitudinal force, $d F_{x}$, is equal to the acceleration of the mass of the differential element, $\rho S_{x} d x$, where $\rho$ is the mass density $\left[\mathrm{kg} / \mathrm{m}^{3}\right]$ of the bar's material at $x$. 


$$
\rho S_{x} d x \frac{\partial^{2} \xi}{\partial t^{2}}=E S_{x} \frac{\partial^{2} \xi}{\partial x^{2}} d x \quad \Rightarrow \quad \frac{\partial^{2} \xi}{\partial t^{2}}-\frac{E}{\rho} \frac{\partial^{2} \xi}{\partial x^{2}}=0
$$

We know that the solution to this wave equation will be the same superposition of a left- and rightgoing traveling wave as appeared in Eq. (3.18).

$$
\xi(x, t)=A \mathrm{e}^{j(\omega t-k x)}+B e^{j(\omega t+k x)}
$$

Equation (5.6) is a wave equation that reveals a propagation speed (phase speed) for longitudinal waves in thin bars, $c_{B}$.

$$
c_{B}=\frac{\omega}{k}=f \lambda=\sqrt{\frac{E}{\rho}}
$$

This speed of longitudinal wave propagation depends upon the ratio of the material's modulus to its mass density. It should also be noted that neither the cross-sectional area of the bar, $S_{x}$, nor the shape of that cross-section influences $c_{B}$, as long as the wavelength, $\lambda$, is much greater than the width, height, or diameter of the bar: $\lambda=c_{B} / f \ll \sqrt{S_{x}}$. Denser and softer metals, like lead, will have slower propagation speeds, while stiffer, less dense metals, like beryllium, will have faster speeds. For pure lead, $c_{B} \cong$ $1200 \mathrm{~m} / \mathrm{s}$ and for beryllium, $c_{B} \cong 12,870 \mathrm{~m} / \mathrm{s}$.

Using our prior experience with standing waves on strings of finite length (see Sect. 3.3.1), the process of imposing ideal boundary conditions should be familiar. Experimentally, the easiest boundary condition to apply to a thin bar of length, $L$, to be excited in its longitudinal mode, is that the displacements of both ends are unconstrained or "free." If there are no forces on the end at $x=0$ or the end at $x=L$, then Eq. (5.2) can be used to express these "free" boundary conditions.

$$
F_{x}=-E S_{x}\left(\frac{\partial \xi}{\partial x}\right)_{x=0}=-E S_{x}\left(\frac{\partial \xi}{\partial x}\right)_{x=L}=0
$$

The derivative of Eq. (5.7) with respect to $x$ is simplified by our choice of exponential functions of space and time.

$$
F_{x}(x, t)=-E S_{x}\left(\frac{\partial \xi}{\partial x}\right)=-j k E S_{x}\left[B e^{j(\omega t+k x)}-A \mathrm{e}^{j(\omega t-k x)}\right]
$$

The force at $x=0$ can be evaluated by setting Eq. (5.10) to zero. This boundary condition can be satisfied by the superposition of traveling waves in Eq. (5.7) if $A=B$.

$$
\begin{aligned}
\xi(x, t) & =\mathfrak{R} e\left[B\left(e^{j(\omega t+k x)}+\mathrm{e}^{j(\omega t-k x)}\right)\right] \\
& =\mathfrak{R} e\left[B e^{j \omega t}\left(\mathrm{e}^{j k x}+e^{-j k x}\right)\right]=\mathfrak{R} e\left[\widehat{\mathbf{C}} e^{j \omega t} \cos k x\right]
\end{aligned}
$$

In Eq. (5.11), we have used the fact that $2 \cos x=\left(e^{j x}+e^{-j x}\right)$ and have absorbed $2 B$ into the new complex amplitude constant (phasor), $\widehat{\mathbf{C}}$, that will not be determined until the initial conditions have been specified.

As was done before for strings, setting $F_{x}(L, t)$ to zero quantizes the values of the wavenumber that satisfy both boundary conditions simultaneously. 


$$
F_{x}(L, t)=-E S_{x}\left(\frac{\partial \xi}{\partial x}\right)_{x=L}=E S_{x} k_{n} C_{n} \sin \left(k_{n} L\right) e^{j \omega_{n} t}=0
$$

This results in the same set of normal mode frequencies as the case for the fixed-fixed string in Eq. (3.20). These correspond to integer multiples of half-wavelengths between the ends of the bar.

$$
\begin{aligned}
\xi_{n}(x, t) & =\mathfrak{R} e\left[\widehat{\mathbf{C}}_{\mathbf{n}} \cos \left(k_{n} x\right) e^{j \omega_{n} t}\right] \\
\text { with } k_{n} & =\frac{n \pi}{L} \Rightarrow f_{n}=n \frac{c_{B}}{2 L} ; \quad n=1,2,3, \ldots
\end{aligned}
$$

Once again, there is a harmonic series of normal mode frequencies. Of course, in this case, there are displacement and velocity anti-nodes at both ends of the bar instead of velocity nodes at the ends, as was the case for the fixed-fixed string. For our standing wave solutions in Eq. (5.13), the displacement is proportional to $\cos \left(k_{n} x\right)$, but for the standing wave on a fixed-fixed string, $y_{n}(x) \propto \sin \left(k_{n} x\right)$, as shown in Eq. (3.21).

The fundamental free-free longitudinal mode of a very large aluminum bar, $3 \mathrm{~m}$ in length, with a mass of $2300 \mathrm{~kg}$, was used in an attempt to detect gravitational waves. ${ }^{1}$ [1] Using Young's modulus and the mass density of aluminum at room temperature, the speed of longitudinal waves $c_{L}=5510$ $\mathrm{m} / \mathrm{s}$, making $f_{1} \cong 860 \mathrm{~Hz}$. In operation, the bar was cooled below $4.2 \mathrm{~K}$ to permit the use of a superconducting quantum interferometer (SQUID) that measured the motion of a simple harmonic oscillator, tuned to $f_{1}$. The SQUID was attached to one end of the bar through a simple harmonic oscillator attached to one "free" end to amplify its motion by the $Q$ of the harmonic oscillator. That raised the resonance frequency to $904 \mathrm{~Hz}$, due to the increase in Young's modulus with decreasing temperature. The quality factor of the cryogenically cooled, freely suspended bar was enormous: $Q \cong 10^{9}[2]$.

Although harder to achieve in practice, an ideal fixed-fixed bar, with $\xi(0)=\xi(L)=0$, would also have integer multiple half-wavelengths between the ends of the bar, resulting in a harmonic series of normal mode frequencies. The displacement functions, $\xi_{n}(x, t)$, have the same functional form as the fixed-fixed string given in Eq. (3.12).

Like the solutions for the fixed-free string in Eqs. (3.23) and (3.24), a clamped-free bar that executes longitudinal vibrations will exhibit the same series of only odd-harmonic standing wave modes, corresponding to odd integer multiples of a quarter wavelengths between the fixed end at $x=0$ and the free end at $x=L$, where $(\partial \xi / \partial x)_{L}=0$.

$$
\begin{aligned}
\frac{\partial \xi(L, t)}{\partial x} & =k_{n} \widehat{\mathbf{C}}_{n} \cos \left(k_{n} L\right) e^{j \omega_{n} t}=0 \\
\cos k_{n} L & =0 \Rightarrow k_{n} L=\left(\frac{2 n-1}{2}\right) \pi \Rightarrow \omega_{n}=\left(\frac{2 n-1}{2}\right) \frac{\pi c_{B}}{L} \\
\Rightarrow \lambda_{n} & =\frac{4 L}{2 n-1} \quad \text { or } \quad L=(2 n-1) \frac{\lambda_{n}}{4} \quad \text { for } \quad n=1,2,3, \ldots
\end{aligned}
$$

\footnotetext{
${ }^{1}$ Such gravitational wave detectors are called "Weber bars" in honor of the first attempt to use a longitudinally resonant bar to detect gravitational waves that was made by J. Weber. Weber claimed to detect gravitational waves in an article entitled "Gravitational-Wave-Detector Events," Phys. Rev. Lett. 20, 1307-1308 (1968), although his claim could not be substantiated.
} 


\subsubsection{Longitudinal Waves in Bulk Solids}

As the ratio of the wavelength of the sound to the transverse dimensions of the bar becomes smaller, the compressive stresses associated with the longitudinal wave cannot be (partially) relieved by the bulge of the bar induced through Poisson's ratio. When the transverse dimensions of the sample substantially exceed the wavelength of the longitudinal wave, the modulus that provides the restoring force in Eq. (5.2) is no longer Young's modulus, $E$, but becomes the modulus of unilateral compression (also known as the dilatational modulus), $D>E$ (see Fig. 4.4). The propagation speed for a longitudinal wave in "bulk material," $c_{L}$, is higher than the bar speed.

$$
c_{L}=\frac{\omega}{k}=\sqrt{\frac{D}{\rho}}>c_{B}
$$

Expressing $D$ in terms of $E$ and $\nu$ (see Table 4.1) allows the ratio of the bulk longitudinal wave speed, $c_{L}$, to the thin bar speed, $c_{B}$, to be expressed in terms of Poisson's ratio.

$$
\frac{c_{L}}{c_{B}}=\frac{\sqrt{D / \rho}}{\sqrt{E / \rho}}=\sqrt{\frac{(1-\nu)}{(1+\nu)(1-2 \nu)}}
$$

Poisson's ratio for most common metals is about $\nu \cong 1 / 3$, so the sound speed ratio in metals is $c_{L} / c_{B} \cong$ $(3 / 2)^{1 / 2} \cong 1.2$, about a $20 \%$ higher speed for longitudinal waves in bulk materials, where transverse stresses cannot be relieved, in part, by "bulge."

\subsubsection{The Quartz Crystal Microbalance}

The quartz crystal microbalance (QCM) is used to "weigh" very thin films, such as those deposited on microelectronic circuits by vacuum evaporation of metals (frequently gold). This is accomplished by monitoring the change in the resonance frequency of the vibrating piezoelectric sample. As will be shown in this section, the deposition of thin films that have thicknesses that are on the order of a single atomic layer can be detected by monitoring the resonance frequency shift.

In exact analogy with the mass-loaded string (see Sect. 3.6), a bar or plate with a mass "attached" to the free face will require that the bar or plate provide the force necessary to accelerate the mass load adsorbed or plated onto the free face that is moving with longitudinal displacement, $\xi(L) e^{j \omega t}$.

$$
F_{x}(L, t)=M\left(\frac{\partial^{2} \xi}{\partial t^{2}}\right)_{x=L}=-D S_{x}\left(\frac{\partial \xi}{\partial x}\right)_{x=L}
$$

In Eq. (5.17), the modulus of unilateral compression, $D$, is used to relate stress and strain because we will assume that the quartz plate (usually a disk) has a diameter, $2 a$, that may be one hundred times the plate's thickness, $t=L$. Since the wavelength for the fundamental vibrational mode of a free-free plate is $\lambda=2 t$, and $t \ll a$, it is clear that the use of Young's modulus in this case would be incorrect. We will treat the "rear" of the disk as being suspended in a vacuum, hence free at $x=0$.

$$
\xi(x, t)=\mathfrak{R} e\left[\widehat{\mathbf{C}} \cos (k x) e^{j \omega t}\right]
$$

The face at $x=L$ will be mass-loaded by the deposition of a thin film with mass, $M_{\text {film }}$, so that Eq. (5.18) can be substituted into Eq. (5.17) to determine the quantization condition on $k_{n}$. 
Fig. 5.2 Graph of the transcendental Eq. (5.20) over a small region near the origin with $M_{\text {film }}$ /

$M_{\text {disk }}=0.10$ is shown as the dashed line. The tangent function, shown as the solid line, has a slope $=1.0$ near $\left(k_{n} L\right)=0$ or $\left(k_{n} L\right)=\pi$, so the first intersection of the dashed line and the tangent occurs at a value of $\left(k_{n} L\right)$ that is slightly less than $\pi$ by a small amount, $\Delta$

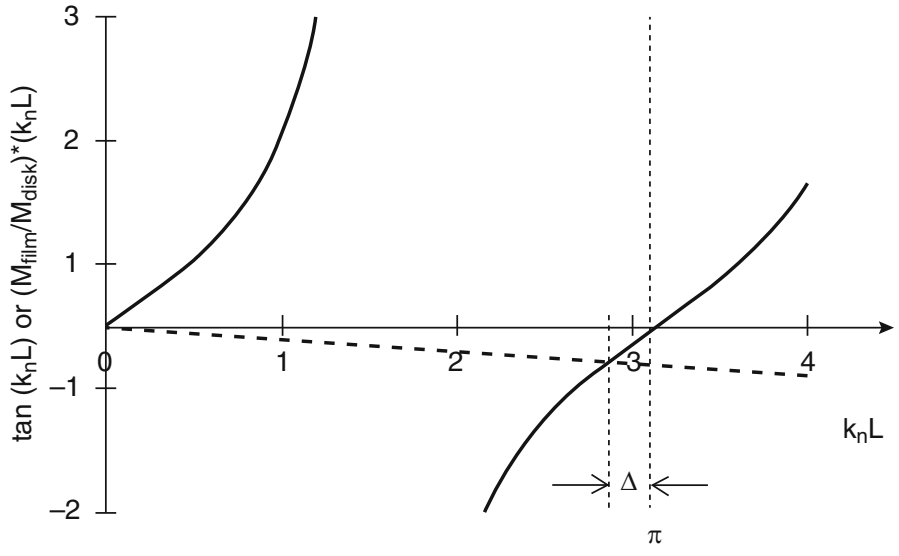

Using the fact that $c_{L}^{2}=\omega^{2} / k^{2}=D / \rho$, Eq. (5.19) can be written in a form similar to the quantization condition for the wavenumbers, $k_{n}$, of a fixed, mass-loaded string that was given in Eq. (3.68).

$$
\tan \left(k_{n} L\right)=\frac{-\omega^{2} M_{\text {film }}}{D S_{x} k_{n}}=-\frac{k_{n}^{2} M_{\text {film }} D}{k_{n} D S_{x} \rho} \frac{L}{L}=-\frac{M_{\text {film }}}{M_{\text {disk }}}\left(k_{n} L\right)
$$

This transcendental equation is plotted in Fig. 5.2 with $M_{\text {film }} / M_{\text {disk }} \ll 1$.

For a very small mass loading, $M_{\text {fim }} / M_{\text {disk }} \ll 1$, the first intersection of $\tan \left(k_{n} L\right)$ and the straight line, $\left(-M_{\text {film }} / M_{\text {disk }}\right)\left(k_{n} L\right)$, occurs at a value of $k_{n} L$ that is slightly less than $\pi$ by an amount $\Delta$, as shown in Fig. 5.2. We expect this decrease in the wavenumber (hence, resonant frequency), since the massloaded frequency should be lower than the normal mode frequency for its free-free value, $\left(k_{l} L\right)=\pi$. Since the slope of $\tan x$ at the zero-crossings is unity, $\Delta \cong \pi\left(M_{\text {film }} / M_{\text {disk }}\right) \Rightarrow \delta k_{1} \cong-(\pi / L)\left(M_{\text {film }} /\right.$ $M_{\text {disk }}$ ). Using this approximation (which, of course, becomes progressively better as the thickness of the film decreases), the relative shift in the wavenumber of the fundamental mode is $\delta k_{1} / k_{1}$, where $k_{l} \cong$ $(\pi / L)$. The relative frequency shift, $\delta f_{1} / f_{1}$, can be related to the film thickness, if the mass densities of the disk, $\rho_{\text {disk }}$, and the deposited film material, $\rho_{\text {film }}$, are known.

$$
\frac{\delta f_{1}}{f_{1}}=\frac{\delta k_{1}}{k_{1}}=-\frac{\pi}{L} \frac{M_{\text {film }}}{M_{\text {disk }}}\left(\frac{L}{\pi}\right)=-\left(\frac{\rho_{\text {film }}}{\rho_{\text {disk }}}\right) \frac{t_{\text {film }}}{L}
$$

This result is rather easy to interpret. This frequency shift is equivalent to the frequency change that would result if the disk was made longer by the thickness, $\delta L$, of the adsorbed film, if the adsorbed film had the same density as the density of the disk: $\delta L=\left(\rho_{\text {film }} / \rho_{\text {disk }}\right) t_{\text {film }}$, so $-\delta f_{l} / f_{1}=\delta L / L$.

A simple example will illustrate the sensitivity of this method. We start by calculating the thickness of a monolayer of gold atoms. The atomic weight of gold is $M_{A u}=197$ a.m.u. $=197 \mathrm{gm} / \mathrm{mole}$, and its mass density is $\rho_{A u}=19.3 \mathrm{gm} / \mathrm{cm}^{3}$. The cube root of the number of gold atoms $\left(N_{A u}\right)^{1 / 3}$ in a volume $V$ of a golden cube that is $1 \mathrm{~cm}$ along each edge will yield the spacing between layers of gold atoms, where Avogadro's number is $N_{A} \equiv 6.02214076 \times 10^{23}$ particles $/$ mole.

$$
\sqrt[3]{N_{A u}}=\left(\frac{\rho_{A u} V}{M_{A u}} N_{A}\right)^{1 / 3}=3.89 \times 10^{7} / \mathrm{cm}
$$


With $3.89 \times 10^{7}$ layers per centimeter, the average thickness of a single gold monolayer is $t_{A u}=2.57 \times 10^{-10} \mathrm{~m}=2.57 \AA^{2}$

Assume that a disk of piezoelectric quartz is used that is $L=t=0.50 \mathrm{~mm}$ thick and is vibrating in its fundamental free-free mode in a vacuum. The frequency of that mode is $f_{l}=c_{L} / 2 L$, where $c_{L}=5750 \mathrm{~m} / \mathrm{s}$ for quartz, so $f_{l}=5.75 \mathrm{MHz}$. Equation (5.21) can be used to calculate the decrease in the frequency of the fundamental mode, $-\delta f_{1}$, due to adsorption of a single monolayer of gold.

$$
\delta f_{1}=f_{1}\left(\frac{\rho_{\text {Au }}}{\rho_{\text {quartz }}}\right)\left(\frac{t_{A u}}{L_{\text {quartz }}}\right)=-5.75 \mathrm{MHz}\left(\frac{19.3}{2.65}\right)\left(\frac{2.57 \times 10^{-10}}{5 \times 10^{-4}}\right)=-21.5 \mathrm{~Hz}
$$

A frequency shift of $21.5 \mathrm{~Hz}$ is easy to detect. For this example, $-\delta f_{1} / f_{1}=3.74 \times 10^{-6}$, or 3.74 parts per million (ppm) for addition of a single atomic layer of gold.

One common example is the control of metal deposition on integrated circuit "chips." Gold is often "evaporated" in a vacuum system to deposit conduction paths between circuit elements and pin pads used to connect the integrated circuit to a printed circuit board. Monitoring the change in frequency of a quartz microbalance is an accurate way to determine when the desired thickness has been achieved.

\subsubsection{Bodine's "Sonic Hammer"}

At this point in the analysis, we would normally address the response of the driven system. Since the analogies between longitudinal vibrations in a thin bar and the transverse vibrations of a string are seen to be exact, we will focus on an interesting application instead. At the opposite extreme in size from the use of longitudinal resonance to weigh thin films with better than microgram precision, we will now focus on a method of driving pilings weighing several metric tons by exciting a standing wave resonance in "thin" bars. Shown below in Fig. 5.3 (left) is a construction site where a piling is to be driven into the ground by excitation of the longitudinal standing wave resonance of the pile.

An ordinary pile driver raises and releases a mass to drive (i.e., hammer) the pile into the ground, effectively launching a longitudinal pulse that travels down the pile until it reaches the end that is in contact with the earth and where the pulse reflection transmits a force that promotes penetration of the pile. Such "drop hammers" used for pilings of the size shown in Fig. 5.3 (right) will require masses in the range of several metric tons, typically $1500-4000 \mathrm{~kg}$, dropped from heights of 2 to $3 \mathrm{~m}$ above the piling. Each impact releases between $30 \mathrm{~kJ}$ and $120 \mathrm{~kJ}$ per blow. Assuming about one blow every $2 \mathrm{~s}$, using an average of $60 \mathrm{~kJ} / \mathrm{blow}$, deposits an average power of $30 \mathrm{~kW} \cong 40 \mathrm{hp}$ to the pile. ${ }^{3}$

A "sonic hammer," exciting the pile at its longitudinal resonance frequency, stores vibrational (or strain) energy in the pile and only has to replace the energy delivered to the earth to maintain steady-state operation at constant vibratory amplitude. The maximum stored potential energy $(P E)_{\max }$ can be calculated in the usual way for a bar of cross-sectional area, $S_{x}$; length, $L$; and volume, $V=S_{x} L$, using Eq. (1.22) or Eq. (2.14). The maximum vibrational strain amplitude is $\varepsilon_{x x}$ and $\xi=\varepsilon_{x x} d x$ is defined in Eq. (5.1).

\footnotetext{
${ }^{2}$ This is a slight underestimate because the crystalline structure of gold is face-center cubic. The accepted atomic diameter for gold is $2.88 \AA$.

${ }^{3}$ One horsepower [hp] is defined as $745.7 \mathrm{~W}$.
} 

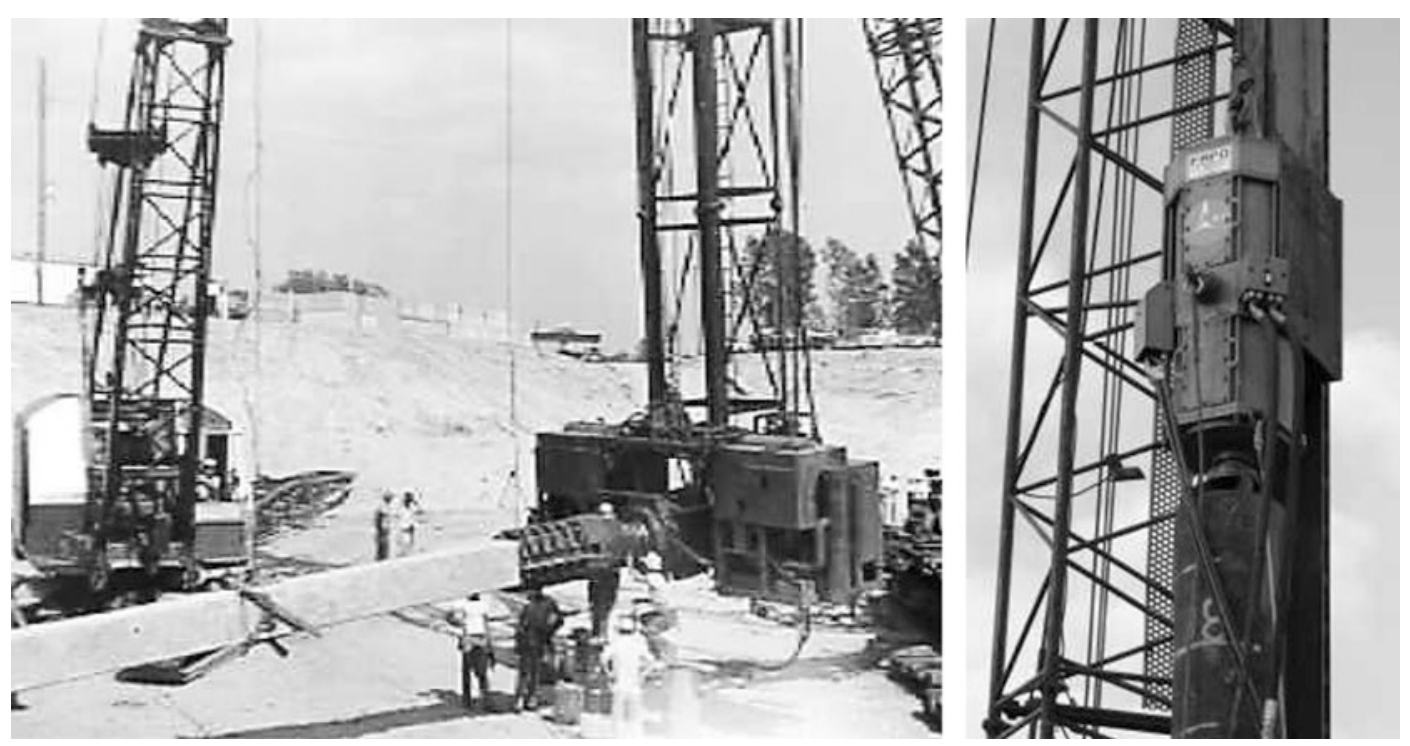

Fig. 5.3 (Left) Job site during preparation to drive a pile using resonant excitation provided by two engines from a Sherman tank. The engines provided counterrotation of eccentric masses (see Fig. 5.4) that drove the pile at its longitudinal standing wave resonance frequency. (Right) A $140 \mathrm{~kW}(200 \mathrm{HP})$ resonant driver, mounted on a $60 \mathrm{~cm}$ diameter steel pile, that uses a high-speed hydraulic valve to drive the pile at resonance. (Photographs courtesy of Matthew Janes)

$$
(P E)_{\max }=-\int_{0}^{L} F_{x} d \xi=S_{x} \int_{0}^{L} E \varepsilon_{x x}^{2} d x=S_{x} E \int_{0}^{L} \varepsilon_{x x}^{2} \cos ^{2}\left(\frac{n \pi x}{L}\right) d x=\frac{E \varepsilon_{x x}^{2}}{2} \quad V
$$

This analysis will be applied to a steel pipe with wall thickness of $22 \mathrm{~mm}$ and outside diameter of $30.0 \mathrm{~cm}$, that is, $17.8 \mathrm{~m}$ long, with a mass of $2668 \mathrm{~kg}$ [3]. With resonant excitation, the pipe penetrated over $9 \mathrm{~m}$ in $14 \mathrm{~min}$. A drop hammer could sink the first few meters of pipe with $60 \mathrm{blows} / \mathrm{m}$, but at greater depths, 150-200 blows/m were required. For $9 \mathrm{~m}$ penetration, over 1000 blows were required taking over $40 \mathrm{~min}$.

More importantly, for construction in dense urban locales, the ground vibrations due to the use of a drop hammer exceed the limit that can cause damage to existing structures nearby. In a similar experiment, ground vibrations from a drop hammer measured $10 \mathrm{~m}$ from the pile showed ground accelerations of $1.75 \mathrm{~m} / \mathrm{s}^{2}$, while the resonant drive produced accelerations of $0.25-0.30 \mathrm{~m} / \mathrm{s}^{2}$ at the same measurement distance [3].

At the job site, construction workers can measure the amplitude of vibration near a velocity antinode by taking a chalk stick and drawing a line along the pile's circumference. Since the surface of the pile is vibrating up and down, this draws a sinusoid and provides a visual representation of the peak-topeak vibrational displacement, $2 \xi_{o}$. For this type of installation, $2 \xi_{o} \cong 6 \mathrm{~cm}$, and the resonance frequency is roughly $100 \mathrm{~Hz}$. The maximum fully reversing strain, $\varepsilon_{x x} \cong 0.03 / 17.8=1.7 \times 10^{-3}$, is well below the elastic limit of steel. The total volume of steel in the pipe is $V=0.37 \mathrm{~m}^{3}$. Using $E_{\text {steel }}=195 \mathrm{GPa}$, the total stored potential energy given by Eq. $(5.24)$ is $(P E)_{\max } \cong 100 \mathrm{~kJ}$, fairly close to the energy in a single hammer drop, except this energy is available continuously.

The rate at which this energy is delivered to the ground depends upon the quality factor of the resonance, usually dominated by the losses at the entry cavity. In this demonstration, the average 
power delivered to the pipe $<\Pi>_{t} \cong 50 \mathrm{~kW} .{ }^{4}$ Using Eq. (2.70), the average quality factor for the pipe's longitudinal vibration can be calculated.

$$
Q=\frac{2 \pi f E_{\text {stored }}}{\langle\Pi\rangle_{t}} \cong 1300
$$

In addition to the reduction in surrounding vibrations for the pile driving application, this resonant vibrational strategy has other applications. In particular, since the resonant method does far less damage to the surrounding material, it is a good way to take geological core samples. It is particularly well-suited to ice core sampling, since very little damage is done to the ice and it takes less time to cut the core (which is important in Arctic weather conditions). This technique has also been used for tunneling in the construction of the Bay Area Rapid Transit (BART) subway system around San Francisco, Oakland, and Berkeley in California. A similar approach has also been developed for rock crushing [5], although that device used resonant excitation of the chute to shake the crushing balls against the material, not longitudinal waves in bars.

There have been problems with these longitudinally resonant pile drivers. The early drivers were prone to failure due to the enormous cyclic loading of the bearings and gears shown in Fig. 5.4. In some cases, crane operators were responsible for the failure because they could not believe that the piles would sink so quickly and did not lower the drive mechanism quickly enough for the ground to absorb the vibrational energy. The reduced cutting load caused the $Q$ of the pile to become so large that the increased vibrational amplitude damaged the drivers. Hydraulic actuators have largely removed that difficulty. Since the resonance frequency changes continuously as the pile or pipe enters the ground, a control system can be employed to keep the system "tuned" to the longitudinal bar resonance as its length changes and as it encounters differing soils.
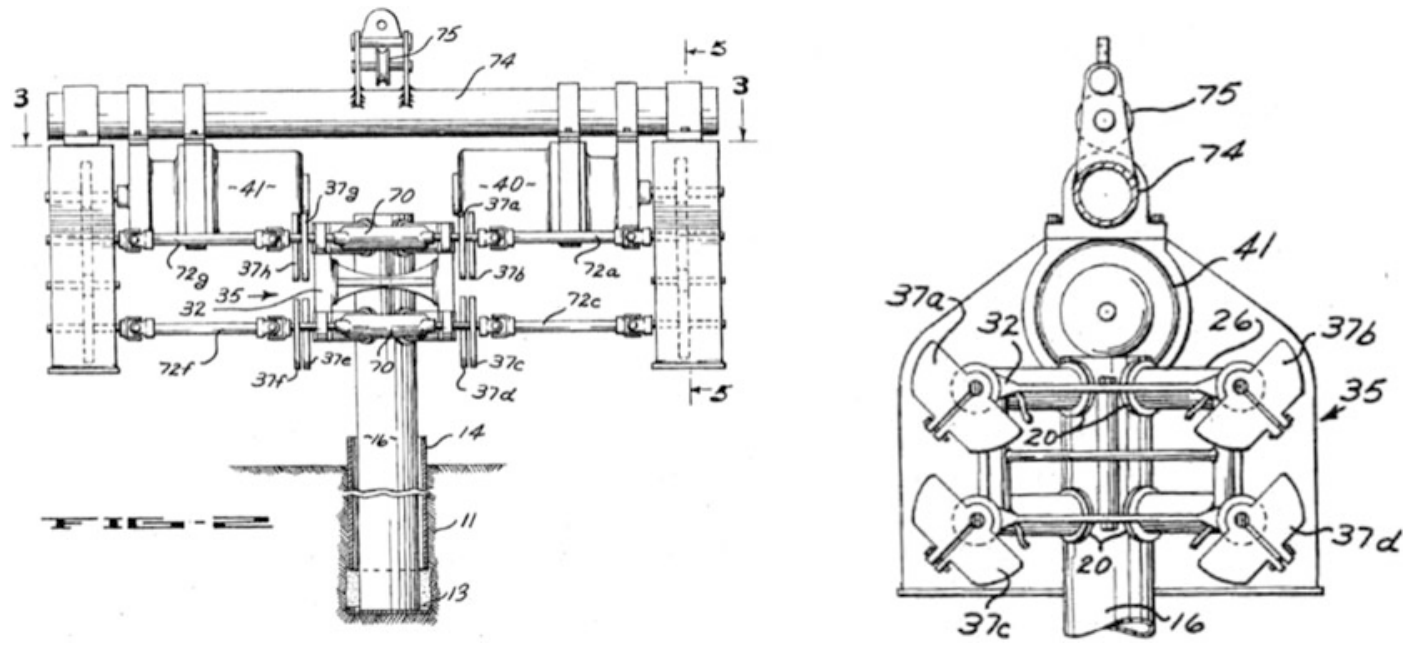

Fig. 5.4 (Left) Mechanical driving system attached to the top of the piling for resonant excitation of longitudinal standing waves in a construction. Motors (40 and 41) drive shafts (72), through gearboxes that are connected to the eccentrically mounted masses (37) to provide the longitudinal excitation to the top of the piling (16). (Right) View showing the rotating eccentric masses (37) [4]

\footnotetext{
${ }^{4}$ Using hydraulic fluid at $17 \mathrm{MPa}$, with an average flow rate of $U=4.5 \mathrm{l} / \mathrm{s}=4.5 \times 10^{-3} \mathrm{~m}^{3} / \mathrm{s}$, the hydraulic power $<\Pi\rangle_{t}=(\Delta p) U \cong 76 \mathrm{~kW}$.
} 


\subsection{Torsional Waves in Thin Bars}

The same procedure that has been used for the longitudinal waves can be applied used to calculate the speed of torsional wave propagation in thin bars or tubes of circular cross-section and to calculate the speed of shear waves in bulk solids. Since we have already calculated the torque required to twist a circular rod or tube of length, $L$, by an angle, $\phi$, in Eq. (4.50), that result can be applied to a differential length, $d x$, of a solid rod of radius, $a$. The difference in the twist at $x$ and at $x+d x$ is $d \phi$, so $\phi / L$ becomes $\partial \phi / \partial x$.

$$
N(x)=G \frac{\pi a^{4}}{2} \frac{\partial \phi}{\partial x}
$$

The difference in the torque between the ends of the differential element is then approximated by a Taylor series.

$$
d N(x)=N(x+d x)-N(x)=\left(\frac{\partial N}{\partial x}\right) d x=G \frac{\pi a^{4}}{2}\left(\frac{\partial^{2} \phi}{\partial x^{2}}\right) d x
$$

For a circular rod with constant mass density, $\rho$, the mass of the differential element is just $d m=$ $\pi \rho a^{2} \mathrm{~d} x$. The polar moment of inertia, $I_{\text {disk }}$, of a circular disk is $m a^{2} / 2$, so for the differential element, $I_{\text {disk }}=(\pi / 2) \rho a^{4} d x$.

Newton's Second Law guarantees that the net torque is equal to the moment of inertia times the angular acceleration.

$$
d N(x)=G \frac{\pi a^{4}}{2}\left(\frac{\partial^{2} \phi}{\partial x^{2}}\right) d x=d I_{d i s k}\left(\frac{\partial^{2} \phi}{\partial t^{2}}\right) d x=\frac{\pi}{2} \rho a^{4}\left(\frac{\partial^{2} \phi}{\partial t^{2}}\right) d x
$$

This result can be rearranged to yield a torsional wave equation for a rod of circular cross-section that introduces the shear wave speed, $c_{S}$.

$$
\frac{\partial^{2} \phi}{\partial x^{2}}-\frac{\rho}{G} \frac{\partial^{2} \phi}{\partial t^{2}}=0 \Rightarrow c_{S}=\sqrt{\frac{G}{\rho}}
$$

For a circular bar of radius, $a$, the propagation speed, $c_{S}$, for torsional waves does not depend upon the radius of the bar. In fact, since the volume is unchanged by shear deformation, it does not even require that the radius, $a$, be much less than $\lambda$, as was the case for longitudinal waves. Since there is no volume change, there is no "bulge" regardless of the ratio of the sample size to the wavelength. Bulk shear waves also propagate at $c_{S}$.

The torsional wave speed in a thin bar does depend on the shape of the bar's cross-section. We can generalize the torque equation that was written in Eq. (5.26) to shapes that are not circularly symmetric by introducing a torsional rigidity, $J$, which will depend upon the bar's cross-sectional shape [6].

$$
N(x)=J \frac{\partial \phi}{\partial x}
$$

Values for $J / G$ are provided in the second column of Table 5.1, so for our circular bar example, $J_{\text {disk }}=$ $\pi G a^{4} / 2$, as expressed in Eq. (5.26). Similarly, the polar moment of inertia, $I$, can be evaluated for the corresponding cross-sectional shape as provided in the third column of Table 5.1. Both $J$ and $I$ can be substituted into the wave equation to produce a torsional wave speed, $c_{S S}$, that is dependent upon the cross-sectional shape of a thin bar with transverse dimensions that are much less than the length [7]. 
Table 5.1 Both the torsional rigidity, $J$, and the moment of inertia, $I$, of a rod depend upon the shape of its cross-section

\begin{tabular}{l|l|l|l}
\hline Cross-section & $N L / G \equiv J / G$ & $I$ & Torsional speed \\
\hline $\begin{array}{l}\text { Circular } \\
\text { Radius }=a\end{array}$ & $\frac{\pi a^{4}}{2}$ & $\frac{\pi a^{4}}{2}$ & $c_{S}$ \\
\hline $\begin{array}{l}\text { Ring } \\
\text { Outer }=a_{\text {out }} \\
\text { Inner }=a_{\text {in }}\end{array}$ & $\frac{\pi\left(a_{\text {out }}^{4}-a_{\text {in }}^{4}\right)}{2}$ & $\frac{\pi\left(a_{\text {out }}^{4}-a_{\text {in }}^{4}\right)}{2}$ & $c_{S}$ \\
\hline $\begin{array}{l}\text { Elliptical } \\
\text { Semi-major }=a\end{array}$ & $\frac{\pi a^{3} b^{3}}{a^{2}+b^{2}}$ & $\frac{\pi}{4} a b\left(a^{2}+b^{2}\right)$ & $\frac{2 a b}{a^{2}+b^{2}} c_{S}$ \\
\hline $\begin{array}{l}\text { Semi-minor }=b \\
\text { Edge }=w\end{array}$ & $9 w^{4} / 64$ & & \\
\hline $\begin{array}{l}\text { Rectangular } \\
\text { Thickness }=t \\
\text { Width }=w\end{array}$ & $\frac{w t^{3}}{16}\left[\frac{16}{3}-3.36 \frac{t}{w}\left(1-\frac{t^{4}}{12 w^{4}}\right)\right]$ & $\frac{w^{4} / 6}{2 w\left(t^{2}+w^{2}\right)}$ & $\sqrt{\frac{27}{32}} c_{S} \cong 0.92 c_{S}$ \\
\hline $\begin{array}{l}\text { Equilateral triangle } \\
\text { Edge }=a\end{array}$ & $\frac{a^{4} \sqrt{3}}{80}$ & $\frac{2 t}{w} c_{S}$ if $t<<w$ \\
\hline
\end{tabular}

$$
\frac{\partial^{2} \phi}{\partial x^{2}}-\frac{J}{\rho I} \frac{\partial^{2} \phi}{\partial t^{2}}=0 \quad \Rightarrow \quad c_{S S}=\sqrt{\frac{J}{\rho I}}
$$

Table 5.1 summarizes the speed of torsional waves on bars of various cross-sectional shapes by taking the ratio of the torsional stiffness-length product that relates static torque, $N$, to the shear modulus, $G$, and dividing by the polar moment of inertia, $I$. These factors for a rod and a hollow tube were calculated in Sect. 4.3.5 and given in Eq. (4.45). The other stiffness-length products were taken from Table 20 in Roark [8].

The torsional stiffness-length product (or ratio of torsional rigidity to shear modulus) is provided by the ratio of the torque, $N$, divided by the shear modulus, $G$. The ratio of torsional stiffness-length product to the moment of inertia, $I$, provides the constant multiplying the shear wave speed, $c_{S}$, for each bar cross-sectional shape.

\subsection{Flexural Waves in Thin Bars}

The last of the three independent vibrational modes of thin bars mentioned in the introduction is the flexural or bending modes. We will again follow the same procedure to determine their behavior, but that path will take us to a description that is fundamentally different from the results we have derived for transverse waves on strings or the longitudinal and torsional waves in thin bars. As was done for the case of transverse waves, we seek an expression for the vertical force, $F_{v}(x)$, on a differential element that can be related to its inertia via Newton's Second Law. We start from the expression for the bending moment, $\mathfrak{M}$, in the limit of small displacements, which is related to the curvature in Eq. (4.29).

$$
\mathfrak{M}=-E S \kappa^{2} \frac{\partial^{2} y}{\partial x^{2}}
$$

The square of the radius of gyration, $\kappa^{2}$, is defined in Eq. (4.29).

Using the fact that the moment is the torque produced by an applied force times its perpendicular distance from the reference point, we can calculate the vertical force that creates the moment in Eq. (5.32). It can be calculated by placing the reference location at $x=0$. In that way, the vertical force applied at $x=0$ has no moment. The torque due to the shear force at $x+d x$ will be the product of $F_{y}(x+d x)$ and its "lever arm," $d x$. 


$$
F_{v}(x+d x) d x=\mathfrak{M}(x+d x)-\mathfrak{M}(x)
$$

By our choice of $x=0$ as the reference location, $\mathfrak{M}(x)=0$. A Taylor series can then be exploited to express the moment of the vertical force, $F_{y}(x+d x) d x$, that will counteract the moment.

$$
F_{v}(x+d x) d x=\frac{\partial \mathfrak{M}}{\partial x} d x=-E S_{x} \kappa^{2} \frac{\partial^{3} y}{\partial x^{3}} d x
$$

This expression for the vertical component of the force plays the same role as Eq. (3.2) that relates the slope of the string to the vertical force on a string element, or Eq. (5.2) that relates the stress in the bar to the longitudinal force on the bar element. Of course, Eqs. (3.2) and (5.2) related these forces to a first derivative with respect to position of the displacement from equilibrium, while Eq. (5.34) relates the vertical force to the third derivative of the displacement.

We determine the net vertical force acting on our differential element in the usual way.

$$
d F_{v}=F_{v}(x+d x)-F_{v}(x)=\frac{\partial F_{v}}{\partial x} d x=-E S_{x} \kappa^{2} \frac{\partial^{4} y}{\partial x^{4}} d x
$$

This result is also known as the Euler-Bernoulli beam equation after Leonhard Euler and Daniel Bernoulli who first derived this result in the 1750s [9]. The combination of Young's modulus for the beam multiplied by the area and the radius of gyration is known as the flexural rigidity, $E S_{x} \kappa^{2}$, since it is a measure of the moment of the force necessary to deform the bar.

The mass of the differential element is $\rho S_{x} d x$, so Newton's Second Law produces the differential equation describing the bar's dynamic response to flexure.

$$
\rho S d x \frac{\partial^{2} y}{\partial t^{2}}=-E S_{x} \kappa^{2} \frac{\partial^{4} y}{\partial x^{4}} d x \Rightarrow \frac{\partial^{2} y}{\partial t^{2}}+\kappa^{2} c_{B}^{2} \frac{\partial^{4} y}{\partial x^{4}}=0
$$

The right-hand expression makes use of the propagation speed of longitudinal waves in thin bars, $c_{B}^{2}=E / \rho$, after cancellation of the common factors $\mathrm{d} x$ and $S_{x}{ }^{5}$

\subsubsection{Dispersion}

Equation (5.36) is a linear, fourth-order, homogeneous, partial differential equation that is clearly not the wave equation. Its solutions will not be just any functions of the argument $x \pm c t$, where $c$ is a constant (independent of frequency or wavelength) representing the propagation speed for all waves. Fortunately, Eq. (5.36) contains only even-order partial derivatives, so substitution of a complex exponential will provide a relation between frequency and wavenumber that is a real number. ${ }^{6}$ This relationship can be demonstrated by substitution of our usual rightward-going traveling wave solution, $y(x, t)=\mathfrak{R} e\left[\widehat{\mathbf{C}} e^{j(\omega t-k x)}\right]$, into Eq. (5.36) to solve the time-independent Helmholtz equation.

\footnotetext{
${ }^{5}$ This equation is only approximately correct since the moment is also opposed by the rotary inertia of the bar. The approximation is very good when $\lambda \gg a$, which is our current focus. The complete equation for the dynamics, which includes rotary inertia and shear, is derived in several references, for example, D. Ross, The Mechanics of Underwater Noise (Pergamon, 1976); ISBN 0-08-021182-8.

${ }^{6}$ As will be shown in Chap. 9 on dissipative hydrodynamics, this is not the case for equations that contain both odd- and even-order derivatives. The Navier-Stokes equation, describing viscous dissipation; the Fourier heat diffusion equation, describing thermal conduction losses; and the Schrodinger equation of quantum mechanics all contain both odd- and even-order derivatives that require complex numbers to relate frequency and wavenumber.
} 


$$
-\omega^{2} y+\kappa^{2} c_{B}^{2} k^{4} y=0 \Rightarrow k= \pm \sqrt{\frac{\omega}{\kappa c_{B}}}
$$

The most useful equation in acoustics is the relationship between frequency and wavelength: $f \lambda=\omega / k=c$. Up to this point, $c$ has been treated as a constant in all systems, irrespective of frequency or wavelength. This is not the case for solutions to Eq. (5.36). We can solve Eq. (5.37) for the ratio of frequency to wavenumber.

$$
\frac{\omega^{2}}{k^{2}}=\kappa^{2} c_{B}^{2} k^{2} \Rightarrow \frac{\omega}{k}= \pm\left(\kappa c_{B}\right) k= \pm 2 \pi \frac{\kappa c_{B}}{\lambda}
$$

The propagation speed for harmonic flexural waves of frequency, $\omega$, is inversely proportional to the wavelength of the disturbance.

In all our previous solutions to the dynamical response for systems described by the wave equation, $\omega / k$ is equal to a constant, $c$, that is identified as the speed of propagation for all wavelike disturbances, whether they are pulses or harmonic waves, of either the traveling or standing variety, independent of their frequency or wavelength. Equation (5.38) demonstrates that the ratio of frequency to wavenumber is no longer a constant that is independent of frequency for flexural waves on bars.

$$
c_{p h} \equiv \frac{\omega}{k}= \pm \sqrt{\kappa \omega c_{B}}
$$

Because there is no longer a single speed of propagation for all disturbances, we have defined the phase speed, $c_{p h} \equiv \omega / k$. This is the speed of propagation of wave fronts of any disturbance that is a pure tone, having a single frequency, $\omega$, and a single wavelength, $\lambda$, thus containing only one Fourier component. A wavelike disturbance that contains more than one Fourier component will not retain its shape as it propagates under the influence of Eq. (5.36), which is still a linear equation. Since the different frequency components of such a disturbance will travel with different speeds, Eq. (5.39) implies that the higher-frequency components will get ahead of the lower-frequency components, so the waveform produced by their superposition will change over time and distance.

The dependence of propagation speed on frequency or wavelength is known as dispersion. Dispersion of light waves was demonstrated experimentally by Newton [10] who used a glass prism to separate white light into its different constituent wavelengths. The angle of refraction of light passing through a transparent medium depends upon the ratio of the propagation speeds, in Newton's case, between air and glass. For glass, that propagation speed depends upon the wavelength of the light. In effect, the prism provided a Fourier analysis (64 years before the birth of Fourier) of the different frequency components (different colors) that superimpose to create white light. ${ }^{7}$ The genius of Isaac Newton was displayed by his use of a second prism to recombine the different colors to produce white light, thus demonstrating that the colors were not an experimental artifact.

Capillary waves on the surface of water have the same dispersion relation as flexural waves on thin bars [11], $c_{p h}=(2 \pi \sigma / \rho \lambda)^{1 / 2}$, where $\sigma$ is the surface tension of water. ${ }^{8}$ Like flexural waves on thin bars,

\footnotetext{
${ }^{7}$ The laws of electromagnetism, known as Maxwell's equations, which govern the propagation of light, are also linear differential equations.

${ }^{8}$ The full dispersion relation for surface waves on fluids produces the dispersion relation (below) that also includes the force of gravity $g$, which dominates the restoring force at long wavelengths, as well as surface tension (capillarity). When the wavelengths are long compared to the depth, $h$, so $k h \ll 1, \tanh (k h)$ is proportional to $k h$ and the speed is again dispersionless. On water, capillarity (surface tension, $\sigma$ ) dominates gravity for wavelengths less than about one-half centimeter.
}

$$
c_{p h}^{2}=\frac{\omega^{2}}{k^{2}}=\left(\frac{g}{k}+\frac{\sigma}{\rho} k\right) \tanh (k h)
$$




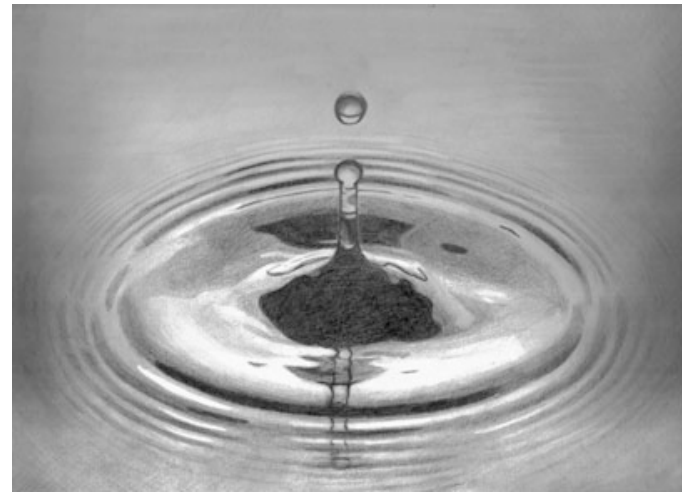

Fig. 5.5 This photograph of a water drop's splash clearly illustrates the dispersion of waves that have a phase velocity, $c_{p h}$, that is inversely related to the wavelength. The components of the disturbance with the shortest wavelengths have moved ahead of the longer-wavelength components. The dispersion for surface waves on water with wavelengths less than about $5 \mathrm{~mm}$ is dominated by surface tension. ${ }^{9}$

shorter wavelengths (i.e., higher frequencies) propagate faster than longer wavelengths. This is visible in Fig. 5.5, where a drop of water launches cylindrically diverging surface waves with the short wavelengths moving away from the source faster than the longer-wavelength components produced by the splash. ${ }^{9}$

\subsubsection{Flexural Wave Functions}

Capitalizing on our success with the substitution of a complex traveling waveform, $y(x, t)=$ $\mathfrak{R} e\left[\widehat{\mathbf{C}} e^{j(\omega t-k x)}\right]$, into Eq. (5.36) to produce the dispersion relations of Eqs. (5.37), (5.38), and (5.39), we will now seek a complete solution to Eq. (5.36) by the method known as separation of variables. By setting $y(x, t)=Y(x) T(t)$, the spatial dependence of the solution depends only upon $Y(x)$, and the temporal behavior depends only upon $T(t)$. Since Eq. (5.36) is a linear equation, we can still construct more complicated waveforms by the superposition of harmonic waves, so we will let $T$ $(t)=e^{j \omega t}$. Substitution of $T(t)$ into Eq. (5.36) converts it from a partial differential equation to an ordinary (forth-order) differential equation for $Y(x)$.

$$
\frac{d^{4} Y}{d x^{4}}=\frac{\omega^{2}}{\kappa^{2} c_{B}^{2}} Y=\frac{\omega^{4}}{c_{p h}^{4}} Y
$$

Letting $Y(x)=Y \mathrm{e}^{k x}$, a simple quartic expression is generated for the wavenumber $k: k^{4}=\left(\omega / c_{p h}\right)^{4}$. By taking the fourth root (see Sect. 1.5.2), we generate four solutions for $k= \pm \omega / c_{p h}$ and $\pm \mathrm{j} \omega / c_{p h}$. Those four solutions are superimposed in Eq. (5.41).

$$
\mathbf{y}(x, t)=\left[C_{1} e^{\left(\omega x / c_{p h}\right)}+C_{2} e^{-\left(\omega x / c_{p h}\right)}+C_{3} e^{j\left(\omega x / c_{p h}\right)}+C_{4} e^{-j\left(\omega x / c_{p h}\right)}\right] e^{j \omega t}
$$

\footnotetext{
${ }^{9}$ For ripple-tank demonstrations, the depth of the water is about $5 \mathrm{~mm}$. At that depth, the restoring forces of gravity and surface tension balance to produce a frequency-independent surface wave velocity, $c \cong 23 \mathrm{~cm} / \mathrm{s}$. [See M. J. Lighthill, Waves in Fluids (Cambridge, 1978); ISBN 052121689 3. Sec. 1.8 (Ripple-tank simulations)]
} 
Fig. 5.6 Schematic representation of four possible boundary conditions for flexure of a bar: (a) hinged, (b), clamped, (c) free, and (d) guided or sliding. From Han et al. [12]

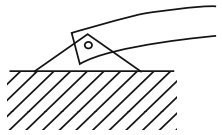

(a)

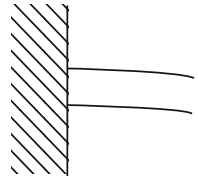

(b)

$$
y=0, \frac{\partial y}{\partial x}=0
$$

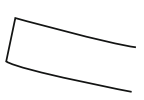

(c)

(d)

$$
y=0, \frac{\partial^{2} y}{\partial x^{2}}=0
$$

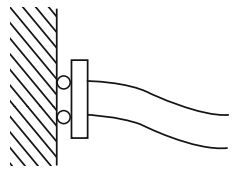

$$
\frac{\partial y}{\partial x}=0, \frac{\partial^{3} y}{\partial x^{3}}=0
$$

This result confirms our first solution, a right-going traveling wave, that was used to produce Eq. (5.37), corresponding to $C_{1}=C_{2}=C_{3}=0$ and $C_{4} \neq 0$. Linearity allows us to combine these exponential solutions to produce a solution that can be expressed as four independent trigonometric functions with real constants.

$$
\begin{aligned}
A & =\left(C_{1}+C_{2}\right) / 2, \quad B=\left(C_{1}-C_{2}\right) / 2, \quad C=\left(C_{3}+C_{4}\right) / 2, \text { and } D=\left(C_{3}-C_{4}\right) / 2 j . \\
y(x, t) & =\left[A \cosh \left(\frac{\omega x}{c_{p h}}\right)+B \sinh \left(\frac{\omega x}{c_{p h}}\right)+C \cos \left(\frac{\omega x}{c_{p h}}\right)+D \sin \left(\frac{\omega x}{c_{p h}}\right)\right] \cos (\omega t+\phi)
\end{aligned}
$$

Since these are solutions to a fourth-order differential equation, four boundary conditions must be specified to determine the four amplitude coefficients. The four most common "perfect" boundary conditions are clamped, free, hinged, and guided. They are summarized in Fig. 5.6.

If the bar is clamped, then the end cannot move in the vertical direction, so $y=0$. The clamped end of the bar must also approach that boundary with zero slope, so $(\partial y / \partial x)=0$. If the bar is free, then the boundary can exert no moments (torques), so from Eq. (5.32), $\left(\partial^{2} y / \partial x^{2}\right)=0$. The free boundary cannot provide any vertical forces, so from Eq. (5.34), $\left(\partial^{3} y / \partial x^{3}\right)=0$.

A hinged boundary (or knife-edged clamp) will also make $y=0$. Due to the hinge, the boundary can exert no torques, so $\left(\partial^{2} y / \partial x^{2}\right)=0$. Of course, the hinge applies vertical forces to keep $y=0$, so $\left(\partial^{3} y /\right.$ $\left.\partial x^{3}\right) \neq 0$. A guided or sliding boundary allows the beam to move in the vertical direction (i.e., $y \neq 0$ ) but constrains both the slope and the vertical force to be zero.

\subsubsection{Flexural Standing Wave Frequencies}

In Sect. 4.3.2, we calculated the deflection curve for a horizontal cantilevered beam that was clamped at $x=0(y=0$ and $\partial y / \partial x=0)$ and was loaded by a vertical force (e.g., a weight) at $x=L$ (see Galileo's illustration in Fig. 2.27). We will start this section by calculating the normal modes of oscillation for a cantilevered beam that is clamped at $x=0$ and is free at $x=L$. Using the trigonometric form of the general solution in Eq. (5.42) and applying the boundary condition at $y(0)=0, \sinh (x)$ and $\sin (x)$ are automatically zero, but $\cos (x)$ and $\cosh (x)$ are both equal to 1 , so $A=-C$. For the slope to vanish at $x=0, B=-D$, since $d(\sinh (x)) / d x=\cosh (x)$ and $d(\sin (x)) / d x=\cos (x)$. Suppressing the time dependence temporarily (no pun intended), the wave function for the cantilever beam can be written with only two arbitrary constants when it is clamped at $x=0$.

$$
y(x)=A\left[\cosh \left(\frac{\omega x}{c_{p h}}\right)-\cos \left(\frac{\omega x}{c_{p h}}\right)\right]+B\left[\sinh \left(\frac{\omega x}{c_{p h}}\right)-\sin \left(\frac{\omega x}{c_{p h}}\right)\right]
$$


Evaluation of the second and third derivatives of Eq. (5.43) at $x=L$ will provide the quantization of the allowable modal (normal mode) frequencies, $\omega_{n}$, and wavenumbers, $k_{n}$.

$$
\begin{aligned}
& A\left[\cosh \left(\frac{\omega_{n} L}{c_{p h}}\right)+\cos \left(\frac{\omega_{n} L}{c_{p h}}\right)\right]+B\left[\sinh \left(\frac{\omega_{n} L}{c_{p h}}\right)+\sin \left(\frac{\omega_{n} L}{c_{p h}}\right)\right]=0 \\
& A\left[\sinh \left(\frac{\omega_{n} L}{c_{p h}}\right)-\sin \left(\frac{\omega_{n} L}{c_{p h}}\right)\right]+B\left[\cosh \left(\frac{\omega_{n} L}{c_{p h}}\right)+\cos \left(\frac{\omega_{n} L}{c_{p h}}\right)\right]=0
\end{aligned}
$$

Both of the equations in Eq. (5.44) can be used to solve for $B$ in terms of $A$.

$$
B=A \frac{\sin \left(\frac{\omega_{n} L}{c_{p h}}\right)-\sinh \left(\frac{\omega_{n} L}{c_{p h}}\right)}{\cos \left(\frac{\omega_{n} L}{c_{p h}}\right)+\cosh \left(\frac{\omega_{n} L}{c_{p h}}\right)}=-A \frac{\cos \left(\frac{\omega_{n} L}{c_{p h}}\right)+\cosh \left(\frac{\omega_{n} L}{c_{p h}}\right)}{\sin \left(\frac{\omega_{n} L}{c_{p h}}\right)+\sinh \left(\frac{\omega_{n} L}{c_{p h}}\right)}
$$

After cancellation of the common factor of $A$, cross-multiplication of the left and right fractions in Eq. (5.45) produces a single equation that quantizes the normal mode frequencies, $\omega_{n}$.

$$
\sinh ^{2}\left(\frac{\omega_{n} L}{c_{p h}}\right)-\sin ^{2}\left(\frac{\omega_{n} L}{c_{p h}}\right)=\left[\cosh \left(\frac{\omega_{n} L}{c_{p h}}\right)+\cos \left(\frac{\omega_{n} L}{c_{p h}}\right)\right]^{2}
$$

Application of the trigonometric identities $\cos ^{2}(\theta)+\sin ^{2}(\theta)=1$ and $\cosh ^{2}(\theta)-\sinh ^{2}(\theta)=1$ produces a workable transcendental equation that can be solved for the normal mode frequencies.

$$
\cosh \left(\frac{\omega_{n} L}{c_{p h}}\right) \cos \left(\frac{\omega_{n} L}{c_{p h}}\right)=-1
$$

The solution of Eq. (5.47) is inconvenient because the hyperbolic cosine will diverge exponentially. The use of the following half-angle formulas can afford further simplification.

$$
\tan ^{2}\left(\frac{\theta}{2}\right)=\frac{1-\cos \theta}{1+\cos \theta} \text { and } \tanh ^{2}\left(\frac{\theta}{2}\right)=\frac{\cosh \theta-1}{\cosh \theta+1}
$$

Since the limits of the hyperbolic tangent for large arguments are \pm 1 , the following forms are both easier to graph and avoid the exponential divergence of the hyperbolic cosine.

$$
\operatorname{coth}^{2}\left(\frac{\omega_{n} L}{2 c_{p h}}\right)=\tan ^{2}\left(\frac{\omega_{n} L}{2 c_{p h}}\right) \quad \text { or } \quad \cot \left(\frac{\omega_{n} L}{2 c_{p h}}\right)= \pm \tanh \left(\frac{\omega_{n} L}{2 c_{p h}}\right)
$$

Figure 5.7 is a plot of the two functions on the right-hand side of Eq. (5.49) vs. their argument $x=\omega L / 2 c_{p h}$. For arguments of $x>4$, the value of $\pm \tanh (x) \cong \pm 1 . \operatorname{Cot}^{-1}(x)= \pm 1$ for $x=(2 n-1)(\pi /$ $4)$, so for $n \geq 3, \omega L / 2 c_{p h}=(2 n-1)(\pi / 4)$. An equation solver can be used to calculate the lowest two solutions to Eq. (5.49) that are $x_{1} \cong 1.1937(\pi / 4)$ and $x_{2} \cong 2.9884(\pi / 4)$.

Combining the solutions to Eq. (5.49) with the square of the phase speed in Eq. (5.39), the flexural normal mode frequencies, $f_{n}$, of the clamped-free bar can be written as a function of the geometry of the $\operatorname{bar}(L$ and $\kappa)$, Young's modulus, and the mass density of the beam's material, $c_{B}=(E / \rho)^{1 / 2}$.

$$
f_{n}=\frac{\omega_{n}}{2 \pi}=\frac{\pi}{8} \frac{c_{B} \kappa}{L^{2}}\left(1.1937^{2}, 2.9884^{2}, 5.0005^{2}, 7^{2}, 9^{2}, \ldots\right)
$$

It is worthwhile to point out the significant differences in this expression from those for the normal mode frequencies of the vibrating string and other systems that obey wave equations of the form of 
Fig. 5.7 The two terms on the right-hand side of Eq. (5.49) are plotted as a function of $x=\omega L / 2 c_{p h}$. The hyperbolic tangents are shown as solid lines and the dash-dot line is the cotangent. Their intersections define the normal mode frequencies, $\omega_{n}$, for a clamped-free (cantilevered) beam

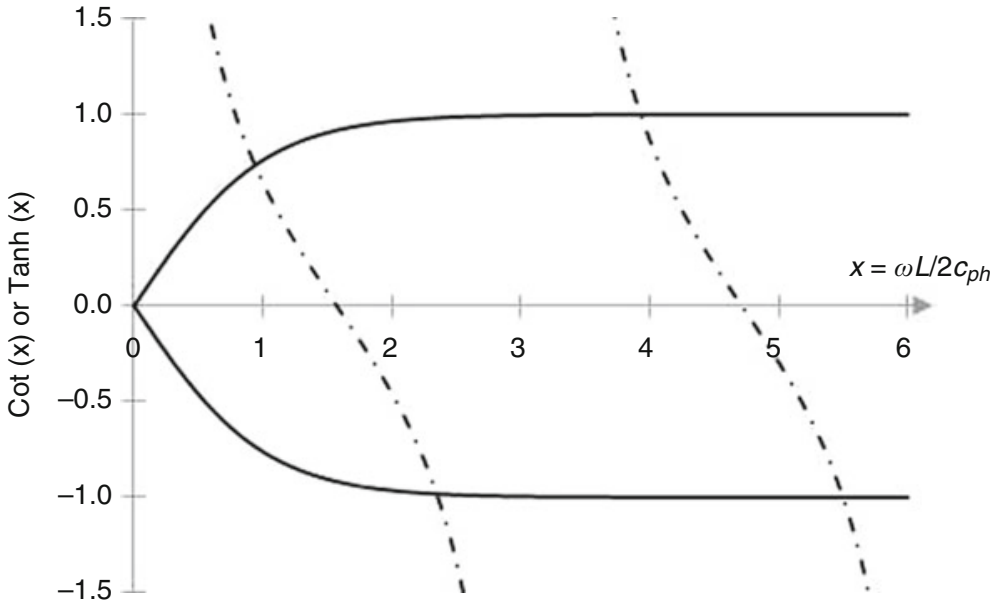

Eq. (5.6) or Eq. (5.29). Even with "ideal" boundary conditions, the normal mode frequencies of the flexing bar are not harmonically related. Also, for flexural modes, frequency is inversely proportional to the length of the bar squared. The frequencies also depend explicitly on the cross-sectional shape of the bar through the radius of gyration, $\kappa$, as defined in Eq. (4.26). Measurement of the modal frequencies and damping has been used to determine Young's modulus of small samples [13]. However, at least two independent modes have to be measured to determine both independent moduli for an isotropic solid [14].

\subsubsection{Flexural Standing Wave Mode Shapes}

The displacement amplitudes of the bar undergoing flexural vibrations in one of its normal modes can be determined by substituting the quantized wavenumber, $k_{n}=\omega_{n} / c_{p h}$, corresponding to the frequencies, $f_{n}$, of Eq. (5.50), into the mode shapes of Eq. (5.43).

$$
y(x)=A_{n}\left[\cosh \left(k_{n} x\right)-\cos \left(k_{n} x\right)\right]+B_{n}\left[\sinh \left(k_{n} x\right)-\sin \left(k_{n} x\right)\right]
$$

Following Eq. (5.45), $B_{n}$ can be expressed in terms of $A_{n}$.

$$
-B_{n}=A_{n} \frac{\cosh \left(k_{n} L\right)+\cos \left(k_{n} L\right)}{\sinh \left(k_{n} L\right)+\sin \left(k_{n} L\right)}=A_{n} \frac{\sinh \left(k_{n} L\right)-\sin \left(k_{n} L\right)}{\cosh \left(k_{n} L\right)+\cos \left(k_{n} L\right)}
$$

Since the amplitude is arbitrary until the initial conditions are specified, we can choose to normalize the amplitudes by requiring that the integral over the length of the bar of the squared amplitude be $(L / 2)$, just as it would be if the amplitude functions were just the sine and cosine functions used for standing wave solutions to the wave equation.

$$
\int_{0}^{L} y^{2}(x) d x=\frac{L}{2}
$$

Substitution of $k_{n} L$ into Eq. (5.52) with all $A_{n}=1 / \sqrt{2}$ makes $B_{1}=-0.518, B_{2}=-0.721$, and all subsequent $B_{n}=-1 / \sqrt{ } 2$ for $n \geq 3$.

The mode shapes for $1 \leq n \leq 4$ are shown in Fig. 5.8 and are compared to the solutions for the longitudinal modes of a fixed-free bar having the same wavelength. The ratio of the modal frequencies, 

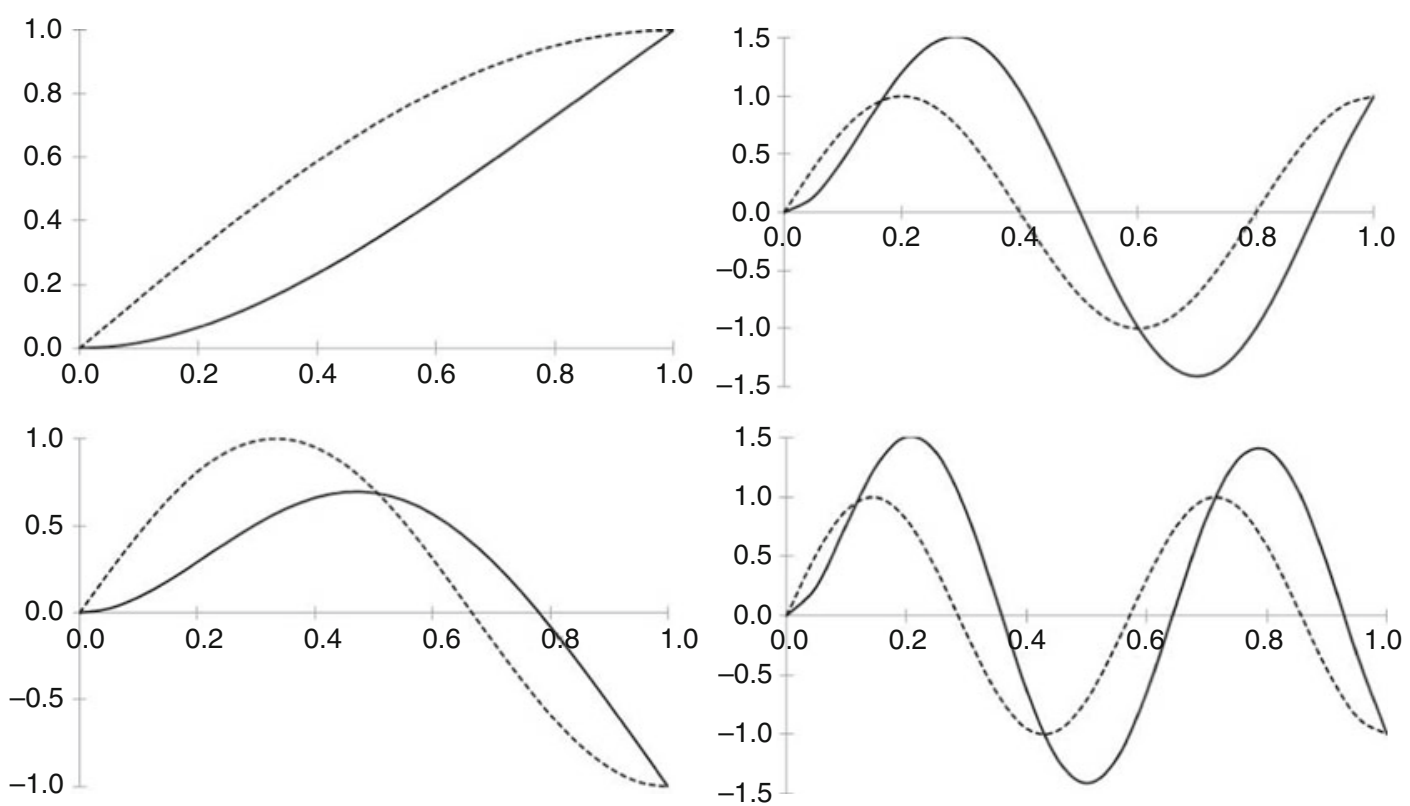

Fig. 5.8 The mode shapes for flexural vibration of clamped-free bars (solid line) are compared to the longitudinal mode shapes of a fixed-free bar having the same wavelength (dashed line) for the four lowest modes, normalized to match the displacements of both modes at $x=L$. Frequency ratios and the location of the zero-crossings are provided in Table 5.2

Table 5.2 Clamped-free (cantilevered) beam modal frequency ratios, $f_{n} / f_{1}$; normalized wavelengths, $\lambda_{n} / L$; and normalized location of standing wave displacement nodes as measured from the clamped end

\begin{tabular}{l|l|l|l|l|l}
\hline Mode & $f_{n} / f_{1}$ & $\lambda_{n} / L$ & First node $/ L$ & Second node $/ L$ & Third node $/ L$ \\
\hline 1 & 1.0000 & 3.3508 & & & \\
\hline 2 & 6.2669 & 1.3385 & 0.7806 & & \\
\hline 3 & 17.5475 & 0.7999 & 0.5017 & 0.8998 & \\
\hline 4 & 34.3863 & 0.5714 & 0.3584 & 0.6428 & 0.9286 \\
\hline
\end{tabular}

$f_{n}$, to the fundamental mode frequency, $f_{1}$, is provided in Table 5.2, along with the normalized wavelengths, $\lambda_{n} / L$, and the normalized location of the displacement nodes as measured from the clamped end.

A bar with clamped boundaries on both ends can be solved in the same way, starting with Eq. (5.43), which satisfies the boundary conditions at $x=0$. Using the same procedure to fit the clamped conditions at $x=L$, the wavenumbers are again quantized by a transcendental equation that is plotted in Fig. 5.9.

$$
\cosh \left(k_{n} L\right) \cos \left(k_{n} L\right)=1 \quad \text { or } \quad \tan \left(\frac{k_{n} L}{2}\right)= \pm \tanh \left(\frac{k_{n} L}{2}\right)
$$

The resulting values of $k_{n} L$ for the normal solutions of Eq. (5.54) can be combined with the phase speed in Eq. (5.39) to yield the flexural normal mode frequencies, $f_{n}$, of the clamped-clamped bar as a function of the geometry of the bar $(L$ and $\kappa)$ and the properties of the bar's material, $c_{B}=(E / \rho)^{1 / 2}$. 
Fig. 5.9 The two terms on the right-hand side of Eq. (5.54) are plotted as a function of $x=k L / 2=\omega_{n} L /$ $2 c_{p h}$. The hyperbolic tangents are shown as solid lines and the dashed lines are the tangent. Their intersections provide the normal mode frequencies, $\omega_{n}$, for a clamped-clamped or free-free bar

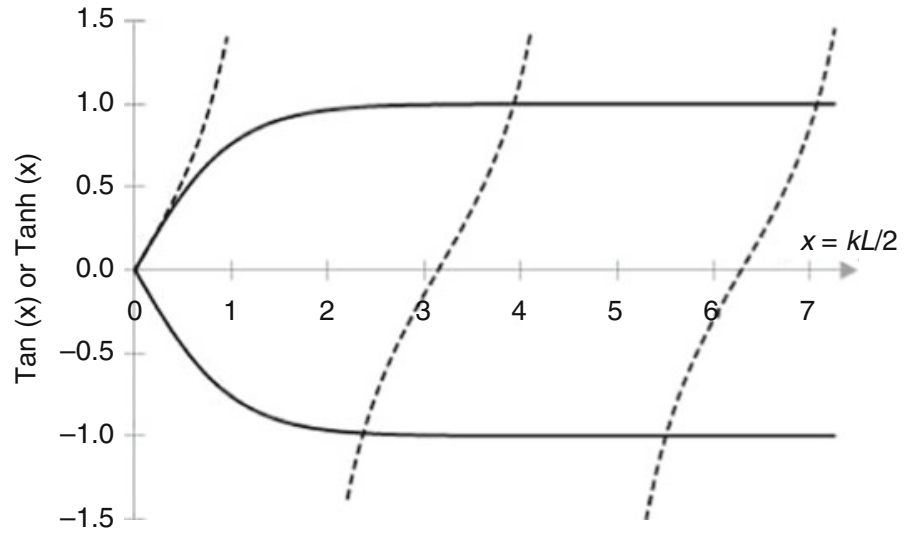

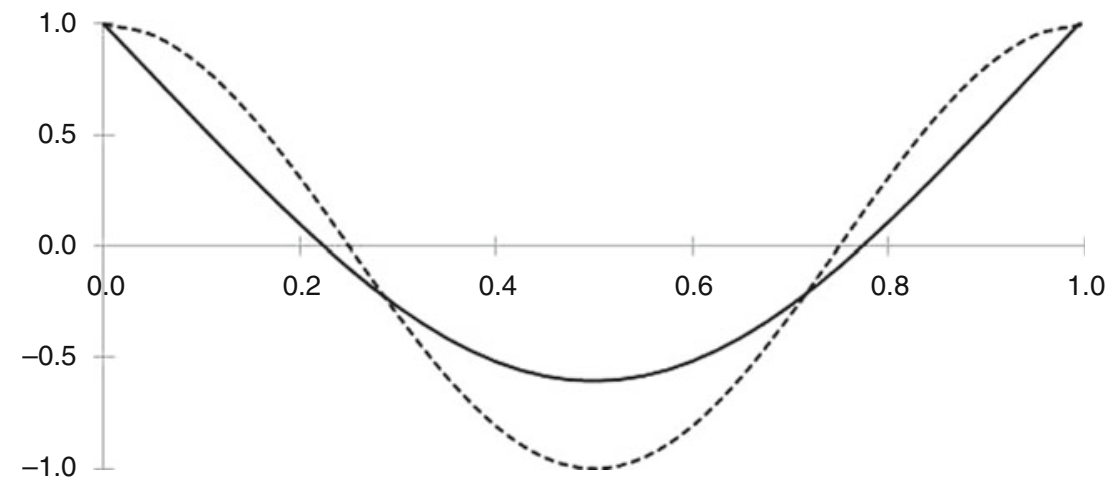

Fig. 5.10 The flexural mode shape for a free-free bar (solid line) is compared to the longitudinal mode shapes of a freefree bar (dashed line) for the fundamental vibrational mode. Normalized to match the displacements of the flexural mode at $x=0$ and $x=L$

$$
f_{n}=\frac{\omega_{n}}{2 \pi}=\frac{\pi}{8} \frac{c_{B} \kappa}{L^{2}}\left(3.01124^{2}, 4.99951^{2}, 7.00002^{2}, 9^{2}, 11^{2}, \ldots\right)
$$

The normal mode shapes are obtained, as before, by determining the $B_{n}$ 's, again assuming $A_{n}=1$ / $\sqrt{ } 2$.

$$
B_{n}=A_{n} \frac{\sin \left(k_{n} L\right)+\sinh \left(k_{n} L\right)}{\cos \left(k_{n} L\right)-\cosh \left(k_{n} L\right)}=-A_{n} \frac{\cos \left(k_{n} L\right)-\cosh \left(k_{n} L\right)}{\sin \left(k_{n} L\right)-\sinh \left(k_{n} L\right)}
$$

Substitution of $k_{n} L$ into Eq. (5.52) with all $A_{n}=1 / \sqrt{ } 2$ makes $B_{1}=-0.6947$, and all subsequent $B_{n}=-1 / \sqrt{2}$ for $n \geq 2$.

The flexural mode shapes for a free-free bar can be obtained by double differentiation with respect to $x$ of the clamped-clamped mode shapes, resulting in the rather surprising fact that the normal mode frequencies for both free-free and clamped-clamped bars in flexure are identical.

The fundamental mode shape for the $n=1$ free-free bar is shown in Fig. 5.10. Again, it is compared to the solutions for the fundamental longitudinal free-free mode of a thin bar having the same length. The ratio of the modal frequencies, $f_{n}$, to the fundamental mode frequency, $f_{1}$, is provided in Table 5.3. 
Table 5.3 Clamped-clamped or free-free bar modal frequency ratios, $f_{n} / f_{1}$, and normalized wavelengths, $\lambda_{n} / L$. The remaining columns to the right of $\lambda_{n} / L$ are the normalized location of standing wave displacement nodes for the freefree case

\begin{tabular}{|c|c|c|c|c|c|c|c|}
\hline Mode & $f_{n} / f_{1}$ & $\lambda_{n} / L$ & First node $/ L$ & Second node $/ L$ & Third/L & Fourth/L & Fifth $/ L$ \\
\hline 1 & 1.0000 & 1.3284 & 0.2244 & 0.7734 & & & \\
\hline 2 & 2.75654 & 0.8001 & 0.132 & 0.500 & 0.868 & & \\
\hline 3 & 5.40392 & 0.5714 & 0.094 & 0.356 & 0.644 & 0.906 & \\
\hline 4 & 8.93295 & 0.4444 & 0.073 & 0.277 & 0.500 & 0.723 & 0.927 \\
\hline
\end{tabular}
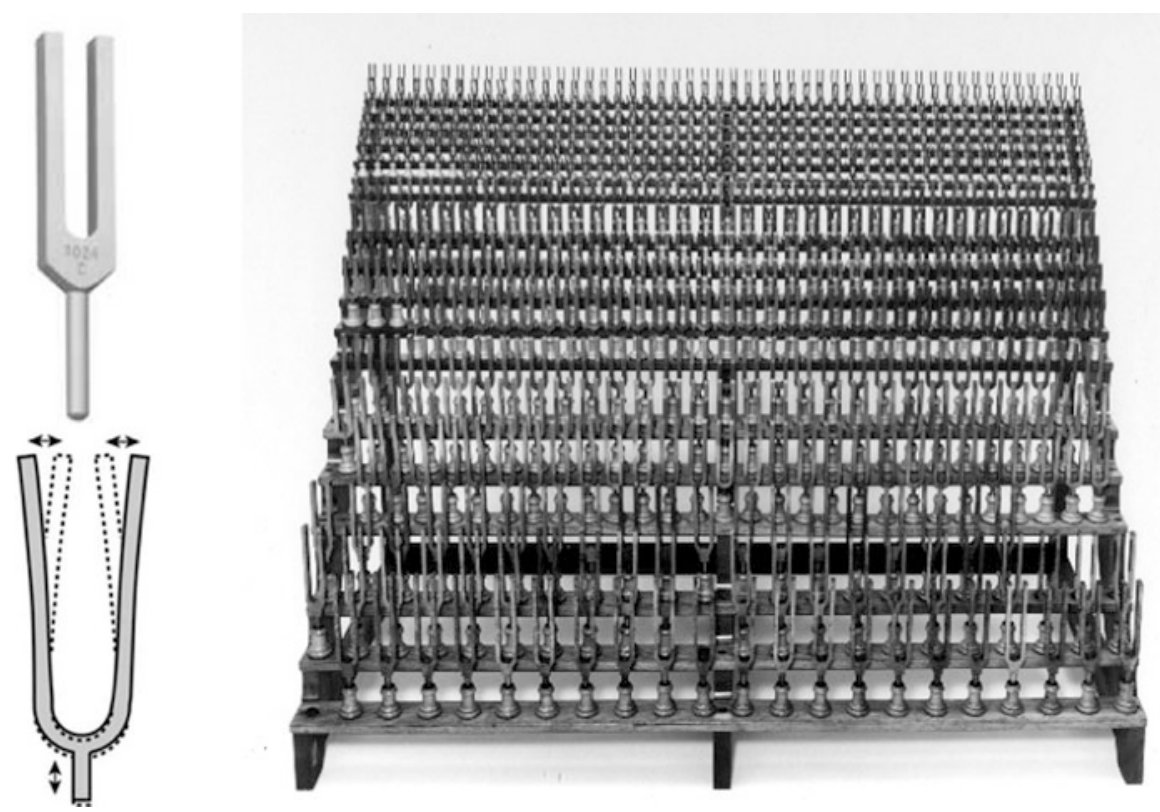

Fig. 5.11 (Left) Traditional tuning forks are shown above with its fundamental mode of vibration shown below. (Right) This "tonometer" contains 670 tuning forks ranging from $16 \mathrm{~Hz}$ to $4096 \mathrm{~Hz}$ (eight octaves) and was built by the Prussian physicist K. Rudolph Koenig (1832-1901). It was displayed at the London Exhibition, in 1852, where it was awarded the gold medal for scientific instruments and was transported to the Philadelphia Exposition where it appeared in 1876. (Photo courtesy of the Smithsonian National Museum of American History)

The free-free flexural modes of bars are the percussive tone generators in the glockenspiel and many children's toy instruments (e.g., Pixiphone). ${ }^{10}$ By supporting the resonant bars at the nodal locations for the fundamental mode (see Table 5.3), the higher overtones will be present when the bar is struck, providing those instruments with a characteristically dramatic attack, which decays quickly, leaving the fundamental mode to linger.

A very common configuration for a free-free bar vibrating in its flexural mode is the tuning fork, shown in Fig. 5.11 (left) along with the "tonometer" that was commonly used to check the pitch of musical instruments. You may use an electronic instrument to tune your guitar or musical instrument, but the frequency standard inside the electronic device is likely to be a tiny quartz tuning fork. In fact, if

\footnotetext{
${ }^{10}$ The glockenspiel has bars of uniform cross-section, so the ratio of their overtones to the fundamental is given in Table 5.3. The underside of the bars in a xylophone is thinned near their center to make their overtones the ratio of integers: $f_{2} / f_{1}=3$ and $f_{3} / f_{1}=6$.
} 
Fig. 5.12 Distortion of the free surface of a solid due to the propagation of a Rayleigh wave. Arrows indicate the motion of the material due to the wave

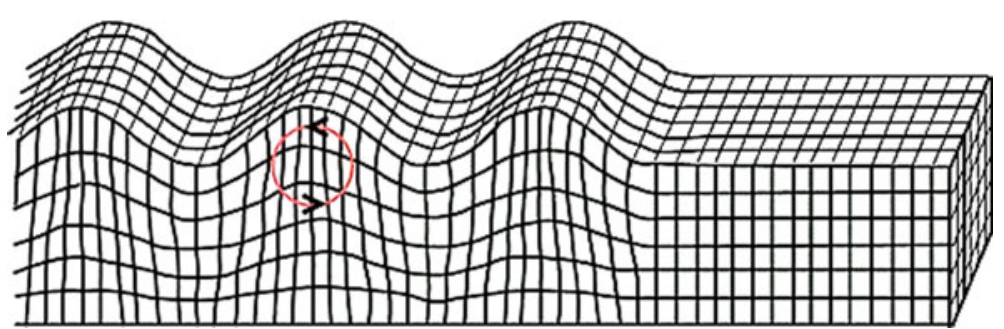

you own a "smartphone," it probably has a tuning fork with tines that are about $2 \mathrm{~mm}$ long that is used as a gyroscope (angular rate sensor). The same is true for games, automotive anti-skid and navigation sensors, remote-controlled aircraft (drones), digital cameras, etc.

When the tines of a tuning fork are oscillating, as shown in Fig. 5.11, and the entire fork is rotated with some angular velocity, $\vec{\Omega}$, then the Coriolis force [15] can be expressed as $\vec{F}=2 M(\vec{v} \times \vec{\Omega})$, where $M$ is the effective moving mass of the vibrating tines and $\vec{v}$ is the velocity of the tines. This force will cause the tines to develop a component of their motion in a plane that is orthogonal (due to the cross-product) to the original plane of vibration. This out-of-plane motion is then sensed to determine the rate of rotation [16].

\subsubsection{Rayleigh Waves*}

In principle, the phase speed in Eq. (5.39) could exceed the speed of light for large enough values of frequency, $\omega$. At such frequencies, the assumption that the transverse dimensions of the bar are much smaller than the wavelength $\left(S^{1 / 2} \ll \lambda\right)$ would be violated. As the frequency increases, these flexural waves do not bend the entire bar but become localized deformations at the surface. Such waves are known as Rayleigh waves [17], and their associated deformations are diagrammed in Fig. 5.12. A derivation of their propagation speed would take us too far from our development of waves on bars and is available elsewhere [7]. The speed of Rayleigh waves is about $10 \%$ slower than the shear wave speed, $c_{S}$, and is nondispersive.

The Rayleigh wave solutions are not contained in Eq. (5.36) because that equation does not include the contributions of rotary inertia and shear that become important at higher frequencies [18].

\subsection{Resonant Determination of Elastic Moduli}

\footnotetext{
"One of the most precise ways of measuring the elastic constants of a substance is by measuring the density of the material and the speeds of two kinds of waves." R. P. Feynman. [14]
}

Having derived the normal mode frequencies for longitudinal, torsional, and flexural vibrations of a thin bar, this section will describe a simple and inexpensive apparatus that can selectively excite and detect these modes. In addition to the pedagogical value of such an apparatus, it is also an excellent way to determine the elastic moduli of materials that are not ferromagnetic [19]. Sample data will be presented and analyzed for a composite of high tensile strength epoxy [20] with 20\% glass fibers (by weight) to determine its Young's and shear moduli. In Sect. 5.4.5, the technique will be used to illustrate the viscoelastic behavior of an elastomer.

The (intentionally crude) apparatus, shown in Fig. 5.13, suspends the ends of the bar sample in between the pole pieces of two strong horseshoe magnets. Both ends of the bar are free and have coils 
Fig. 5.13 Photograph of an apparatus for selective excitation and detection of longitudinal, torsional, and flexural resonance of a thin bar. The bar is supported by crossed rubber bands attached to ring stands that suspend the bar with its ends in the gap between "horseshoe" magnets. The sample in the photo is about $25 \mathrm{~cm}$ long and $1.2 \mathrm{~cm}$ in diameter

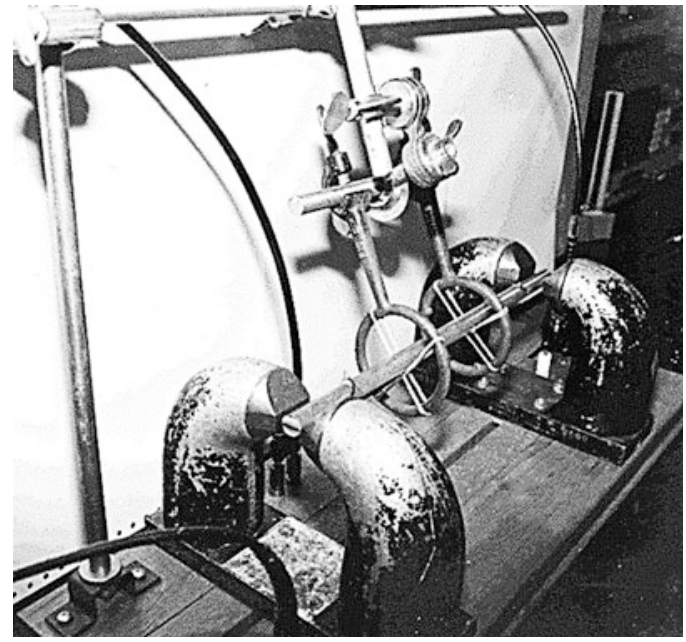

of wire glued to their free ends that are used as the electrodynamic transducers for all three modes. In this apparatus, the gap between the magnets' pole pieces is $2.0 \mathrm{~cm}$ and the magnitude of the magnetic induction, $|\vec{B}|$, at the center of the gap between the pole pieces is about 2.0 kilogauss $=0.20$ tesla.

The transducers are just two coils (about ten turns each) of ordinary insulated solid copper magnet wire that were wound around the handle of a screwdriver. ${ }^{11}$ \#28 gauge copper wire (376 micron diameter) has a current-carrying capacity of about $300 \mathrm{~mA}$, which is more than adequate for the driven coil. ${ }^{12}$ It is convenient to leave about $50 \mathrm{~cm}$ of wire at both ends of each coil that can be used to attach the coil to the drive amplifier or the measurement system (e.g., low-noise pre-amplifier, ${ }^{13}$ oscilloscope, spectrum analyzer, or typically, all three).

Before attaching the coils, the bar should be weighed and its physical dimensions determined as accurately as possible. Those measurements will be used to determine the mass density of the sample. After weighing, the coils can be attached to the ends of the sample using any appropriate adhesive. Care must be taken to be sure that the plane defined by both coils is aligned, since the magnets' fields are usually both in the same direction. For most samples, a simple glue, like that used to assembly model airplanes (e.g., Duco ${ }^{\mathrm{TM}}$ cement), or even clear nail polish, ${ }^{14}$ is entirely adequate since the forces the drive coil will be modest, typically less than $20 \mathrm{mN}$. After the coils have been glued to the sample, the assembly should be weighed again so that an effective length correction can be added to the physical length of the sample to correct for the mass of the coils and their adhesive added to the ends of the bar (see Sect. 5.4.4).

\footnotetext{
${ }^{11}$ A screwdriver handle makes an ideal mandrel since the handle has grooves to improve grip that provide spaces to weave the last turn in and out of the grooves to hold the coil together when it is slipped off the screwdriver's handle.

${ }^{12}$ Almost no current flows through the detection coil, so it could be much smaller gauge wire, but it is often convenient to make both coils from the same wire.

${ }^{13}$ If a low-noise voltage pre-amplifier is available (e.g., PAR 113, Ithaco 1201, or SRS 560), they usually also provide some adjustable low-pass filtering capabilities that can remove low-frequency seismic vibrations if the apparatus is on a table that is not rigid.

${ }^{14}$ Clear nail polish has lower viscosity, making attachment of the coils easier since capillarity will draw the polish into the coil and concentrate where the coil contacts the bar.
} 


\subsubsection{Mode-Selective Electrodynamic Excitation and Detection}

The torsional mode can be excited preferentially by orienting both coils horizontally with the pole pieces also horizontally, as shown in Fig. 5.14 (top right). This technique was first used by Barone and Giacomini [21] to study the modes of bars having variable cross-section and then by Leonard [22] who measured the attenuation of torsional modes to disprove the existence of the "Fitzgerald effect." $[23,24]^{15}$ The short portion of the coil, which crosses the end of the bar along a diameter, should stick out slightly beyond the pole pieces. When the alternating electrical current, $I(t)=i_{1} \cos (\omega t)$, flows in one direction through the drive coil, a Lorenz force, $|\vec{F}(t)|=(B \ell) I(t)$, will be downward on one side of the coil and upward on the other, where $\ell$ is the total length of wire in the coil subjected to the magnetic field on one side of the bar. When the current changes direction, so will the forces. This twisting moment will selectively excite the torsional modes of the bar.

The other coil, on the opposite end of the bar, is in the same orientation with respect to its magnet and will generate a voltage (emf) that is proportional to the time rate of change of the magnetic flux, $\Phi$,
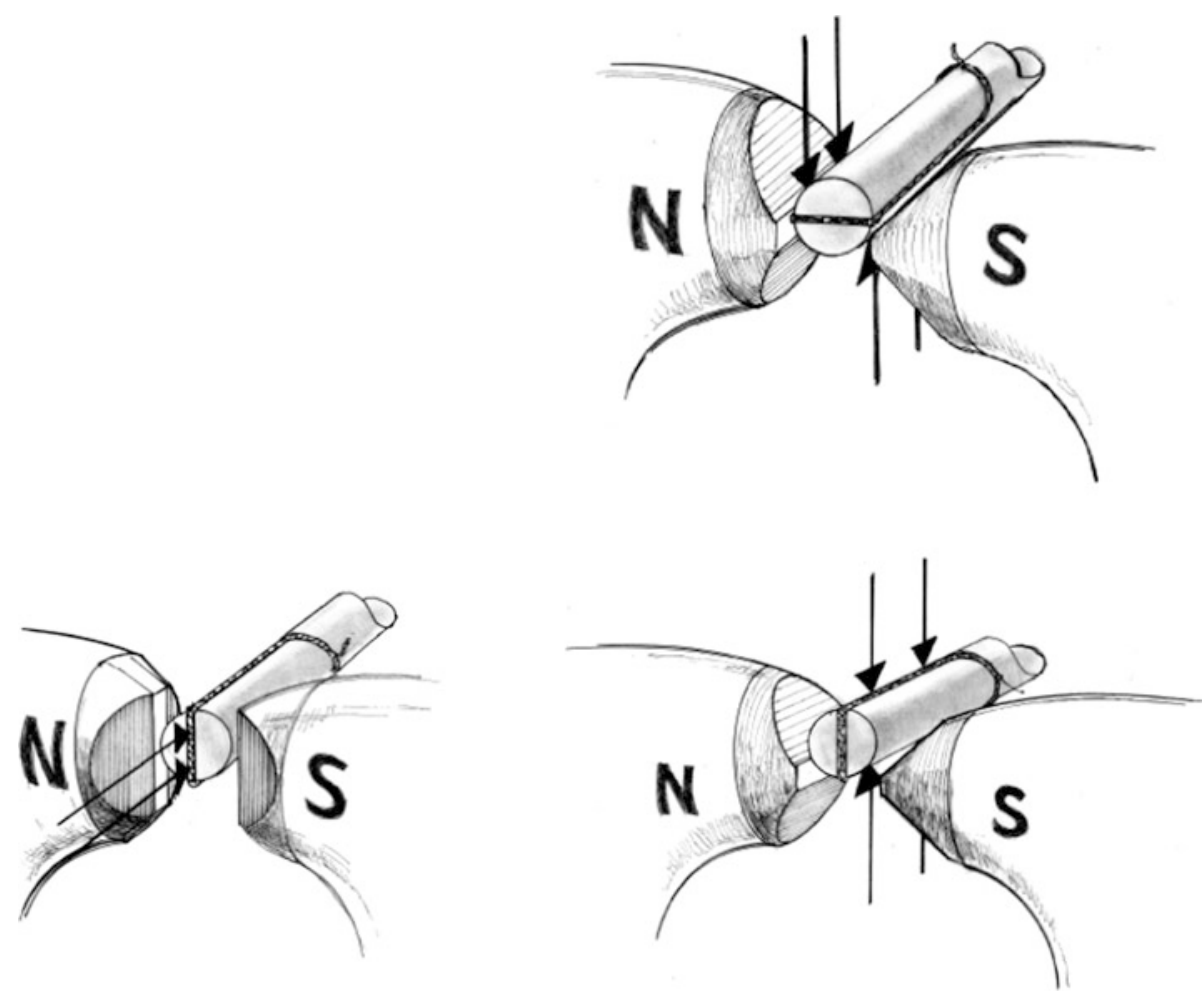

Fig. 5.14 Arrangements of the electrodynamic transducer coils in the magnet gap for excitation and detection of the torsional (top right), flexural (bottom right), and longitudinal (bottom left) modes of a thin bar. The arrows show the strength and direction of the forces produced by the current flowing through the coils that are functioning as the drive transducers

\footnotetext{
${ }^{15}$ Fitzgerald had thought he discovered a new attenuation mechanism, but Leonard showed that Fitzgerald's "effect" was absent, and Fitzgerald had just measured an artifact of the attachment of piezoelectric transducers to his sample.
} 
through the receiver coil. The flux, $\Phi$, is the product of the projection of the surface area of the coil, $A_{\perp}=\mathrm{A} \cos \phi$, times the magnitude of the magnetic induction, $|\vec{B}|$, times the number of turns or wire, $N$. Here $A$ is the area within the coil, and $\phi$ is the angle between a vector normal to the plane of the coil and the magnetic field lines.

$$
e m f=-\frac{d \Phi}{d t}=-N|\vec{B}| \frac{d A_{\perp}}{d t}=-N|\vec{B}| A \frac{d(\cos \phi)}{d t}
$$

With the coil oriented as shown in Fig. 5.14 (top right), $\phi \cong 90^{\circ}$, so $A_{\perp} \cong 0$, and $\Phi \cong 0$. When a torsional wave is excited, the end of the bar will experience a time-harmonic twisting, so $\phi(t)=\mathfrak{R} e$ $\left[\phi_{1} e^{j \omega t}\right]$, generating a voltage (emf) given by Faraday's law in Eq. (5.57). With $\phi \cong 90^{\circ}$, the sensitivity of the transducer to the twisting will be its greatest. The emf will be generated at the same frequency, $\omega$, as the twisting that is caused by the torsional resonance and will also be proportional to the amplitude, $\phi_{1}$.

Flexural resonances can be excited and detected by simply rotating the bar (with its attached coils) by $90^{\circ}$ from the torsional mode orientation and raising (or lowering) the bar by one bar radius. This places one portion of each coil in the strongest part of the magnetic field and lifts the other part of the coil to a region of weaker magnetic field, as shown in Fig. 5.14 (bottom right). Now the vertical forces on each long section of the coil will be in opposite directions. Because of the stronger magnetic field is applied to the lower portion of the coil (as shown), there will be a net vertical force that will create a harmonic bending of the bar and excite the flexural modes of vibration.

In this orientation, the coil on the opposite end of the bar will be "dipping" in and out of the stronger regions of magnetic field. In this case $\phi \cong 0^{\circ}$, so $A_{\perp} \cong A$, but the magnitude of the magnetic induction, $|\vec{B}|$, will be changing with time, $B(t)=B_{o}+\mathfrak{R} e\left[B_{1} e^{j \omega t}\right]$, as the receiver coil moves toward and away from the strongest regions of magnetic field. Now Eq. (5.57) will produce an emf $=N A(\mathrm{~d} B / \mathrm{d} t)$ that will be at the same frequency as the flexural vibrations and proportional to their amplitudes.

Finally, the longitudinal modes can be selectively excited and detected by reorienting the pole pieces to make the gap vertical, as shown in Fig. 5.14 (bottom left). This concentrates the magnetic field in the vertical direction. The end of the coil that is along the diameter at the end of the bar is placed in the strongest portion of that magnetic field. In this orientation, the driven end of the coil will be putting oscillatory longitudinal forces on the end of the bar thus exciting the longitudinal modes. Since the length of that portion of the coil is considerably shorter than the portions of the coil along the perimeter of the sample, the longitudinal modes will not be excited as strongly as the torsional and flexural modes for the same electrical current amplitude, $i_{l}$, flowing through the driving coil. Detection of the longitudinal mode is the same as the flexural mode. The magnetic flux, $\Phi(t)$, is modulated by the coil's motion, being pushed into and out of the strongest portions of the magnetic field by the motion of the end, $\boldsymbol{\xi}(t)=\left[\widehat{\xi}^{j \omega t}\right]$.

\subsubsection{Bar Sample Size and Preparation}

Sample data acquired by this method are displayed in Fig. 5.16 for the epoxy-glass composite described at the beginning of Sect. 5.4. The bar was fabricated by mixing the epoxy and then adding glass fibers to the mix. The mixture was then poured into a hollow plastic tube that had been coated on the inside with a mold release agent to facilitate removal of the sample after the epoxy had cured. The sample was pushed out of the tube and the ends were cut straight using a band saw.

Before the transducer coils were attached, the bar was weighed, $M=55.723 \pm 0.001 \mathrm{~g}$, and the bar's dimensions were measured. The length $L=33.60 \pm 0.02 \mathrm{~cm}$, but the cross-section was found to 
be slightly elliptical with major and minor diameters ranging from $d_{\max }=13.17 \pm 0.02 \mathrm{~mm}$ to $d_{\text {min }}=12.77 \pm 0.20 \mathrm{~mm}$, yielding an average diameter of $d=12.97 \pm 0.20 \mathrm{~mm}$. These physical measurements produced an average mass density, $\rho=1256 \pm 4 \mathrm{~kg} / \mathrm{m}^{3}$. It is not unusual to have this degree of ellipticity in a sample which was cast in a plastic tube. The cross-section of glass tubes is more circular, but also more dangerous to fabricate, since the sample can seldom be removed without breaking the glass.

The transducer coils were attached as described previously using Duco ${ }^{\mathrm{TM}}$ cement. With a bar that has an elliptical cross-section, it is preferable to orient the coils along either the major or minor diameter so that the polarization of the flexural mode is known. For this sample, the major and minor diameters differed by about $3 \%$ corresponding to a $6 \%$ difference in the free-free flexural mode frequencies caused by the difference in the $\kappa^{2}$ value that appears in Eq. (5.55).

After attachment of the coils, the bar was weighed again and found to be $1.5 \pm 0.1 \mathrm{~g}$ heavier. It is difficult to be sure exactly how much the motion of the wires that connect the coil to the instrumentation contribute to the measurement, but in this case, the accuracy is not degraded significantly since the uncertainty in the effective length of the bar (see Sect. 5.4.4) is only about $1 / 2 \%$.

\subsubsection{Measured Resonance Spectra}

Figure 5.16 displays three resonance spectra that were acquired using a dynamic signal analyzer to measure the bar's response only in a narrow band around the frequency at which it was being driven. The setup is diagrammed schematically for the torsional mode in Fig. 5.14 (top right). The analyzer also had an internal curve-fitting program, so the measured spectral response is shown as the solid line and the curve-fit to that response is shown as the dashed line (Fig. 5.16).

The five lowest-frequency torsional modes are shown in Fig. 5.16 (upper right). They appear to be harmonically related, with uniform spacing between the resonances. That appearance is confirmed by the resonance frequencies listed in Table 5.4. It is also worthwhile to note that the dynamic range (the ratio of the largest measured signal to the smallest) of that measurement is about $70 \mathrm{~dB}$, corresponding to an amplitude ratio in excess of 3000:1. At the minima in the spectrum (i.e., at frequencies between the resonances), the curve-fit dashed line alternates between being larger and smaller than the measurements. That is because the coils also act as an electrical transformer coupling the oscillating magnetic flux in the drive coil (weakly) into the receiver coil. This could be eliminated by orienting the planes of the two coils to be mutually perpendicular and rotating one set of magnets by $90^{\circ}$ or by placing a magnetic shield (iron is good, $\mu$-metal is better) around the middle of the bar without

Fig. 5.15 Schematic representation of the connection of the dualchannel spectrum analyzer that measured the transfer function between the drive signals, monitored in Channel 1, and the bar's resonance responses monitored in Channel 2. As shown, the apparatus was set up to measure the torsional modes of the bar [25]

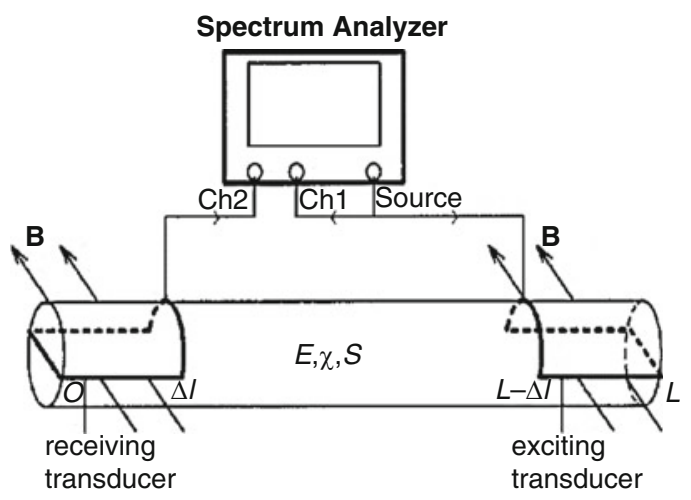



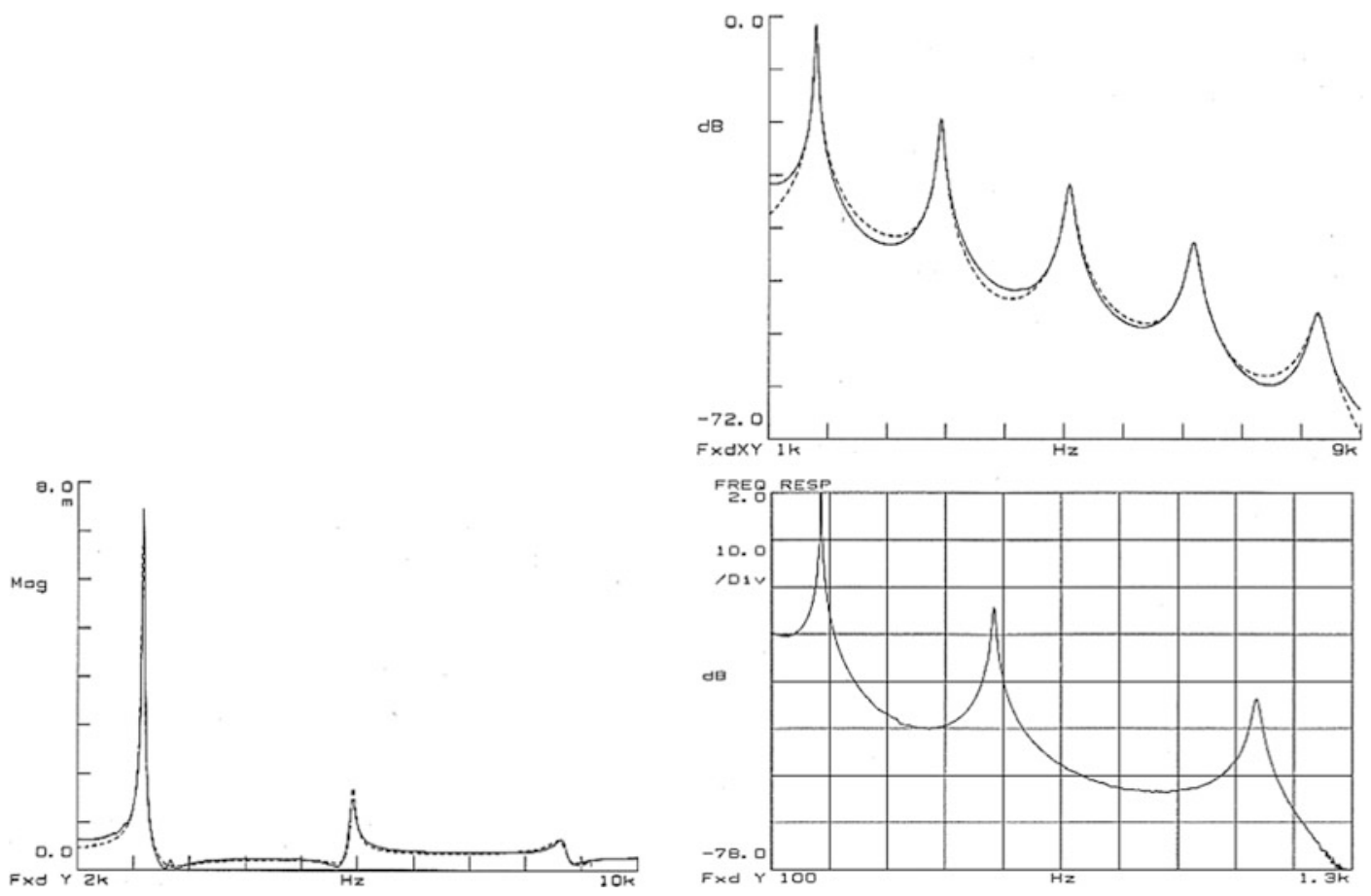

Fig. 5.16 Resonance spectra for the lowest-frequency torsional (upper right), flexural (lower right), and longitudinal (lower left) resonances. The solid lines are the measured spectra, and the dashed lines were pole-zero fits to those measured spectra

Table 5.4 Summary of the measured normal mode frequencies of the thin bar sample of an isotropic glass-epoxy composite. The error is the standard deviation of the average of the normalized frequencies, $f_{n} / f_{1}$, for each mode

\begin{tabular}{|c|c|c|c|}
\hline Mode number $[n]$ & Frequency $[\mathrm{Hz}]$ & Normalized frequency $\left[f_{n} / n\right]$ & Average uncertainty $[\mathrm{Hz}(\%)]$ \\
\hline \multicolumn{4}{|l|}{ Torsional } \\
\hline 1 & 1644 & 1644 & \\
\hline 2 & 3341 & 1670 & \\
\hline 3 & 5080 & 1693 & \\
\hline 4 & 6750 & 1688 & \\
\hline 5 & 8446 & 1689 & \\
\hline Average & & 1677 & $\pm 20( \pm 1.2 \%)$ \\
\hline \multicolumn{4}{|l|}{ Flexural } \\
\hline$(3.01124)^{2}$ & 203.1 & 22.40 & \\
\hline$(4.99951)^{2}$ & 560.4 & 22.42 & \\
\hline$(7.00002)^{2}$ & 1104 & 22.53 & \\
\hline Average & & 22.45 & $\pm 0.07( \pm 0.2 \%)$ \\
\hline \multicolumn{4}{|l|}{ Longitudinal } \\
\hline 1 & 2941 & 2941 & \\
\hline 2 & 5936 & 2968 & \\
\hline 3 & 8896 & 2965 & \\
\hline Average & & 2958 & $\pm 15( \pm 0.4 \%)$ \\
\hline
\end{tabular}


touching the bar. Those methods of eliminating the electromagnetic cross talk between the drive and receiver coils were not implemented because the signal-to-noise ratio was more than adequate to accurately determine the peak frequencies that were not measurably influenced by the electromagnetic cross talk.

Table 5.4 lists the measured resonance frequencies of the five lowest-frequency torsional modes. Since the modes are expected to be related harmonically, the table also forms the ratio between the frequencies, $f_{n}$, of the $n^{\text {th }}$ mode, to the fundamental frequency, $f_{l}$. For harmonic modes of a free-free bar, that ratio should be a constant. The ratio of the standard deviation of the five modal frequencies to their average is $\pm 1.2 \%$. Since this is in the range of our relative uncertainty of the mass density $( \pm 0.3 \%)$, and the ellipticity $( \pm 3 \%)$, there was no motivation to improve or to understand the source of that small anharmonicity.

The three lowest-frequency flexural modes of the bar are shown in the spectrum in Fig. 5.16 (lower right). It is obvious from inspection of that spectrum that those modes are not harmonically related. The frequency difference between the first and second modes is smaller than the difference between the second and third, as would be expected from Eq. (5.55). It is also possible to see a small amount of noise in the spectrum that appears in the "spectral tail" above $1.2 \mathrm{kHz}$. The dynamic range of that spectrum is $80 \mathrm{~dB}$, corresponding to an amplitude ratio of 10,000:1. The frequencies of those modes are listed in Table 5.4, as is their normalized frequency, $f_{n} / f_{l}$, which has a relative uncertainty of $\pm 0.2 \%$.

Since the shear modulus can be related to the torsional mode frequencies and Young's modulus related to the flexural mode frequencies, we have enough information to determine the complete elastic response of our sample that is assumed to be isotropic. ${ }^{16}$ Since the longitudinal modes were also measured, they can be used to estimate the accuracy of the method for the determination of Young's modulus.

The spectrum displaying those longitudinal modes is shown in Fig. 5.16 (lower left). As mentioned, the amplitudes of the longitudinal modes are smaller than of the other two modes because the transduction is less efficient. The small bump to the right of the largest longitudinal mode is a weak excitation of the second torsional mode at $3341 \mathrm{~Hz}$. Since the side portions of the coils are so much longer than the short section of coil at the end, it is possible to excite a small amount of torsional vibration even though the magnets and coils are arranged to couple preferentially to the longitudinal mode. The third resonance at nearly $9 \mathrm{kHz}$ hardly looks like a resonance at all, again due to phase cancellation between the superposition of the acoustical resonance signal and the electromagnetic cross talk signal. Despite those limitations, the relative uncertainty of the normalized longitudinal mode resonance frequencies in Table 5.4 is only $\pm 0.4 \%$, which is consistent with the precision of the measurements of the frequencies of the other two modes.

The epoxy-glass composite used in this example was chosen because it does not have a particularly large quality factor, $Q$, making measurement of its modal frequencies a bit more challenging. Wooden bars will produce similar results. If this same apparatus is used to measure the resonances of metal bars (e.g., aluminum or brass) or ceramics (e.g., alumina) or glass, the $Q$ will be significantly larger, as will be the signal-to-noise ratio. Consequently, the results for the moduli will be even more precise.

\footnotetext{
${ }^{16}$ Although this sample is a composite, the glass fibers in the epoxy matrix are short (about 800 microns long) and randomly oriented. The sample behaves isotropically upon length scales that are on the order of the diameter and the resonance wavelengths.
} 


\subsubsection{Effective Length Correction for Transducer Mass}

If any addition to a bar be made at the end, the period of vibration is prolonged. J. W. Strutt (Lord Rayleigh). [26]

Before calculating the moduli from the experimental data, we can eliminate one source of systematic error. Because the coils and their adhesives add mass to the end of the bar, we can calculate an effective length correction to account for this, just as was done for the quartz crystal microbalance in Sect. 5.1.2, except this time we will use Rayleigh's method instead of an approximation to the transcendental equation diagrammed in Fig. 5.2. Such effective length corrections were calculated by Rayleigh in Theory of Sound for the free-free longitudinal case in Vol. I, §155. The free-free flexural case is covered in Vol. I, §186. Rayleigh did not do the calculation for mass loading of the free-free torsional modes [26].

The mass of the transducers is concentrated very close to the free ends of the bar, and the length of the coils is much shorter than the length of the bar, so we can assume that all of the mass of the coil is concentrated at the end, at least for the lower-frequency modes, where the wavelength of the resonance exceeds the length of the bar or is a substantial fraction of the bar's length. We will ignore the stiffness added by the coils since they are attached close to the stress-free ends. ${ }^{17}$ The coils only affect the bar's mass, so we can apply Rayleigh's method (see Sect. 3.2.2) and only concern ourselves with the changes in the kinetic energy, $\delta(K E)$, that cause changes in frequency, $\delta \omega=2 \pi \delta f$, due to the mass loading of the coils. Since $\omega^{2} \propto(P E / K E)$, the torsional and longitudinal modal frequencies are inversely proportional to the length of the bar, and it is possible to express the length correction, $\delta L$, in terms of the change in kinetic energy, $\delta(K E)$.

$$
\frac{\delta f}{f}=\frac{\delta \omega}{\omega}=-\frac{\delta L}{L}=-\frac{1}{2}\left(\frac{\delta K E}{K E}\right) .
$$

The effective length, $L_{\text {eff }}$, of the bar that compensates for the mass loading of the coils is related to the original length, $L: L_{e f f}=L+\delta L$. If the mass of the bar prior to the attachment of the coils is $M$ and the mass added by the coils is $2 m$, then after the coils are attached, $M_{t o t}=M+2 m \equiv M+\Delta M$, where $(\Delta M) g$ is the change in the weight of the bar after the transducer coils are attached.

The calculation for the effective length of the bar, in the lowest-frequency longitudinal mode, is simplified if the symmetry of that mode is exploited. Since the center of the bar is a node, the entire lefthalf of the bar can be replaced by a rigid (fixed) end at $x=0$. The fundamental longitudinal resonance can be treated as the fundamental resonance of a fixed-free bar of length, $L^{\prime}=L / 2$. The displacements, $\xi(x, t)$, of the fixed-free half-bar can be written in terms of the maximum displacement amplitude, $\xi_{1}$, of the free end, with $\omega / k=c_{B}$ and $k_{1}$ given by Eq. (5.14).

$$
\xi(x, t)=\xi_{1} \sin \left(\pi \frac{x}{2 L}\right) \sin (\omega t)
$$

The kinetic energy of the half-bar undergoing longitudinal oscillations, $\left(K E_{1}{ }^{L} / 2\right)$, without the added mass, can be determined by integration along the length of the half-bar, $L^{\prime}=L / 2$, where the half-mass of the half-bar is $M^{\prime}=M / 2$.

\footnotetext{
${ }^{17}$ The stiffness contributions of the transducer coils have been calculated by Guo and Brown [25].
} 


$$
\frac{K E_{1}^{L}}{2}=\frac{\rho S \omega^{2} \xi_{1}^{2}}{2} \int_{0}^{L \prime} \sin ^{2}\left(\pi \frac{x}{2 L}\right) d x=\frac{1}{4} M^{\prime} \omega^{2} \xi_{1}^{2}
$$

The one coil of mass, $m$, at the end of the bar, will move with the velocity of the end of the bar, $\omega \xi_{o}$ $\sin \omega t$, and will have a maximum kinetic energy, $K E_{\text {coil }}=(m / 2) \omega^{2} x_{1}^{2}$. The relative shift in the kinetic energy of the half-bar will be $\delta(K E) /(K E)=K E_{\text {coil }} /\left(K E_{o}{ }^{L} / 2\right)=2 m / M$.

$$
\left(\frac{\delta L}{L}\right)_{L}=-\frac{\delta f}{f}=\frac{1}{2} \frac{K E_{\text {coil }}}{\left(K E_{o}^{L} / 2\right)}=\frac{m}{M}=\frac{2 m}{M}=\frac{\Delta M}{M} \Rightarrow L_{\text {eff }}^{L}=L\left(1+\frac{\Delta M}{M}\right)
$$

The same style of argument can be applied to the torsional case, except that the location of the added mass with respect to the axis of the bar is important because it is the moment of inertia, $I$, of the coil that will load the bar's torsional oscillations. For this case, the kinetic energy is due to rotation, so that $K E_{\text {coil }}=(1 / 2) I_{\text {coil }}\left(\omega \theta_{1}\right)^{2}$, where $\theta_{1}$ is the maximum angular displacement at the end of the bar in the fundamental mode. The moment of inertia of a disk of mass, $\delta m$, and diameter, $d$, is $I=(1 / 8)(\delta m) d^{2}$, so the kinetic energy of torsional oscillations, $\left(K E_{l}{ }^{T} / 2\right)$, of the half-bar is again given by integration over the half-length of the bar, $L^{\prime}$.

$$
\frac{K E_{1}^{T}}{2}=\frac{\rho S \omega^{2} \theta_{1}^{2}}{2} \frac{d^{2}}{8} \int \sin ^{2}\left(\pi \frac{x}{2 L}\right) d x=\frac{M^{\prime} \omega^{2} \theta_{1}^{2} d^{2}}{32}
$$

For an ellipse, $d^{2}=d_{\max } d_{\text {min }}$.

Since the single coil mass, $m$, is primarily located at the bar's radius, its moment of inertia is $I_{\text {coil }}=(1 / 4) m d^{2},{ }^{18}$ and the change in the kinetic energy of rotation caused by the addition of one coil to the end of the half-bar is $K E_{\text {coil }}^{T}=(1 / 8) m\left(d \omega \theta_{1}\right)^{2}$.

$$
\left(\frac{\delta L}{L}\right)_{T}=-\frac{\delta f}{f}=\frac{1}{2} \frac{K E_{\text {coil }}}{\left(K E_{o}^{T} / 2\right)}=\frac{2 m}{M^{\prime}}=\frac{4 m}{M}=\frac{2 \Delta M}{M} \Rightarrow L_{\text {eff }}^{T}=L\left(1+\frac{2 \Delta M}{M}\right)
$$

The mass of the coils have twice the effect on the frequency of the torsional modes, hence producing twice the effective length correction, $L_{\text {eff }}^{T}$, as that required for the longitudinal modes, $L_{e f f}{ }^{L}$, in Eq. (5.61).

The exact solution for the fundamental flexural mode of a free-free bar is again given by the superposition of four trigonometric functions with $k_{1} L=4.73, A_{1}=0.5$, and $B_{1}=-0.982 A_{1}$.

$$
y(x, t)=A_{1}\left[\cosh \left(k_{1} x\right)+\cos \left(k_{1} x\right)\right]+B_{1}\left[\sinh \left(k_{1} x\right)+\sin \left(k_{1} x\right)\right] \sin \left(\omega_{1} t\right)
$$

The kinetic energy of the bar without coils, vibrating in its flexural mode, $K E_{l}{ }^{F}$, is related to the integral of its transverse velocity, $(\partial y / \partial t)$, over its length.

$$
K E_{1}^{F}=\frac{\rho S}{2} \int_{0}^{L}\left(\frac{\partial y}{\partial t}\right)^{2} d x
$$

Rather than solving that integral for the kinetic energy of the unloaded bar in its fundamental mode using Eq. (5.64), we can create a second-order polynomial function, $y_{\text {poly }}(x, t)$, to approximate the

\footnotetext{
${ }^{18}$ Since the coil is actually on the surface of the bar, its diameter is slightly larger than $d$. We will neglect this difference by arguing that the part of the coil that crosses the bar's end has a lower moment of inertia. It would be a small correction to an already small correction (so works the rationalizations in the mind of an experimentalist).
} 


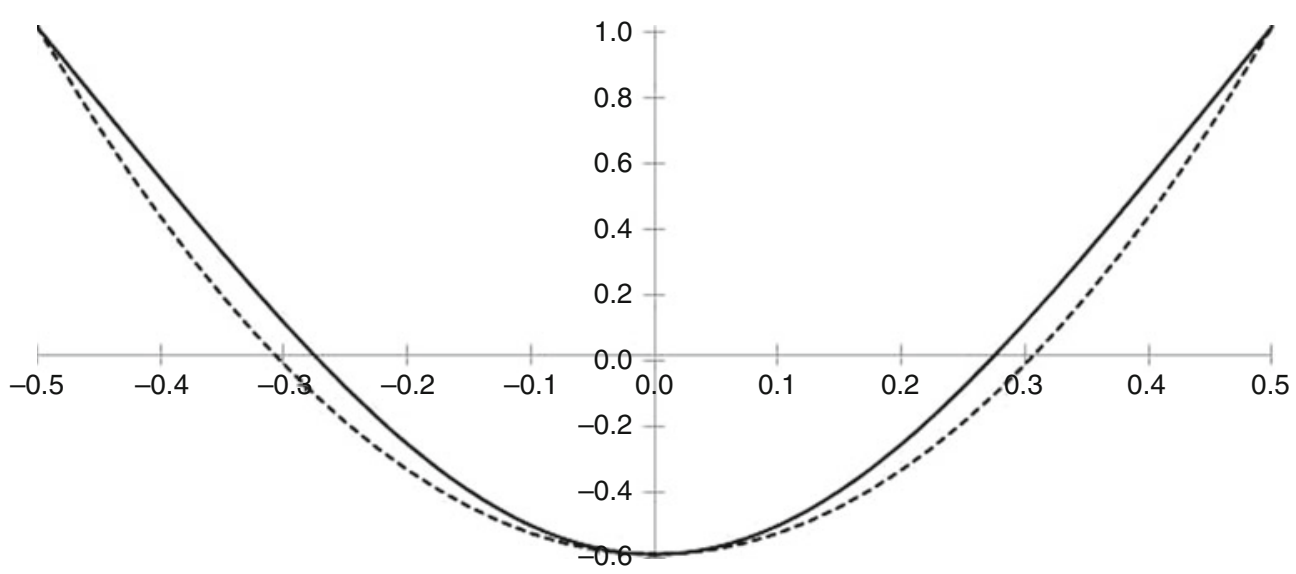

Fig. 5.17 Comparison of the exact solution for a free-free bar vibrating in its fundamental flexural mode (solid line) to the second-order polynomial approximation (dashed line) that is matched to the maximum $y(0)=-0.608$ at $x=0$ and the at the free ends where $y( \pm L / 2)=1$

actual displacements that will be easier to integrate. To exploit the symmetry of the fundamental mode shape, we can place the origin of the coordinate system at the center of the bar.

$$
y_{\text {poly }}(x, t)=A x^{2}+C
$$

At the center of the bar, located at $x=0$, the slope, $\left(\partial y_{\text {poly }} / \partial x\right)_{0}=0$. At the ends of the bar, $x= \pm L / 2$, $\left(\partial^{2} y_{\text {poly }} / \partial x^{2}\right)_{ \pm L / 2}=A / 2 \neq 0$, so the second-order polynomial approximation does not meet the requirement that there be no torques applied to the free end. Since $\left(\partial^{3} y_{\text {poly }} / \partial x^{3}\right)_{ \pm L / 2}=0$, the second-order polynomial approximation does satisfy the boundary condition that requires that there also be no vertical forces.

The displacement of the polynomial approximation can be fit to Eq. (5.64) at $x=0$ by making $C=-0.608$ and fit to the two ends, where $y( \pm L / 2)=1$, by making $A=4(1-C)=6.432$. Figure 5.17 shows both the exact solution of Eq. (5.64) and the second-order polynomial approximation of Eq. (5.66). The kinetic energy of the unloaded bar can be integrated over $-1 / 2 \leq x \leq 1 / 2$, remembering that the vertical motion of the free ends has unit amplitude.

$$
K E_{o}^{F} \cong \frac{\rho L S \omega^{2}}{2} \int_{-L / 2}^{L / 2} y_{\text {poly }}^{2}(x) d x=\left[\frac{A^{2} x^{5}}{5}+\frac{2 A C x^{3}}{3}+C^{2} x\right]_{-L / 2}^{L / 2}=\frac{0.235 M \omega^{2}}{2}
$$

Rayleigh's method will always overestimate the energy, and the $\left|y_{\text {poly }}(x)\right|>|y(x)|$ everywhere, as can be seen in Fig. 5.17 , so I will let $(0.235)^{-1}=4.26 \cong 4$. The kinetic energy of each coil of mass, $m$, at both ends, also with vertical motion of unit amplitude, is $K E_{\text {coils }}=(1 / 2)(2 m) \omega^{2} y_{\text {poly }}^{2}$. From Eq. (5.55), we see that the frequency is inversely related to the square of the length of the bar.

$$
\frac{\delta L}{L}=-\frac{1}{2} \frac{\delta f}{f}=-\frac{1}{2}\left(-\frac{1}{2} \frac{K E_{\text {coils }}}{K E_{o}^{F}}\right)=\frac{1}{4}\left(\frac{4 \Delta M}{M}\right)=\frac{\Delta M}{M} \Rightarrow L_{e f f}^{F}=L\left(1+\frac{\Delta M}{M}\right)
$$

This is in agreement with Rayleigh's result for the flexural mode: "If the load be at the end, its effect is the same as lengthening of the bar in the ratio $M: M+d M$. ." [26]

With the effective lengths removing the systematic error that would be introduced by the mass loading of the transducer coils on the ends of the bar, the moduli can be calculated. The mass ratio is 
$\Delta M / M=(1.5$ grams $/ 55.723$ grams $)=2.7 \times 10^{-2}$. For the longitudinal and flexural modes, $L_{\text {eff }}^{L}=L_{\text {eff }}^{F} \equiv L_{\text {eff }}=34.50 \mathrm{~cm}$. Young's moduli calculated from both methods are determined by Eqs. (5.13) and (5.55).

$$
E_{L}=4 \rho L_{e f f}^{2}\left(\frac{f_{n}^{L}}{n}\right)^{2} \quad \text { and } \quad E_{F}=\left(\frac{32}{\pi}\right)^{2} \frac{\rho L_{e f f}^{4}}{d^{2}}\left(\frac{f_{n}^{F}}{n^{2}}\right)^{2}
$$

Before evaluating $E_{L}$ and $E_{F}$, it will be useful to propagate the relative errors (see Sect. 1.8.4) to obtain an estimate of the accuracy of the results using $\log$ differentiation (see Sect. 1.1.3). It is clear that errors in $\rho, L_{e f f}$, and $f_{n} / n$ are all statistically independent, so the errors are combined in a Pythagorean sum.

$$
\frac{\delta E_{L}}{E_{L}}=\left[\left(\frac{\delta \rho}{\rho}\right)^{2}+\left(2 \frac{\delta L_{e f f}}{L_{e f f}}\right)^{2}+\left(2 \frac{\delta\left(f_{n}^{L} / n\right)}{\left(f_{n}^{L} / n\right)}\right)^{2}\right]^{1 / 2}
$$

Defining the random error as being one standard deviation, $\delta \rho / \rho= \pm 0.32 \%, \delta\left(L_{\text {eff }}\right) / \mid L_{\text {eff }} l= \pm 0.06 \%$, and $\delta\left(f_{n} / n\right) /\left|f_{n} / n\right|= \pm 0.5 \%$. This results in a relative overall uncertainty of $\delta E_{L} / E_{L}= \pm 1.1 \%$. Equation (5.69) can then be evaluated: $E_{L}=5.23 \pm 0.06 \mathrm{GPa}$.

The same procedures can be applied to the calculation of $E_{F}$ and its relative uncertainty.

$$
\frac{\delta E_{F}}{E_{F}}=\left[\left(\frac{\delta \rho}{\rho}\right)^{2}+\left(4 \frac{\delta L_{e f f}}{L_{e f f}}\right)^{2}+\left(-2 \frac{\delta d}{d}\right)^{2}+\left(2 \frac{\delta\left(f_{n}^{F} / n^{2}\right)}{\left(f_{n}^{F} / n^{2}\right)}\right)^{2}\right]^{1 / 2}
$$

Fortunately, the relative uncertainty in effective length is the smallest of the four pieces because it is also the most heavily weighted in Eq. (5.71). The difficulty is the assignment of $\delta d / d$ to account for the ellipticity of the sample's cross-section. I will estimate it to be half the difference in the major and minor diameters over their average, $\delta d / d= \pm\left(d_{\max }-d_{\min }\right) /\left(d_{\max }+d_{\text {min }}\right)= \pm 1.54 \%$. This makes $\delta E_{F} / E_{F}= \pm 3.2 \%$, so from Eq. (5.69), $E_{F}=5.53 \pm 0.18 \mathrm{GPa}$.

Although the lower limit of $E_{F} \geq 5.35 \mathrm{GPa}$ almost overlaps the upper limit of $E_{L} \leq 5.29 \mathrm{GPa}$, their discrepancy is slightly outside our estimate of experimental error. This is probably due to the fact that we represented the elliptical cross-section as a circle with an average diameter $d=\left(d_{\text {max }}+d_{\text {min }}\right) / 2$. The entire discrepancy could possibly be removed if the polarization of the flexural vibrations were rotated by about $30^{\circ}$ toward the $d_{\min }$ orientation. Under the circumstances, if I needed a value of Young's modulus and could not repeat the experiment, I'd use the error-weighted average of the two values and append the larger of the two uncertainties: $E=5.31 \pm 0.18 \mathrm{GPa}$.

To evaluate the shear modulus from the measured torsional mode frequencies, we use Eq. (5.63) to write $L_{e f f}^{T}=35.4 \mathrm{~cm}$.

$$
G=4 \rho\left(L_{e f f}^{T}\right)^{2}\left(\frac{f_{n}^{T}}{n}\right)^{2}
$$

Since this is the same relationship as for the longitudinal standing wave modes, the error propagation is again given by Eq. (5.70), now with $\delta\left(f_{n} / n\right) /\left(f_{n} / n\right)= \pm 0.6 \%$ resulting in $\delta G / G= \pm 2.4 \%$, so from Eq. (5.72), $G=1.77 \pm 0.04 \mathrm{GPa}$. Since $E \cong 3 G$, Poisson's ratio is very close to one-half (see Eq. 4.93). 

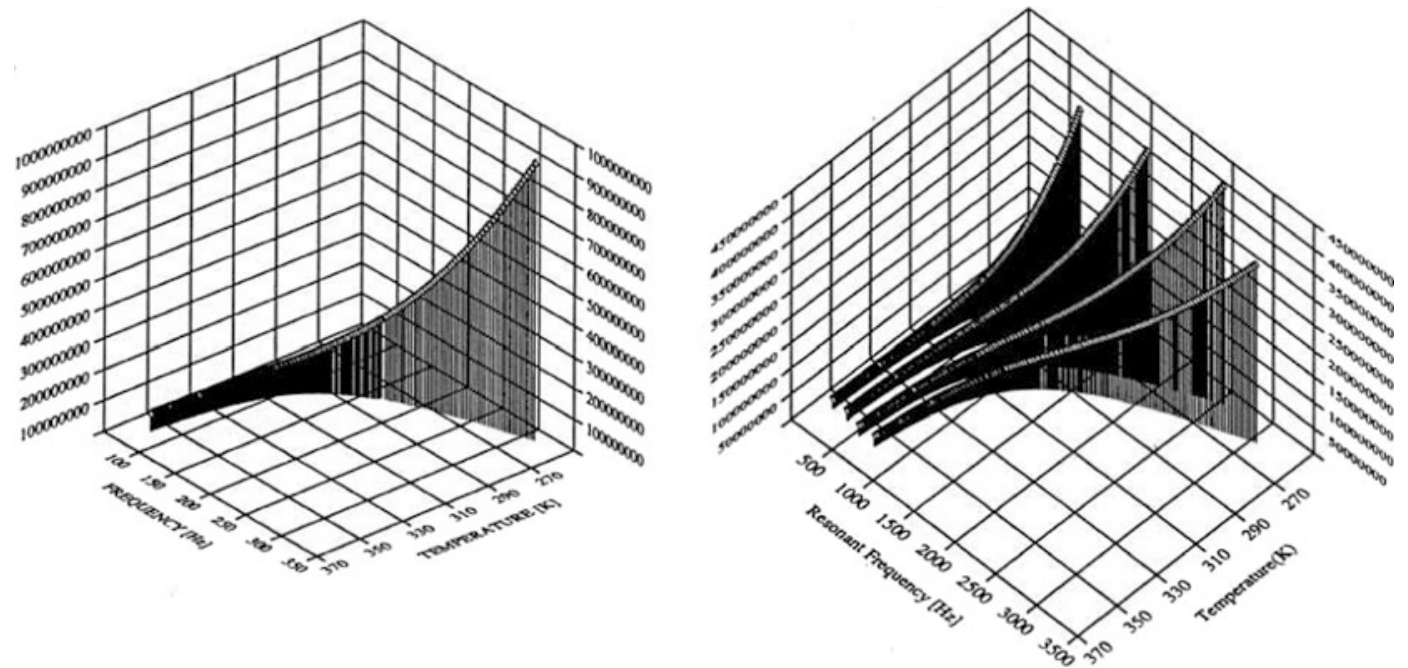

Fig. 5.18 The results of the automated measurement of the lowest-frequency flexural mode (left) and the four lowestfrequency shear modes (right) of an elastomeric bar made of PR 1592 [27], an encapsulant used for hydrophones, over a temperature range from $260 \mathrm{~K}\left(-13{ }^{\circ} \mathrm{C}\right)$ to $360 \mathrm{~K}\left(87^{\circ} \mathrm{C}\right)$. This temperature range includes the material's glass transition temperature. Each point in the temperature-frequency plane represents one measured resonant mode with the height above the point being the elastic modulus corresponding to that temperature and frequency [28]. The viscoelastic behavior is obvious due to the low modulus values at low frequency and high temperature and the high modulus at high frequencies and low temperatures

\subsubsection{Modes of a Viscoelastic Bar}

The previous example demonstrated the simplicity and accuracy of extracting elastic moduli from resonance frequencies of thin bars. Often, it is necessary to determine how those elastic constants vary with some environmental parameter like temperature. Data for a bar sample cast from a viscoelastic elastomer is presented in Fig. 5.18.

The measurements of the resonance frequencies were automated by use of a phase-locked loop frequency tracking system (see Sect. 2.5.3) shown in the block diagram of Fig. 5.19. The sample, with its support structure and the magnets (like Fig. 5.13, but with fine wires providing sample support, replacing the rubber bands) was placed in an environmental control chamber to vary the sample's temperature. The bar is driven by a voltage-controlled oscillator (VCO) that produces a sinusoidal voltage that is amplified and applied to the drive coil. The signal from the receive coil is then passed through a pre-amplifier that has a high-pass filter to remove low-frequency noise and vibration. Of course, all of the electronics instruments are outside the environmental chamber.

The frequency of the VCO is controlled to keep the bar vibrating at one of its free-free normal mode frequencies by controlling the relative phase between the drive voltage and the received signal voltage. The force on the driven coil, $F(t)=(B \ell) I(t)$, is in phase with the current or voltage, since the coil's electrical impedance is primarily resistive. The output of an electrodynamic transducer is governed by Faraday's law, as expressed in Eq. (5.57), so the receive coil's velocity (linear or angular) and the force should be in-phase at resonance; hence, those two voltages should be in-phase. ${ }^{19}$

\footnotetext{
${ }^{19}$ Unlike the example in Sect. 2.5.3, which controlled a single degree-of-freedom simple harmonic oscillator, the bar has standing wave modes, so the phase relation between force and velocity will alternate by $180^{\circ}$ between adjacent modes.
} 


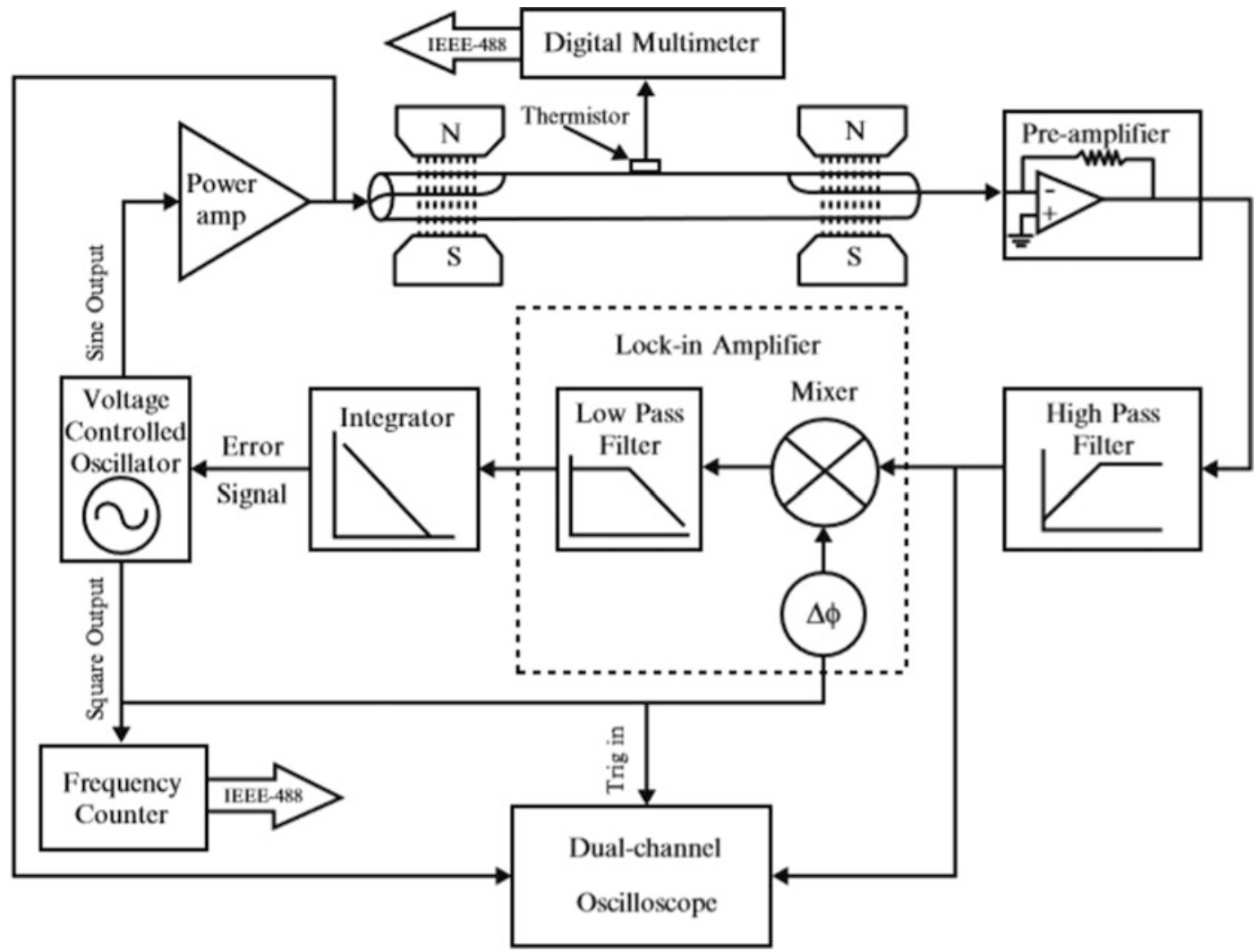

Fig. 5.19 Block diagram of the instrumentation and electronics [19] used to automatically track the resonance frequencies and amplitudes for a vibrating bar to produce results like those shown in Fig. 5.18

A two-phase lock-in amplifier [29] was used in this application. The algorithm could be based on a simple pair of analog multiplier chips, such as the analog Devices AD633, or it could be accomplished entirely in software. One phase will generate the error signal that is integrated and fed back to the input of the VCO. Integration of the error signal allows the integrator to "store" the required voltage to tune the VCO to the resonance frequency, since the error signal voltage will be zero when the frequencytracking system is locked onto the resonance frequency.

The other (quadrature) output signal from the lock-in will be proportional to the amplitude, since the relative phases of the two lock-in outputs will differ by $90^{\circ}$. With the feedback network forcing the output of the frequency control channel to zero, the entire signal will appear at the quadrature output from the other lock-in channel. Because of the low-pass filters on the outputs of the mixers (i.e., multipliers), the effective equivalent noise bandwidth $(\Delta f)_{E Q N B}$ (see Sect. 2.5.2), even for a singlestage low-pass (RC) filter, with a roll-off of $-6 \mathrm{~dB}$ /octave, will have $(\Delta f)_{E Q N B}=(\pi / 2)(\Delta f)_{-3 d B}$ (see Fig. 2.8). As more stages are added, the coefficient decreases from $\pi / 2$ to 1 . For a two-stage filter (i.e., $-12 \mathrm{~dB}$ /octave), the $(\Delta f)_{E Q N B}=1.22(\Delta f)_{-3 d B}$. For measurements that are slow, due to long thermal equilibration times, the time constant, $\tau_{-3 d B}$, of the low-pass filter can be set to $10 \mathrm{~s}$, so $(\Delta f)_{-3 \mathrm{~dB}}$ is $\left(2 \pi \tau_{\text {. }}\right.$ $3 d B)^{-1}=16 \mathrm{mHz}$ making $(\Delta f)_{E Q N B}=25 \mathrm{mHz}$, even for a single-stage low-pass filter.

Inspection of the data in Fig. 5.18 shows the "cleanliness" that can be achieved with this resonance frequency tracking strategy. There are some challenges in setting up the system so that the gains of the amplifiers and their associated phase shifts (an inevitable consequence of the Kramers-Kronig relations), as well as the phase shifts caused by the low-pass filters and integrator, do not lead to positive feedback and oscillatory behavior in the control system. For a laboratory system, it is easy to 
adjust the amplifier gains and the filter time constants since these are just knobs or rotary switches on the front panel of the lock-in amplifier. For a production system, it is useful to do a careful gain-phase analysis for all the components [30].

If we hold the current that is applied to the driven coil constant, so that its amplitude is independent of frequency, then the driving force (or torque) will remain constant. In that case, the magnitude of the response measured by the receiver transducer will be proportional to the quality factor, $Q$, at resonance and inversely proportional to the internal dissipation of the material. Such amplitude data was collected and recorded simultaneously with the modal frequencies of Fig. 5.18.

The data shown in Fig. 5.18 combines both the modulus and the damping from the resonance frequency and amplitude measurements made on the PR 1592 [27] elastomeric bar sample. The frequency data for a torsional mode was converted to the shear modulus, as was done for the epoxyglass composite sample using Eq. (5.72). Similarly, the amplitude data was converted to loss tangent. Since the data was acquired over a range of temperatures that included the glass transition temperature, $T_{\text {glass }}$, the effective range of frequencies for this measurement is over ten orders of magnitude, although the actual frequencies only varied by about a factor of four, as seen in Fig. 5.18.

The horizontal axis in Fig. 5.20 is labeled reduced frequency, not frequency. As seen in our analysis of viscoelastic materials in Sect. 4.4, the influence of frequency on the response of a viscoelastic material only shows up in the equations as a product with the relaxation time, $\tau_{R}$. The behavior depends upon $\omega \tau_{R}$ rather than on $\omega$ directly. The reason that the range of reduced frequencies is so enormous in Fig. 5.20 is that viscoelastic relaxation time, $\tau_{R}$, is a very strong function of temperature.

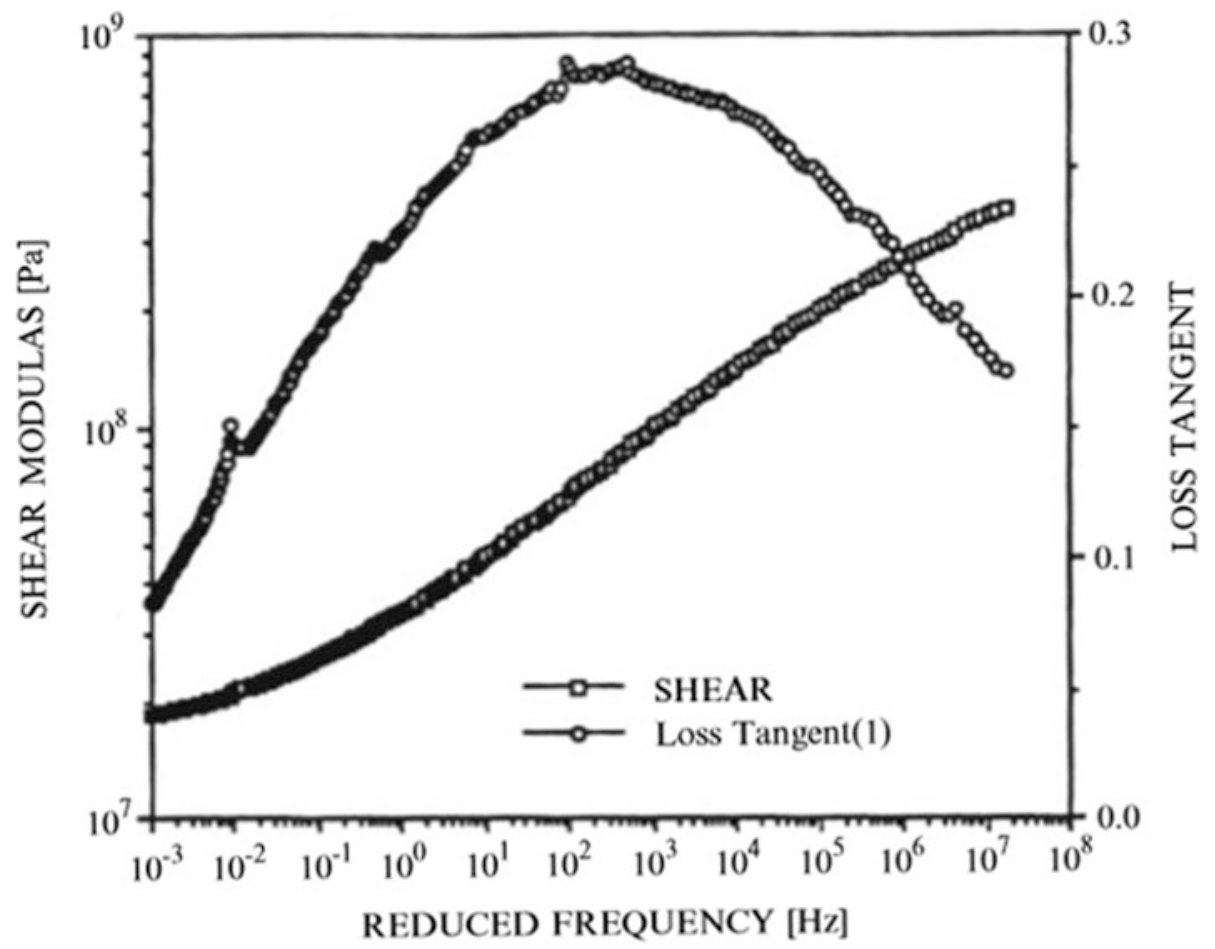

Fig. 5.20 Master curve for the magnitude of the complex shear modulus and the associated loss tangent plotted vs. the reduced frequency. This plot combines both frequency and temperature variations using the Williams-Landel-Ferry transformation of Eq. (5.73) to produce over ten decades span in reduced frequency. This plot clearly illustrates the single relaxation time behavior introduced in Sect. 4.4. The reference temperature for this graph is $10{ }^{\circ} \mathrm{C}$. At that temperature, the actual frequencies and reduced frequencies are identical 
As shown in Fig. 4.28, most of the changes in both modulus and loss occur in the regions that are close to the glass transition temperature, being the temperature where $\omega \tau_{R} \cong 1$. This dependence on both frequency and temperature can be converted to a single parameter that is proportional to $\omega \tau_{R}$ rather than to either $\omega$ or $\tau_{R}(T)$, which is what was done to produce the reduced frequency that is plotted on the horizontal axis of Fig. 5.20. Such a curve is called the master curve, since it can provide the complex modulus at any frequency, if the temperature is known, or the effect a change in temperature will make on the complex modulus at a fixed frequency.

It is obvious from inspection of the plots of moduli in Fig. 5.18 that the measured moduli are much smaller at low frequencies and high temperatures, while the moduli are greatest at high frequencies and low temperatures. There are several algorithms that can be used to convert a frequency measurement made at a temperature, $T$, to reduced frequency, so it can be plotted with other frequency measurements made at other temperatures. The Williams-Landel-Ferry (WLF) equation [31] was used to produce the graph in Fig. 5.20 by creating a frequency shift factor, $\alpha_{T}$, so that data acquired at a frequency, $f$, and at a temperature, $T$, are plotted on the reduced frequency axis at a reduced frequency, $f^{\prime}=\alpha_{T} f$.

$$
\ln \alpha_{T}=\frac{-C_{1}\left(T-T_{\text {glass }}\right)}{C_{2}+\left(T-T_{\text {glass }}\right)}
$$

The constants $C_{1}, C_{2}$, and $T_{\text {glass }}$ are different for different polymers, but, as the form of Eq. (5.73) suggests, the dependence of $\tau_{R}(T)$ is nearly an exponential function of the difference between the measured temperature, $T$, and $T_{\text {glass }}$.

\subsubsection{Resonant Ultrasound Spectroscopy*}

“So, apparently due to a mathematical fortuity that may have occurred during a lapse in Murphy's vigilance, the
displacement vectors, $u_{i}$, which are solutions to the elastic wave equation with free boundary conditions on S, are
just those points in function space at which L is stationary.” W. M. Visscher [32]

The measurement of the elastic moduli of a long thin bar, as described in the previous sections, was simplified because the longitudinal, torsional, and flexural modes of such a bar were distinct. Furthermore, the modes could be excited and detected selectively by the orientation of the electrodynamic transducers with respect to the magnetic field. If a sample is not available in the shape of a long thin bar, it is still possible to determine the elastic moduli from measured resonance frequencies, but the extraction of the moduli from the resonance spectrum is much more difficult and computationally intensive.

In the early 1990s, a technique was developed that allows the normal mode frequencies of an object of arbitrary shape, and possibly anisotropic elasticity, to be accurately approximated if the object is freely suspended (hence, the quote that started this section regarding the "lapse in Murphy's vigilance"). More importantly, measurement of the resonance frequencies of such normal modes of vibration can be inverted to extract the elastic moduli of the sample. This technique has become known as resonant ultrasound spectroscopy (RUS) [33].

Figure 5.21 shows an apparatus that can excite and detect the resonances of a very small solid rectangular parallelepiped sample (in this case). The normal modes of oscillation for such a rectangular sample are represented in Fig. 5.22. As we have done before, the "trick" is to use polynomial functions that are products of powers of the three Cartesian coordinates, where $\lambda=\ell, m$, and $n$ is a set of three nonnegative integers.

$$
\Phi_{\ell, m, n}=x^{\ell} y^{m} z^{n} \equiv \Phi_{\lambda}
$$

These functions are then used to express the displacements, $u_{i}$, along the three Cartesian directions. 

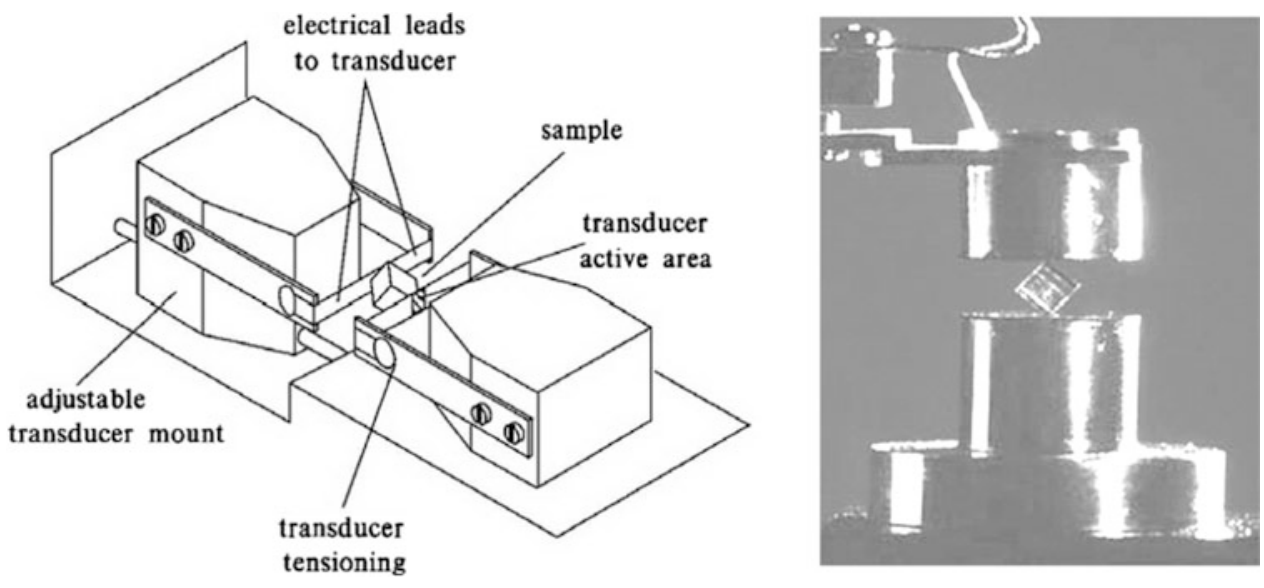

Fig. 5.21 (Left) Schematic diagram of an apparatus that is capable of holding a very small and fragile solid sample between two piezoelectric polymer film (PVDF) transducers, $0.50 \mathrm{~mm}$ wide, that only touch the sample at the corners and can excite and detect the sample's normal modes of vibration [33, 34]. (Right) Photograph of a room temperature RUS apparatus that excites the sample using ordinary piezoelectric transducers

$$
u_{i}=\sum_{\lambda \in \Omega} a_{i \lambda} \Phi_{\lambda}
$$

The number of such functions must be sufficient for the number of modes to be determined, so that the energy can be minimized through the proper choice of the $a_{i \lambda}$ using standard matrix methods. This polynomial approach will work regardless of the shape of the sample, as long as the motions of its boundaries are not constrained. The paper by Visscher et al. provides a rather entertaining description of the variety of such shapes: "including spheres, hemispheres, spheroids, ellipsoids, cylinders, eggs, shells, bells, sandwiches ${ }^{20}$, parallelepipeds, cones, pyramids, prisms, tetrahedra, octahedra, and potatoes." [32]

The execution of these matrix operations was originally rather time-consuming or required a supercomputer. The examples shown in the paper by Visscher et al., in 1991, required a Cray-1 computer. Only 20 years later, the necessary calculations can be executed on a laptop computer. The results can be inverted to provide values of the elastic constants and their uncertainties, based on the measured resonance frequencies.

RUS has been extraordinarily successful in measuring the elastic properties of very small samples. Spoor and Maynard were able to measure the elastic moduli of a sample of quasicrystalline AlCuLi that had a mass of only 70 micrograms [34]. This is necessary because it is frequently difficult to grow large crystalline samples of exotic materials like quasicrystals or high-temperature superconductors. The simplicity and compactness of the apparatus also make RUS well-suited to measurements of the temperature dependences of elastic moduli and/or measurement on samples that are potentially dangerous.

My favorite examples are the studies on the solid phases of plutonium done by Albert Migliori and his colleagues at Los Alamos National Laboratory. ${ }^{21}$ Plutonium exhibits four different solid phases over a relatively small range of temperatures. Shown in Fig. 5.23 are measurements of the bulk and

\footnotetext{
${ }^{20}$ Visscher was incorrect about "sandwiches." Polynomials cannot fit a sharp interface, so the results are too inaccurate for RUS. For a sharp interface, finite elements are required.

${ }^{21}$ Large samples of plutonium self-heat by radioactive decay (or worse!).
} 


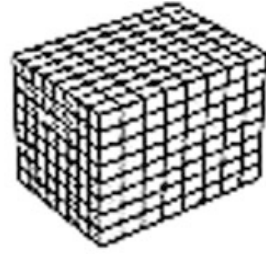

ar rese

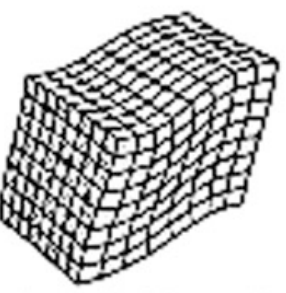

CX-1 (5.234 NG2)

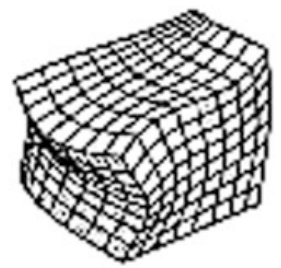

EZ-1 (6.270 $501 \times)$

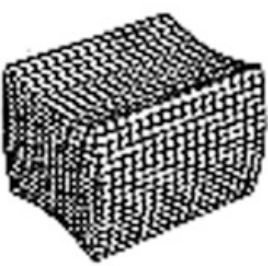

05.3 (6.898 मH/x)

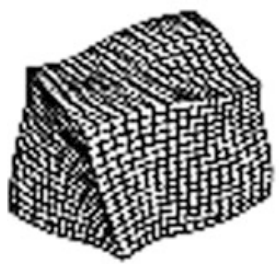

EV-3 (7.652 $\mathrm{xHz}$ )

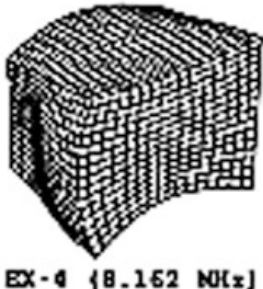

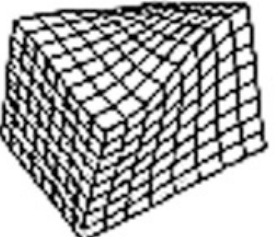

EV. 1 (3.296 valx)

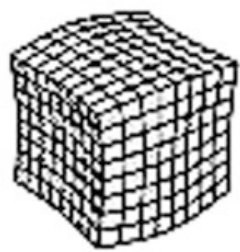

CD-1 (5.389 y.He)

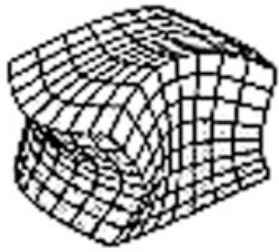

$\infty 0.2(6.332 \mathrm{kHz})$

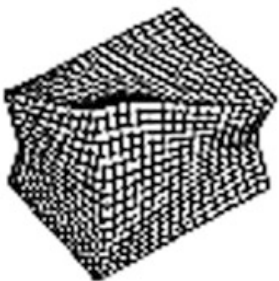

$0 \times .2$ (6.928 $\mathrm{hKz})$

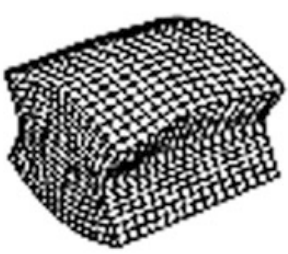

OD.5 \{7.751 NRx]

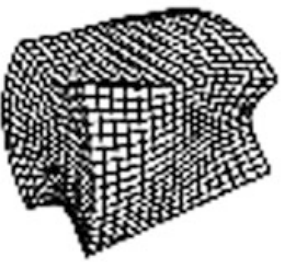

22.4 $\{8,359 \mathrm{MKr}\}$

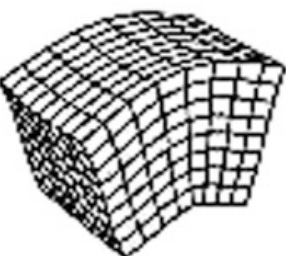

GT-1 (2.213 182)

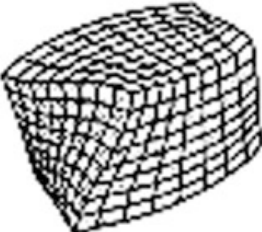

$0 z \cdot 1$ (S. 8ร7 $\% \mathrm{~Hz})$

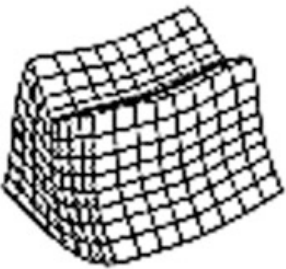

EX-2 $\{6.381 \mathrm{HHx}\}$

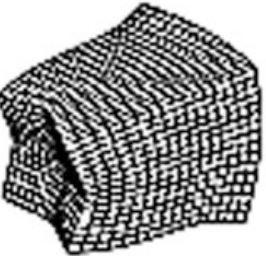

Z2-2 17.120 NOF

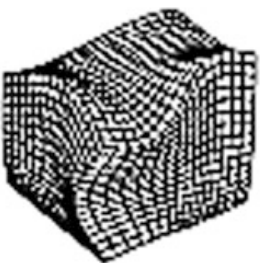

82-3 [7,851 $\mathrm{kER}$ ]

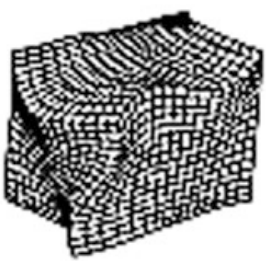

or-3 (8.389 몬)

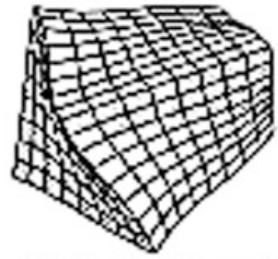

FV.2 (4.805 बa्a)

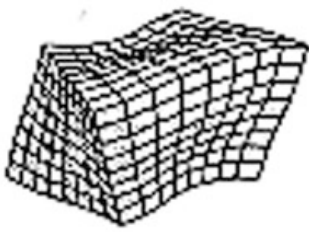

$0 z-2(6.196 \mathrm{H} / \mathrm{x})$

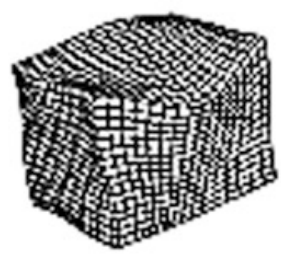

$0 z \cdot 3\langle 6.450 \mathrm{bHz}$

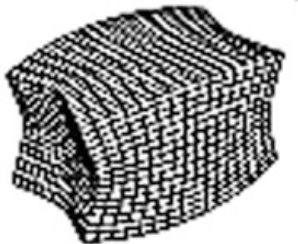

OD.4 I?.225 $\mathrm{kBz}$

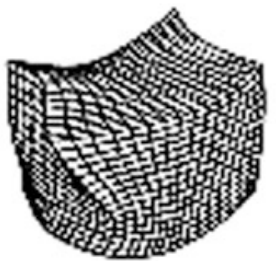

EX-3 [7,896 kog ।

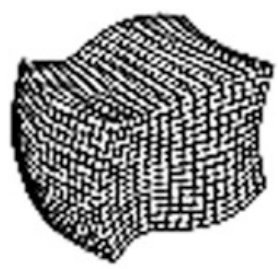

22.5 (8.494 Yब2)

Fig. 5.22 Normal mode vibrational patterns for a rectangular parallelepiped showing that longitudinal, flexural, and torsional motions are combined in several of the modes [36]. By fitting the polynomial mode shapes, it is possible to isolate the individual elastic moduli, even for materials with anisotropic elastic properties, as discussed in Sect. 4.6, to high accuracy 

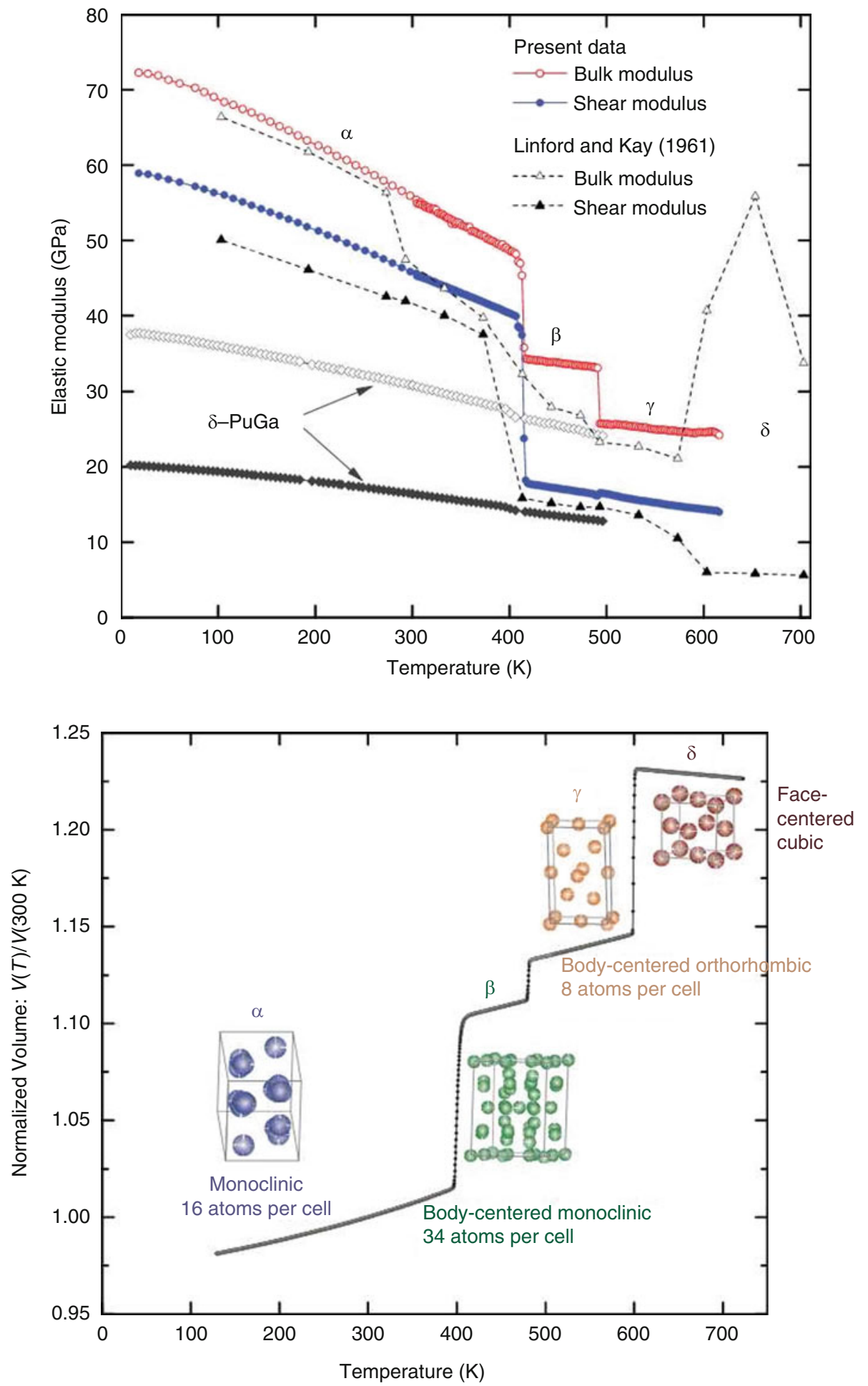

Fig. 5.23 (Above) Shear and bulk moduli of plutonium clearly showing the transitions between the various solid phases. (Below) Plutonium's density transitions for four solid phases including diagrams of the atomic arrangements for those phases [35] 
shear moduli of pure Pu from $18 \mathrm{~K}$ to $616 \mathrm{~K}$ using RUS [35]. As with other resonant determinations of elastic moduli, acoustic measurements provide extraordinary data density and unmatched precision.

\subsection{Vibrations of a Stiff String*}

All of the calculations made in Chap. 3, which developed our understanding of the dynamics of wave motion on strings, assumed that the string was "limp," meaning that only tension provided the restoring force-the string itself had no flexural rigidity $\left(E=0\right.$, so $\left.E S \kappa^{2}=0\right)$. Anyone who has replaced the strings on a guitar and poked the end of a finger with the stiff string knows differently; guitar strings have sufficient rigidity to penetrate the skin (painfully). Now that we have analyzed the flexure of bars, where the only restoring force is the rigidity of the bar, we are in a position to calculate the effects that rigidity adds to the behavior of strings that are also under tension.

We have already done all of the necessary work. The vertical acceleration of a differential element of a stiff string will just be the sum of the vertical forces as calculated previously in Eq. (3.3) for the tension and in Eq. (5.36) for the flexural rigidity.

$$
\rho S \frac{\partial^{2} y}{\partial t^{2}}=\mathrm{T} \frac{\partial^{2} y}{\partial x^{2}}-E S \kappa^{2} \frac{\partial^{4} y}{\partial x^{4}} \Rightarrow \frac{\partial^{2} y}{\partial t^{2}}-c_{s t}^{2} \frac{\partial^{2} y}{\partial x^{2}}+c_{B}^{2} \kappa^{2} \frac{\partial^{4} y}{\partial x^{4}}=0
$$

Recall that the linear mass density of a string is $\rho_{L}=\rho S$. The speed of transverse waves on a limp string will be designated $c_{s t}=\left(\mathrm{T} / \rho_{L}\right)^{1 / 2}$, to distinguish it from the speed of longitudinal waves in thin bars, $c_{B}=(E / \rho)^{1 / 2}$.

As in Sect. 5.3.1, the assumption of a rightward-going traveling wave solution of the form $y(x, t)=\mathfrak{R} e\left[\widehat{\mathbf{C}} e^{j}(\omega t-k x)\right]$ will lead to an equation that yields the dispersion relation that combines tension and stiffness.

$$
c_{B}^{2} \kappa^{2} k^{4}-c_{s t}^{2} k^{2}-\omega^{2}=0
$$

The solution of this characteristic equation is just that for a quadratic equation in $k^{2}$.

$$
k^{2}=\frac{c_{s t}^{2} \pm \sqrt{c_{s t}^{4}+4 c_{B}^{2} \kappa^{2} \omega^{2}}}{2 c_{B}^{2} \kappa^{2}}=\frac{c_{s t}^{2}}{2 c_{B}^{2} \kappa^{2}}\left[1 \pm \sqrt{1+\frac{4 \omega^{2} c_{B}^{2} \kappa^{2}}{c_{s t}^{4}}}\right]
$$

We know that the phase speed, $c_{p h}$, for a flexural wave depends upon the sharpness of the bend. As derived in Eq. (5.38), $c_{p h}=\left(2 \pi c_{B} \kappa\right) / \lambda$ and $c_{s t}$ is independent of wavelength. We expect that in the limit of very low frequencies, hence, very long wavelengths, the tension should be the dominant restoring force. A binomial expansion can be used for $\left(4 \omega^{2} c_{B}{ }^{2} \kappa^{2} / c_{s t}^{4}\right) \ll 1$ to illustrate this limit.

$$
\lim _{\omega^{2} \rightarrow 0}\left[k^{2}\right]=\frac{c_{s t}^{2}}{2 c_{B}^{2} \kappa^{2}}\left[1 \pm\left(1+\frac{2 \omega^{2} c_{B}^{2} \kappa^{2}}{c_{s t}^{4}}\right)\right]=\frac{\omega^{2}}{c_{s t}^{2}}
$$

As expected, for very long wavelengths, the phase speed, $c_{p h}=\omega / k$, is constant and equal to the speed of transverse waves on a limp string, $c_{s t}$.

In the opposite limit of very high frequencies and short wavelengths, we expect the phase speed, $c_{p h}$, that is characteristic of flexure waves on rigid bars that are not under tension. 


$$
\lim _{\omega^{2} \rightarrow \infty}\left[k^{2}\right]=\frac{\omega}{c_{B} \kappa} \quad \Rightarrow \quad c_{p h} \equiv \frac{\omega}{k}=c_{B} \kappa k=\sqrt{c_{B} \kappa \omega}
$$

This result is identical to Eqs. (5.38) and (5.39).

The solutions to Eq. (5.76) can be found by again using separation of variables, as we did in Sect. 5.3.2, with $y(x, t)=Y(x) T(t)=Y e^{k x} T e^{j \omega t}$. This substitution converts Eq. (5.76) from a partial differential equation to an ordinary differential equation.

$$
\frac{d^{4} Y}{d x^{4}}-\frac{c_{s t}^{2}}{c_{B}^{2} \kappa^{2}} \frac{d^{2} Y}{d x^{2}}-\frac{\omega^{2}}{c_{B}^{2} \kappa^{2}}=0 \Rightarrow k^{4}-\left(\frac{c_{s t}^{2}}{c_{B}^{2} \kappa^{2}}\right) k^{2}-\frac{\omega^{2}}{c_{B}^{2} \kappa^{2}}=0
$$

The definition of two new constants, $2 \beta^{2}$ and $\gamma^{4}$, simplifies the application of the quadratic formula to Eq. (5.81).

$$
k^{4}-2 \beta^{2} k^{2}-\gamma^{4}=0, \quad \text { where } \quad 2 \beta^{2} \equiv \frac{c_{s t}^{2}}{c_{B}^{2} \kappa^{2}} \text { and } \gamma^{4} \equiv \frac{\omega^{2}}{c_{B}^{2} \kappa^{2}}
$$

As before, taking the fourth root of $k^{4}$ will generate four values of wavenumber, although in this case, we need to use the quadratic formula to find $k^{2}$.

$$
\begin{aligned}
k_{ \pm}^{2}= & \frac{2 \beta^{2} \pm \sqrt{4 \beta^{4}+4 \gamma^{4}}}{2}=\beta^{2} \pm \beta^{2} \sqrt{1+\frac{\gamma^{4}}{\beta^{4}}} \\
& \text { so } \\
k_{+}^{2}= & \beta^{2}\left[1+\sqrt{1+\frac{\gamma^{4}}{\beta^{4}}}\right] \text { and } k_{-}^{2}=\beta^{2}\left[1-\sqrt{1+\frac{\gamma^{4}}{\beta^{4}}}\right]
\end{aligned}
$$

Since $(\gamma / \beta)^{4}=4 c_{p h}^{4} / c_{s t}^{4}>0$, the square roots of $k_{+}{ }^{2}$ will be real numbers, and the square roots of $k_{-}{ }^{2}$ will be purely imaginary numbers. Substitution of these results back into $\mathrm{Y}(x)$ produces four exponentials that are similar to those in Eq. (5.41), but with the hyperbolic trigonometric functions having $k_{+} x$ in their argument and the circular trigonometric functions using $k_{-} x$.

$$
\begin{aligned}
& \mathrm{Y}(x)=C_{1} e^{k_{+} x}+C_{2} e^{-k_{+} x}+C_{3} e^{j k_{-} x}+C_{4} e^{-j k_{-} x} \text { or } \\
& \mathrm{Y}(x)=A \cosh \left(k_{+} x\right)+B \sinh \left(k_{+} x\right)+C \cos \left(k_{-} x\right)+D \sin \left(k_{-} x\right)
\end{aligned}
$$

At this point, we could proceed by fitting boundary conditions, generating transcendental equations that quantize the allowable values of $k_{n}$ for normal mode vibrations, and calculating the resonance frequencies, $\omega_{n}$, of those modes. Having done this once before for the flexural modes of bars (and having found it to be tedious!), we will not go down that route here again. Those readers who are nostalgic for such algebraic gymnastics are referred to Morse [37], although his final result is incorrect. ${ }^{22}$

Since our goal is to determine the change in the modal frequencies and resulting anharmonicity of the overtones for a fixed-fixed string due to its flexural rigidity, ${ }^{23}$ we can assume that tension is the

\footnotetext{
${ }^{22}$ Morse's solution for the frequency in his result for his $\nu_{n}$, equal to our $f_{n}$, includes a mode-independent constant term. He is not able to produce the $n^{2}$ dependence without adding another term to his Taylor series expansion, and his result is obviously incompatible with Young's observations [38].

${ }^{23}$ Piano technicians compensate for this anharmonicity. Anharmonicity is present in different amounts in all of the ranges of the instrument but is especially prevalent in the bass and high treble registers. The result is that octaves are tuned slightly wider than the harmonic 2:1 ratio. The exact amount that octaves are "stretched" by a piano tuner, by tuning the octave to a match half the frequency of the second overtone instead of the first, varies from piano to piano and even from register to register within a single piano-depending on the exact anharmonicity of the strings involved. With small pianos, the anharmonicity is so significant that they are stretched by matching the triple-octave.
} 
dominant restoring force (since tension is what is adjusted to tune the stiff string's pitch). Additionally, the transverse displacement function, $y(x, t)$, of the string is not significantly affected by the string's flexural rigidity.

By now, the previous statements should suggest to the serious reader the application of Rayleigh's method, using the mode shapes, $y_{n}(x, t)$, for a fixed-fixed string in Eq. (3.21), repeated here, as the trial functions.

$$
y_{n}(x, t)=\mathfrak{R} e\left[C_{n} e^{j \omega_{n} t} \sin \left(n \frac{\pi x}{L}\right)\right] ; \quad n=1,2,3, \ldots
$$

We have already demonstrated that Rayleigh's method provides the exact solution for the modal frequencies, $\omega_{n}$, of limp fixed-fixed string in Eq. (3.32) by taking the square root of the ratio of the stability coefficient to the inertia coefficient. Since the addition of flexural rigidity does not change the linear mass density, $\rho_{L}$, of the string, the addition of flexural rigidity to the tension as a restoring force means that the relative change in frequency, $\delta f_{n} / f_{n}$, will be determined by the relative change in the stability coefficient (the potential energy) alone.

$$
\frac{\delta f_{n}}{f_{n}}=\frac{1}{2} \frac{\delta(P E)_{n}}{\left(P E^{T}\right)_{n}}=\frac{1}{2} \frac{\left(P E^{F}\right)_{n}}{\left(P E^{T}\right)_{n}}
$$

The expression for the potential energy due to flexure of a differential element of length, $\mathrm{d} x$, given by $\mathrm{d}\left(P E^{F}\right)$, that is bent by an angle, $\phi$, is provided in Eq. (4.32). This expression can be integrated over the length, $L$, of the string using the fixed-fixed mode shapes of Eq. (5.85).

$$
\begin{gathered}
\left(P E^{F}\right)_{n}=\frac{E S \kappa^{2}}{2} \int_{0}^{L}\left(\frac{\partial^{2} y_{n}}{\partial x^{2}}\right)^{2} d x=\frac{E S \kappa^{2}}{2}\left(\frac{n \pi}{L}\right)^{4} C_{n}^{2} \int_{0}^{L} \sin ^{2}\left(\frac{n \pi x}{L}\right) d x \\
\Rightarrow \quad\left(P E^{F}\right)_{n}=n^{4} \pi^{4} C_{n}^{2} \frac{E S \kappa^{2}}{4 L^{3}}
\end{gathered}
$$

The maximum potential energy, $\left(P E^{T}\right)_{n}$, in mode $n$ for the limp string was provided in Eq. (3.31) and reproduced here.

$$
(P E)_{n}=\frac{\pi^{2} \mathrm{~T} L}{\lambda_{n}^{2}} C_{n}^{2}=\frac{n^{2} \pi^{2} \mathrm{~T}}{4 L} C_{n}^{2}
$$

The relative frequency shift, $\delta f_{n} / f_{n}$, for any mode number, $n$, is given by substitution of Eqs. (5.87) and (5.88) into Eq. (5.86).

$$
\frac{\delta f_{n}}{f_{n}}=\frac{1}{2} \frac{\left(P E^{F}\right)_{n}}{\left(P E^{T}\right)_{n}}=\frac{(\pi n)^{2}}{2} \frac{E S}{\mathrm{~T}}\left(\frac{\kappa}{L}\right)^{2}=\frac{(\pi n)^{2}}{2 L^{2}} \frac{c_{B}^{2} \kappa^{2}}{c_{s t}^{2}}
$$

This solution reproduces the result of Young who claimed that the measurements were "entirely compatible with the relationship given" [38]. The effects of the string's stiffness grow with the square of the mode number, $n$. The dimensionless ratios, $E S / \mathrm{T}$ and $(\kappa / L)^{2}$, scale the importance of the flexural rigidity and the tension as restoring forces.

We can now do an example to estimate the anharmonicity for a piano string based on Eq. (5.89). The speaking length, ${ }^{24} L$, of the piano string, corresponding to "middle-C," with a frequency of

\footnotetext{
${ }^{24}$ The "speaking length" of a piano string is the distance between the bridge, located on the sound board near the hitching pin, and the capo d'astro, near the tuning pin. It is the speaking length that determines the distance between the fixedfixed boundaries.
} 
$f=261.6 \mathrm{~Hz}$, is $63 \mathrm{~cm}$. Strings are typically made from high-carbon steel (e.g., ASTM A228) with a Young's modulus of $E=210 \mathrm{GPa}$ and mass density of $\rho=7850 \mathrm{~kg} / \mathrm{m}^{3}$. The string is tensioned to about $\mathrm{T}=780 \mathrm{~N}$. Assuming the tension dominates the flexural rigidity, the speed of transverse vibrations is $c_{s t}=f \lambda=2 f L=332 \mathrm{~m} / \mathrm{s}$. The linear mass density of the string can be related to the tension and $c_{s t}: \rho_{L}=\mathrm{T} / c_{s t}{ }^{2}=7.07 \times 10^{-3} \mathrm{~kg} / \mathrm{m}$. Using the mass density of steel, $\rho_{\text {steel }}$, the diameter of the wire is also determined, $d=\left(4 \rho_{L} / \pi \rho_{\text {steel }}\right)^{1 / 2}=1.07 \mathrm{~mm}$; hence, $\kappa^{2}=a^{2} / 4=d^{2} / 16=7.17 \times 10^{-8} \mathrm{~m}^{2}$.

Young's dimensionless anharmonicity coefficient [38], $b$, can be expressed in terms of $d$ using Eq. (5.89).

$$
b=\frac{\pi^{3}}{128} \frac{E}{\mathrm{~T}}\left(\frac{d^{2}}{L}\right)^{2}
$$

Substituting the nominal values calculated for the middle-C string of a piano, $b=2.15 \times 10^{-4}$, with $\delta f_{n} / f_{1}=n^{2} b$. The first overtone, $n=2$, at the octave above middle-C is therefore sharp by $861 \mathrm{ppm}$, or about 1.5 cents. ${ }^{25}$ In Table 3.2, we saw that the $n=5$ and $n=7$ harmonics did not correspond exactly to a just-temperament interval, with $n=5$ falling between $E^{\#}$ and $F^{b}$ and $n=7$ falling between $A^{\#}$ and $B^{b}$ for a scale based on middle-C. Those harmonics will be up-shifted by $5.375 \times 10^{-3}$ (9.3 cents) and $1.011 \%$ ( 17.5 cents), respectively.

This example set an effective lower limit on the anharmonicity of stiff piano strings, since the anharmonicity is greatest in the upper and lower registers of the piano, farthest from middle-C.

\subsection{Harmonic Analysis}

In this chapter, we used our understanding of elasticity from the previous chapter to describe the propagation of waves in solids and used our understanding of the modal frequencies of those standing waves in solids to evaluate the elastic moduli introduced in the previous chapter-a fair exchange. More importantly, we saw in this chapter the power of harmonic analysis (i.e., the assumption of time harmonic solutions) as augmented by a more formalized version of the approach known as separation of variables. That perspective not only was useful for solutions to the wave equations but also to the solution of higher-order partial differential equations. Imposition of boundary conditions again resulted in the quantization of normal mode frequencies in the flexural cases, though requiring four boundary conditions, rather than two required for systems that obeyed the wave equation.

We are now fully equipped to take our techniques for analyzing zero-dimensional harmonic oscillators and one-dimensional systems (strings and bars) and extend them to two-dimensional membranes and plates.

Talk Like an Acoustician

$\begin{array}{ll}\text { Flexural rigidity } & \text { Dynamic range } \\ \text { Polar moment of inertia } & \text { Electromagnetic cross talk } \\ \text { Torsional rigidity } & \text { Quadrature output } \\ \text { Phase speed } & \text { Reduced frequency } \\ \text { Dispersion } & \text { Master curve } \\ \text { Pure tone } & \text { Resonant ultrasound spectroscopy } \\ \text { Separation of variables } & \text { Speaking length (for piano strings) }\end{array}$

\footnotetext{
${ }^{25}$ Recall from Sect. 3.3.3 that one cent is one one-hundredth of an equal-temperament semitone (in the logarithmic sense), or a frequency ratio of $2^{1 / 1200}=1.000578$.
} 


\section{Exercises}

1. Longitudinal vibration of a fixed-mass loaded bar. The longitudinal vibrational normal modes of a thin bar with fixed-fixed and free-free boundary conditions were calculated in Sect. 5.1. In this exercise, you will calculate the longitudinal modes of a bar of length, $L$, and cross-sectional area, $S_{x}$, that is fixed at $x=0$ and has a mass, $M$, attached at $x=L$.

(a) Normal mode frequencies. If the added mass, $M$, has twice the mass of the bar, $m_{B}=(M / 2)$, calculate the first three normal mode frequencies in terms of $c_{B}$ and $L$.

(b) Hooke's law limit. Approximate the frequency of the gravest mode (i.e., the $f_{o}$ mode corresponding nearly to a mass-spring oscillator) by assuming that the mass, $M$, is restored by a stiffness, $\mathrm{K}=E S_{x} / L$. Also report these results in terms of $c_{B}$ and $L$.

(c) Effective mass approximation. Use a Taylor series expansion [similar to the technique that produced Eq. (3.71)] to determine $f_{o}$ by adding the effective mass of the bar to the mass, $M$, at $x=L$.

2. Elastic moduli. A sample of syntactic foam ${ }^{26}$ is cast into a solid rod of circular cross-section with a length of $14.96 \mathrm{~cm}$ and a diameter of $1.55 \mathrm{~cm}$. Its mass is $17.1 \mathrm{gm}$. The fundamental torsional and longitudinal resonances of the free-free vibrations of the rod were measured to be $4192 \mathrm{~Hz}$ and $6952 \mathrm{~Hz}$, respectively. Determine the shear modulus, Young's modulus, and Poisson's ratio of this syntactic foam sample.

3. M'bira. Also known as an African thumb piano, the m'bira, shown in Fig. 5.24, is an indigenous musical instrument of the Shona. The most common tuning is called Nyamaropa. It is similar to the western Mixolydian mode that is produced by playing a sequence of eight notes (no sharps or flats) starting and ending with "G" in an equally tempered scale (see Sect. 3.3.3).

Assuming that the width and thickness of the reeds are constant, and the vibrational frequencies are that of a clamped-free bar vibrating in its fundamental fixed-free mode, produce a table for the lengths of each reed, starting with $\mathrm{G}_{4}=392 \mathrm{~Hz}$ and ending with $\mathrm{G}_{5}=784 \mathrm{~Hz}$, if the reed for $\mathrm{G}_{5}$ is $5.0 \mathrm{~cm}$ long.

Fig. 5.24 African thumb piano

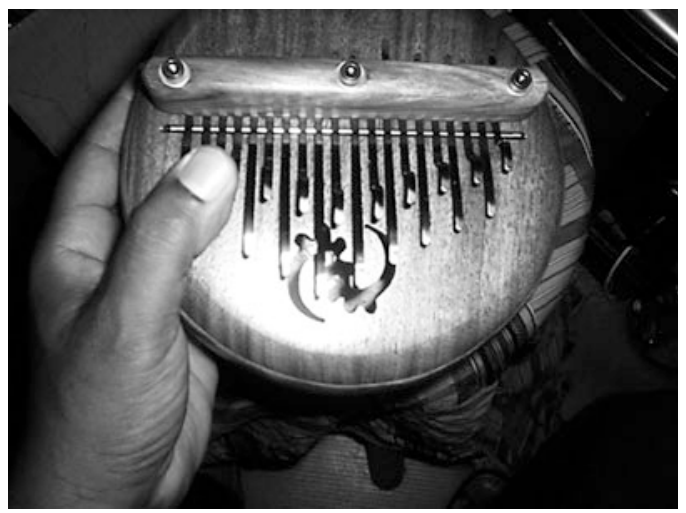

\footnotetext{
${ }^{26}$ Syntactic foam was developed in the 1960 s to provide buoyancy for instruments deployed in the deep ocean. They are composite materials fabricated by filling a castable epoxy with hollow glass microspheres. Those microspheres (sometimes also called microballoons) are very rigid, so the foam is not crushed when subjected to large hydrostatic pressures.
} 
Table 5.5 If you don't have easy access to the necessary items, you can use the frequencies listed at the right taken for a ruler with thickness, $t=0.79 \mathrm{~mm}$, and width, $w=24.0 \mathrm{~mm}$. Based on the length, $L=32.4 \mathrm{~cm}$, and the mass, $M=47.2 \mathrm{gm}$, the mass density of the ruler's material is $\rho=7680 \mathrm{~kg} / \mathrm{m}^{3}$

\begin{tabular}{l|l}
\hline Length $[\mathrm{m}]$ & Frequency $[\mathrm{Hz}]$ \\
\hline 0.29 & 7.09 \\
\hline 0.28 & 7.51 \\
\hline 0.25 & 9.53 \\
\hline 0.22 & 12.31 \\
\hline OJO & 14.96 \\
\hline 0.18 & 18.13 \\
\hline 0.16 & 22.97 \\
\hline 0.14 & 29.98 \\
\hline 0.12 & 40.67 \\
\hline
\end{tabular}

4. Modulus of a $\mathbf{1 2}^{\prime \prime}$ ruler. Try to determine the elastic modulus of a steel machinist's ruler by clamping it to your desk and measuring the frequency of vibration when you "twang" the ruler. (Machinists call that ruler a "scale.") Since the length of a ruler is already accurately marked, it will be easy to determine the vibrating length, although ensuring that the end attached to your desk is "clamped" may be more difficult. A C-clamp will probably provide an adequate restraint. The thickness of your ruler can be determined with a micrometer or Vernier calipers. Measuring the frequency of vibration may be more challenging, although many "smartphones" have an FFT frequency analyzer "app" available. Measure the vibration frequencies, $f_{n}$, as a function of the free length, $L_{n}$, of the ruler and use Eq. (5.50) to measure Young's modulus of your ruler. This technique has been used to determine Young's modulus of metallic glasses [13].

(a) Bar speed. Based on your data or the data in Table 5.5, think about how the data should be plotted and/or averaged to determine the bar speed, $c_{B}=[E / \rho]^{1 / 2}$, and its uncertainty if $|\delta t|$ $t \mid=0.6 \%$, corresponding to $\pm 1 \sigma$. Report your result and its relative uncertainty, providing any graphical output used to determine your result.

(b) Young's modulus. Use the ruler's mass density to determine the ruler's Young's modulus and its relative uncertainty. If you use the data in Table 5.5, you can let the relative uncertainty corresponding to $\pm 1 \sigma$ in the reported mass density to be $|\delta \rho / \rho|=1.0 \%$.

5. Complex modulus measurement. In Chap. 2, Problem 21 provided the amplitude and frequency at 22 points for the third torsional mode of a cylindrical rod, with a diameter of about $1 / 2 "=12.7 \mathrm{~mm}$, made from Eastman Tritan ${ }^{\circledR}$ Copolyester TX1001. The effective length of the rod for torsional vibration that included the mass loading due to the coils is $L_{\text {eff }}=31.94 \mathrm{~cm}$. The density of the rod is $\rho=1.18 \mathrm{gm} / \mathrm{cm}^{3}$.

(a) Normal mode frequency. ${ }^{27}$ The measured frequencies and amplitudes are provided in Table 2.2. Determine the resonance frequency, $f_{3}$, by choosing the largest measured amplitude and the two other amplitudes adjacent to the largest value. Fit those three frequency-amplitude pairs to a second-order polynomial, and use the resulting coefficients to make a quadratic interpolation to determine the best value for $f_{3}$. Plug $f_{3}$ back into that polynomial to determine the maximum amplitude of that third mode, $A_{3}(1)_{\max }$.

\footnotetext{
${ }^{27}$ If you have solved Prob. 21 in Chap. 2 by doing a least-squares fit of the data to the Rayleigh line shape of Eq. (2.63), you may use those results for $f_{3}, A_{3}(1)$, and $Q_{3}$ in parts (a) and (c) of this problem. Of course, if you just love to analyze high-quality resonance data (some of us do!), then do it both ways and compare your results. After that, make your friends and colleagues call you a "spectroscopist."
} 
(b) Shear modulus. Based on $L_{e f f}, \rho$, and $f_{3} / n$, determine the "real" part of the bar's shear modulus at $f_{3}$.

(c) Quality factor. Determine the two "down $3 \mathrm{~dB}$ " frequencies, $f_{+}$and $f_{-}$, by finding those frequencies which correspond to an amplitude of $A_{3}(1)_{\max } / \sqrt{2}$. Since none of the measured amplitudes exactly equal that $-3 \mathrm{~dB}$ amplitude, choose the two measurements that straddle $A_{3}(1)_{\max } / \sqrt{2}$ and fit a straight line between them to find $f_{-}$by linear interpolation. Do the same to find $f_{+}$. Use those two $-3 \mathrm{~dB}$ frequencies to calculate the quality factor, $Q_{3}$, for that $n=3$ torsional mode using Eq. (B.3).

(d) Loss modulus. Use Eq. (B.9) and your value for the elastic modulus you calculated in part (b) and $Q_{3}$ to determine the "imaginary" part of the complex shear modulus.

\section{References}

1. E. Mauceli et al., The Allegro gravitational wave detector: Data acquisition and analysis. Phys. Rev. D 54(2), 1264-1275 (1996)

2. M. McHugh et al., Calibration of the ALLEGRO resonant detector. Classical Quantum Gravity 22(18), S965-S973 (2005)

3. M. Janes, Sonic Pile Driving: The History and the Resurrection of Vibration Free Pile Driving. http://www. resonancetechnology.ca/res\%20driver\%20history\%20090112.pdf. A very impressive video comparing the resonant and drop hammer techniques is also available from that site: http://www.resonancetechnology.ca/gallery $\% 20 \mathrm{video} \%$ 201.php

4. G. Bodine, Jr., Sonic method and apparatus for driving casings through earthen formations. U.S. Patent No. 3,375,884 (April 2, 1968)

5. G. Bodine, Jr., Sonic method and apparatus for grinding rock material and the like to powder. U.S. Patent No. 3,429,512 (February 25, 1969)

6. S.P. Timoshenko, J.N. Goodier, Theory of Elasticity, 3rd edn. (McGraw-Hill, New York, 1970). See Chapter 11; ISBN 978-1-4020-7745-6

7. L.D. Landau, E.M. Lifshitz, Elasticity, 2nd edn. (Pergamon, New York, 1970).; See $§ 18$ and §25. ISBN 008006465 5

8. W.C. Young, R.G. Budynas, Roark's Formulas for Stress and Strain, 7th edn. (McGraw-Hill, New York, 2002); ISBN 0-07-072542-X

9. S.P. Timoshenko, History of Strength of Materials (McGraw-Hill, New York, 1953); ISBN 0-486-61187-6

10. Newton, Opticks or a Treatise on the Reflexions, Refractions, Inflections and Colours of Light, Also Two Treatises of the Species and Magnitude of Curvilinear Figures, 1st ed. (Smith \& Walford, 1704). 4th ed., with forward by A. Einstein and preface by I. B. Cohen (Dover, 1979); ISBN 978-0-486-60205-9

11. D. Heckerman, S. Garrett, G. Williams, P. Weidman, Surface tension restoring forces on gravity waves in a narrow channel. Phys. Fluids 22, 2270-2276 (1979)

12. S.M. Han, H. Benaroya, T. Wei, Dynamics of transversely vibrating beams using four engineering theories. J. Sound Vib. 225(5), 935-988 (1999)

13. M.B. Barmatz, H.J. Learny, H.S. Chen, A method for the determination of Young's modulus and internal friction in metallic glasses. Rev. Sci. Instrum. 79(6), 885-886 (1971)

14. R.P. Feynman, R.B. Leighton, M. Sands, The Feynman Lectures on Physics, vol II (Addison-Wesley, Reading, 1963), pp. 38-38

15. L.D. Landau, E.M. Lifshitz, Mechanics (Pergamon, Oxford, 1960). §39

16. N. Yazdi, F. Ayazi, K. Najafi, Micromachined inertial sensors. Proc. IEEE 86(8), 1640-1659 (1998)

17. W. Strutt (Lord Rayleigh), On waves propagated along the plane surface of an elastic solid. Proc. London Math. Soc. S1-17, 4-11 (1885)

18. F. Graff, Wave Motion in Elastic Solids (Clarendon, Oxford, 1975). Reprinted by Dover, 1991; ISBN 0-486-66745-6

19. S.L. Garrett, Resonant acoustic determination of elastic moduli. J. Acoust. Soc. Am. 88(1), 210-221 (1990)

20. E-Cast F-28 with Hardener 215, United Resins Corp., 1305 E. St. Gertrude Place, Unit B., Unit B, Santa Ana, CA 92705

21. A. Barone, A. Giacomini, Experiments on some electrodynamic ultrasonic vibrators. Acoustica 4, 182-184 (1954)

22. R.W. Leonard, Attenuation of torsional waves in Teflon. J. Acoust. Soc. Am. 40(1), 160-162 (1966) 
23. E.R. Fitzgerald, Simple method for observing audio frequency resonances and sound beams in crystals. J. Acoust. Soc. Am. 36(11), 2086-2089 (1964)

24. R.W. Leonard, Comments on the existence of the Fitzgerald effect. J. Acoust. Soc. Am. 38(4), 673-674 (1965)

25. Q.S. Guo, D.A. Brown, Determination of the dynamic elastic moduli and internal friction using thin resonant bars. J. Acoust. Soc. Am. 108(1), 167-174 (2000)

26. J. W. Strutt (Lord Rayleigh), Theory of Sound, 2nd edn. (Macmillan, London, 1894).; Reprinted by Dover, 1948, Vol. I, Ch. VII and Ch. VIII

27. Product Research \& Chemicals, Corp., 5454 San Fernando Road, Glendale, CA 91203

28. H. Tan, Resonant acoustic determination of complex elastic moduli (Unclassified), Master's Thesis in Engineering Acoustics, U. S. Naval Postgraduate School, Monterey, CA (March, 1991); DTIC Report No. AD A245 058

29. P. Horowitz, W. Hill, The Art of Electronics, 2nd edn. (Cambridge University Press, Cambridge, 1989). §15.15; ISBN 0-521-37095-7

30. R.E. Best, Phase-locked Loops: Design, Simulation, and Applications, 6th edn. (McGraw-Hill, New York, 2007); ISBN 978-0-07-149375-8

31. J.D. Ferry, Viscoelastic Properties of Polymers, 3rd edn. (Wiley, New York, 1980); ISBN: 978-0-471-04894-7

32. W.M. Visscher, A. Migliori, T.M. Bell, R.A. Reinert, On the normal modes of free vibration of inhomogeneous and anisotropic elastic objects. J. Acoust. Soc. Am. 90(4 Pt. 1), 2154-2162 (1991)

33. V.M. Keppens, J.D. Maynard, A. Migliori, Listening to materials: From auto safety to reducing the nuclear arsenal. Acoust. Today 6(2), 6-13 (2010)

34. P.S. Spoor, J.D. Maynard, A.R. Kortan, Elastic isotropy and anisotropy in quasicrystalline and cubic AlCuLi. Phys. Rev. Lett. 75(19), 3462-3465 (1995)

35. Y. Suzuki, V.R. Fanelli, J.B. Betts, F.J. Freibert, C.H. Mielke, J.N. Mitchell, M. Ramos, T.A. Saleh, A. Migliori, Temperature dependence of elastic moduli of polycrystalline $\beta$ plutonium. Phys. Rev. B 84, 064105 (2011)

36. P. S. Spoor, Elastic Properties of Novel Materials using PVDF Film and Resonant Ultrasound Spectroscopy, Ph. D. thesis in Acoustics, Penn State, December, 1996

37. P.M. Morse, Vibration and Sound (Acoustical Society of America, New York, 1976), pp. 166-170, §16. ISBN 0-88318-287-4

38. R.W. Young, Inharmonicity of plane wire piano strings. J. Acoust. Soc. Am. 24(3), 267-273 (1952)

Open Access This chapter is licensed under the terms of the Creative Commons Attribution 4.0 International License (http://creativecommons.org/licenses/by/4.0/), which permits use, sharing, adaptation, distribution and reproduction in any medium or format, as long as you give appropriate credit to the original author(s) and the source, provide a link to the Creative Commons license and indicate if changes were made.

The images or other third party material in this chapter are included in the chapter's Creative Commons license, unless indicated otherwise in a credit line to the material. If material is not included in the chapter's Creative Commons license and your intended use is not permitted by statutory regulation or exceeds the permitted use, you will need to obtain permission directly from the copyright holder. 